\title{
Nevada Test Site \\ Environmental Report 2004
}

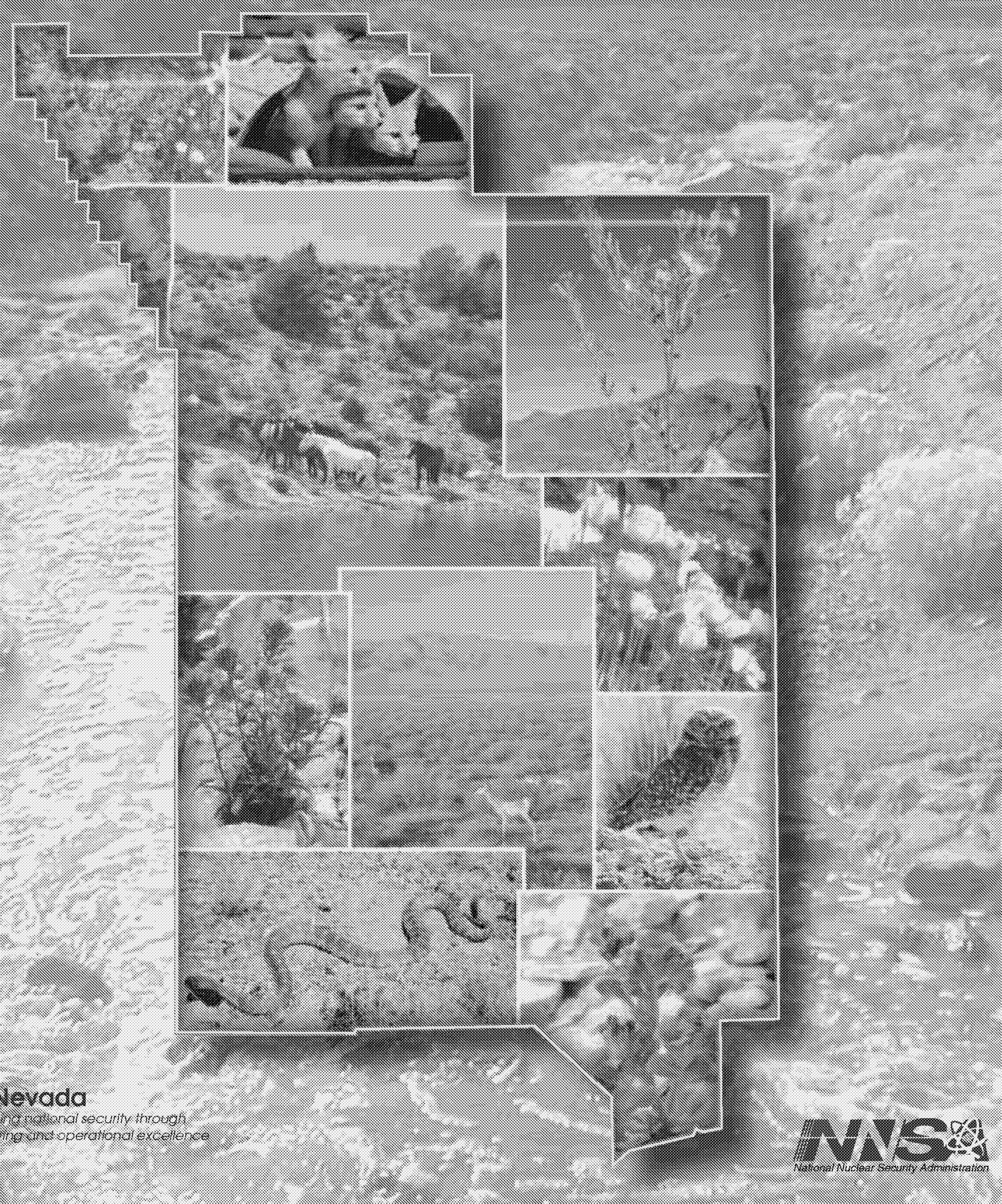




\title{
Disclaimer
}

Reference herein to any specific commercial product, process, or service by trade name, trademark, manufacturer, or otherwise, does not necessarily constitute or imply its endorsement, recommendation, or favoring by the United States Government or any agency thereof or its contractors or subcontractors.

Available for sale to the public from:

\author{
U.S. Department of Commerce \\ National Technical Information Service \\ 5285 Port Royal Road \\ Springfield, VA 22161-0002 \\ Telephone: (800) 553-6847 \\ Fax: (703) 605-6900 \\ E-mail: orders@ntis.gov \\ Online ordering: htrp:/wwww.ntis.gov/ordering.hm
}

Available electronically at httr:/ www ostigov/bridge

Available for a processing fee to the U.S. Department of Energy and its contractors, in paper, from:

U.S. Department of Energy

Office of Scientific and Technical Information

P.O. Box 62

Oak Ridge, TN 37831-0062

Telephone: (865) 576-8401

Fax: (865) 576-5728

E-mail: reports@adonis.osti.gor 


\title{
Nevada Test Site Environmental Report 2004
}

\author{
Senior Author: Cathy A. Wills \\ Technical Editor: Angela L. McCurdy
}

October 2005

Work Performed Under

Contract No. DE-AC08-96NV11718

\author{
Prepared for: \\ U. S. Department of Energy \\ National Nuclear Security Administration \\ Nevada Site Office
}

Prepared by:

Bechtel Nevada

Post Office Box 98521

Las Vegas, NV 89193-8521 
THIS PAGE INTENTIONALLY LEFT BLANK 


\section{Acknowledgements}

Environmental Technical Services (ETS) of Bechtel Nevada (BN) is responsible for production of this document. Environmental monitoring and compliance data were gathered through the combined efforts of several BN organizations in addition to ETS: Environmental Services, Radioactive Waste Operations, Solid Waste Operations, Environmental Restoration, Ecological Services, Radiological Control, Occupational Safety \& Health, and Contractor Assurance \& Compliance. The Water Resources and Cultural Resources divisions of the Desert Research Institute (DRI) contributed data for offsite water and air monitoring and cultural resource protection. My sincere thanks go to each of the authors and contributors from these organizations that are listed below. Your generous help and perseverance during the writing and review phases were greatly appreciated.

\section{Chapter Authors and Contributors}

\section{Executive Summary}

Cathy A. Wills, BN

\subsection{Introduction}

Robert F. Grossman, BN

Cathy A. Wills, BN

\subsection{Compliance Summary}

Colleen M. Beck, DRI

Shaughn A. Burnison, BN

Elizabeth C. Calman, BN

Douglas K. Clark, BN

Andrea L. Gile, BN

Cirilo C. Gonzales, BN

Dennis L. Gustafson, BN

Robert F. Grossman, BN

Madelyn A. Hayes, BN

Orin L. Haworth, BN

Alfred J. Karnes, BN

Coby Moke, BN

Teresa M. Morgan, BN

Paul K. Ortego, BN

Phyllis M. Radack, BN

Stuart E. Rawlinson, BN

Carlton Soong, BN

Tammy H. Wallace, BN

Karen E. Williams, BN

\subsection{Radiological and Non-Radiological} Air Monitoring

Elizabeth C. Calman, BN

Charles B. Davis, EnviroStat

Robert F. Grossman, BN

Teresa M. Morgan, BN

Tammy H. Wallace, BN

Phillip D. Worley, BN

\subsection{Radiological and Non-Radiological} Water Monitoring

David B. Hudson, BN

Coby Moke, BN

Paul K. Ortego, BN

Phyllis M. Radack, BN

Ronald W. Warren, BN

\subsection{Direct Radiation Monitoring}

Ronald W. Warren, BN

\subsection{Oversight Radiological Monitoring}

of Air and Water

William T. Hartwell, DRI

Craig Shadel, DRI

Charles E. Russell, DRI

\subsection{Radiological Biota Monitoring}

Ronald W. Warren, BN

\subsection{Radiological Dose Assessment}

Robert F. Grossman, BN

Ronald W. Warren, BN

\subsection{Waste Management and}

\section{Environmental Restoration}

Shaughn A. Burnison, BN

Douglas K. Clark, BN

Cirilo C. Gonzales, BN

Michelle A. Hallmark, BN

Gary M. Romano, Stoller-Navarro

Carlton Soong, BN

Karen E. Williams, BN 
10.0 Hazardous Materials Control and

Management

Orin L. Haworth, BN

Madelyn A. Hayes, BN

Coby P. Moke, BN

\subsection{Pollution Prevention}

Alfred J. Karnes, BN

12.0 Historic Preservation and Cultural

\section{Resources Management}

Colleen M. Beck, DRI

Barbara A. Holz, DRI

Robert C. Jones, DRI

\subsection{Ecological Monitoring}

David C. Anderson, BN

Paul D. Greger, BN

Derek B. Hall, BN

Dennis J. Hansen, BN

W. Kent Ostler, BN

Cathy A. Wills, BN

\subsection{Underground Test Area Project}

Sigmund L. Drellack, BN

15.0 Hydrologic Resources Management

Program

Bruce W. Hurley, NNSA/NSO

16.0 Meteorological Monitoring

Darryl Randerson, ARL-SORD
17.0 Environmental Management System

Orin L. Haworth, BN

18.0 Compliance Quality Assurance Program

Jerry J. Dugas, BN

Theodore J. Redding, BN

19.0 Oversight Quality Assurance Program

Craig Shadel, DRI

Appendix A: NTS Description

Sigmund L. Drellack, BN

Harold Drollinger, DRI

Robert F. Grossman, BN

Darryl Randerson, ARL-SORD

Cathy A. Wills, BN

Appendix B: NTS Satellite Facilities

Elizabeth C. Calman, BN

Sigmund L. Drellack, BN

Madelyn A. Hayes, BN

Coby P. Moke, BN

Ronald W. Warren, BN

Appendix C: Helpful Information

Modified from Hanford Site 1997

Environmental Report

Charles B. Davis, EnviroStat

Appendix D: Glossary

Modified from Lawrence Livermore

National Laboratory 2003

Enivionmental Report

Appendix E: Acronyms and Abbreviations

Cathy A. Wills, BN

\section{ETS Support Staff}

The following individuals within ETS are responsible year-in and year-out for the numerous tasks that are integral to the collection, quality assurance, and quality control of much of the environmental data reported in this NTSER.

These are the "behind the scenes" folks. Thank you all for your efforts in the following areas.

Administration of the Bechtel Environmental

Integrated Data Management System

(BEIDMS) for Routine Radiological

Environmental Monitoring Program (RREMP), Clean Water Act (CWA) and Safe Drinking

Water Act (SDWA) Projects:

Elizabeth Burns

Robert G. Peppard
Coordination of Field Sampling Operations for RREMP, CWA, and SDWA Projects:

Terrance P. Sonnenburg

Field Sampling for RREMP, CWA, and SDWA Projects:

Martin D. Cavanaugh

Paul D. Greger

David D. Rudolph

Matthew $\mathrm{O}$. Weaver

Alan O. Wittig 
Laboratory Operations Supporting RREMP Screening and Sample Processing:

Lynn N. Jaussi

Sample Management Supporting the BN Subcontracting of Environmental Analytical Services:

Catherine D. Castaneda

Theodore J. Redding
RREMP Data Verification, Validation, and

Review:

Elizabeth Burns

Robert F. Grossman

David B. Hudson

Ronald W. Warren

Quality Assurance Oversight of the RREMP: Jerry J. Dugas

\section{Report Production and Distribution Support Personnel}

Lastly, my appreciation goes to the following individuals who were responsible for improving the quality, appearance, and timely production and distribution of this report:

Charles Davis of EnviroStat provided support for the statistical analyses and interpretation of NTS radiological monitoring data collected by ETS. Charles also collaborated in editing the document for clarity and accuracy of the text and data presentation.

Bob Arnold of BN Technical Publications and Graphics Services (TPGS) designed the cover for this document and the 2004 Nevada Test Site Environmental Report Summary (NTSER Summary). Elaine Upson of BN TPGS designed the layout of the NTSER Summary.

Tom Fitzmaurice of BN Environmental Restoration provided his artistic talents to produce the drawing of possible pathways of radiological exposure to the public presented in the NTSER Summary.

Ashley Cushman of BN Geographic Information Systems (GIS)/Data Management is a GIS specialist who worked with all the authors to produce the high-quality GIS-generated maps and figures.

Kurt Jahn and Mark Shaw of Office Services are thanked for producing high-quality hardcopies and compact discs of the NTSER and the NTSER Summary under a tight production schedule.

Fina Martinez-Myers of Office Services oversaw the Government Printing Office subcontract, the print production schedule, the acquisition of all printing and mailing supplies, and the timely distribution of all of the NTSER products. Thank you for your impeccable customer service.

A very special thank you is reserved for Angela McCurdy of BN TPGS. She is the word processing specialist responsible for the final layout and format of the NTSER. Her skill and dedication applied to this project year after year are acknowledged. Thank you, Angela. 
THIS PAGE INTENTIONALLY LEFT BLANK 


\section{Table of Contents}

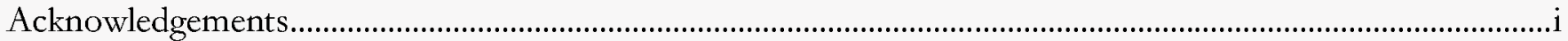

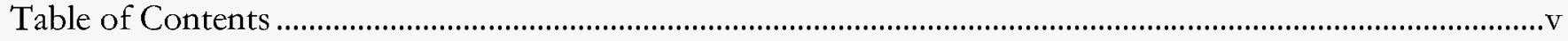

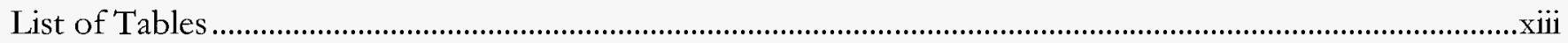

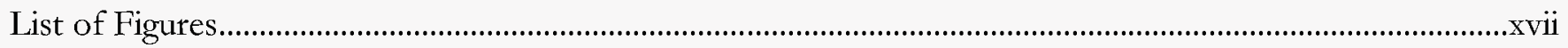

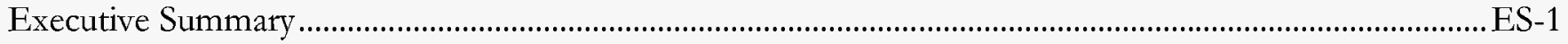

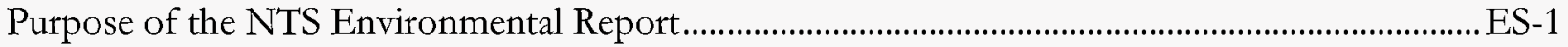

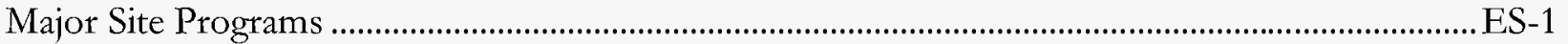

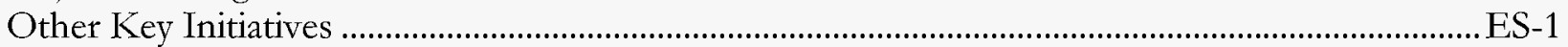

Environmental Performance Measure Programs ..............................................................................ES-2

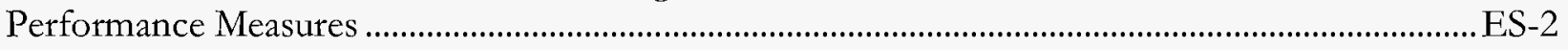

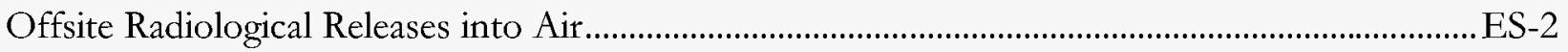

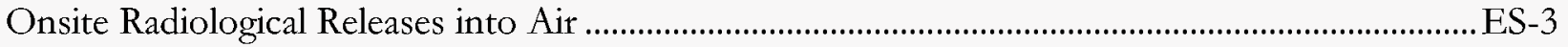

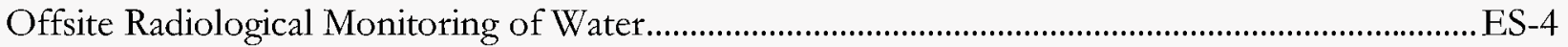

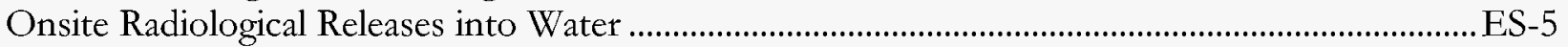

Estimated Radiation Dose to the Public.................................................................................................

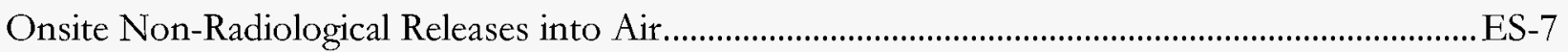

Onsite Non-Radiological Releases into Water............................................................................................

Onsite Non-Radiological Drinking Water Quality....................................................................................

Non-Radiological Releases into Air and Water at the NLVF..................................................................

Accidental or Unplanned Environmental Releases or Occurrences ……............................................. ES-9

Pollution Prevention/Waste Minimization Activities ..........................................................................

Overall Compliance with Environmental Laws, Regulations, and Policies ...................................... ES-9

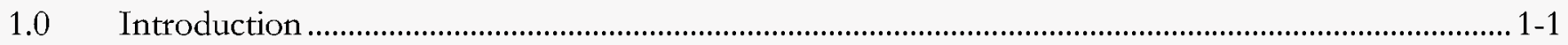

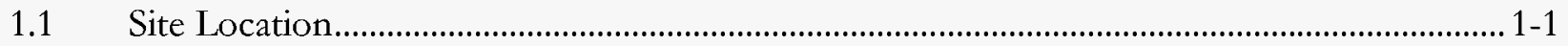

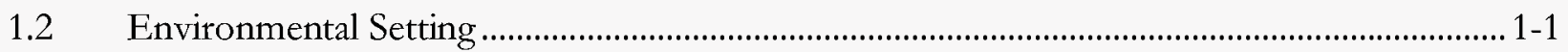

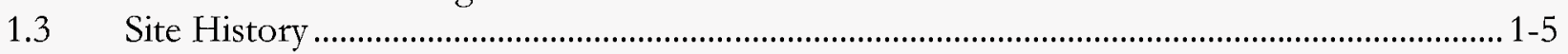

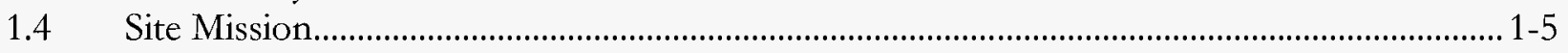

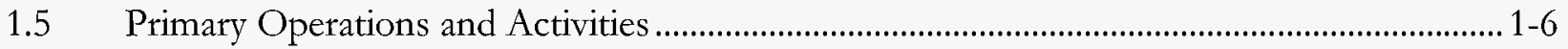

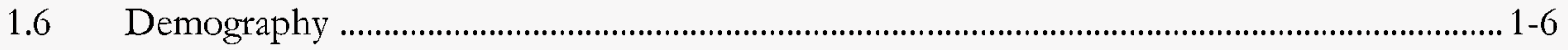

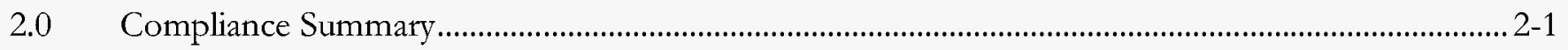

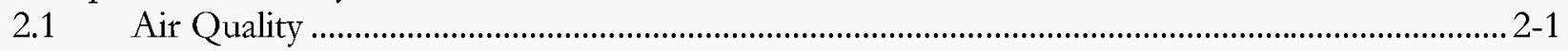

Clean Air Act (CAA), National Emission Standards for Hazardous Air Pollutants

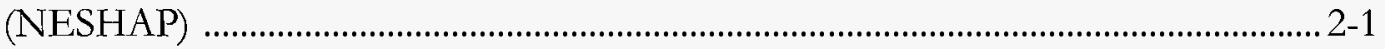

CAA, National Ambient Air Quality Standards (NAAQS)................................................... 2-1

CAA, New Source Performance Standards (NSPS) ........................................................... 2-2

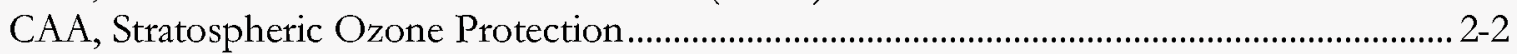

Other NTS Air Quality Permit Requirements..................................................................2 2-2

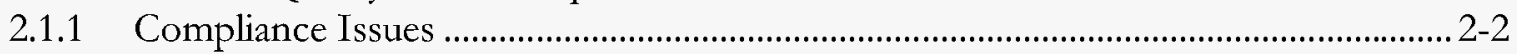

2.1.2 Compliance Reports .................................................................................................. 2-3

2.1.3 Compliance Status........................................................................................................ 2-3

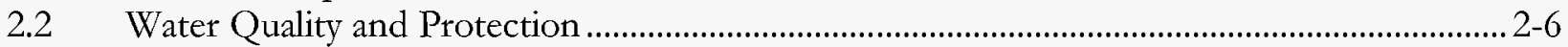

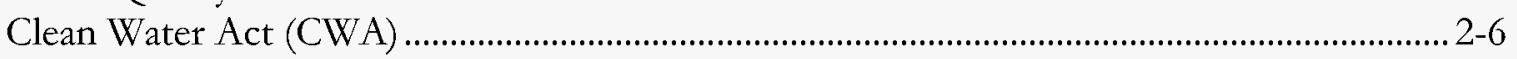

Safe Drinking Water Act (SDWA) ................................................................................. 2-6

Nevada Administrative Code (NAC) 445A - Water Controls (Public Water Systems) ......... 2-6 
NAC 444 and 445A - Water Controls (Water Pollution Control)

NAC 534 - Nevada Division of Water Resources Regulations for Water Well and Related Drilling

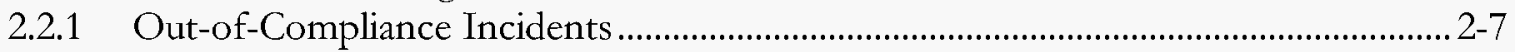

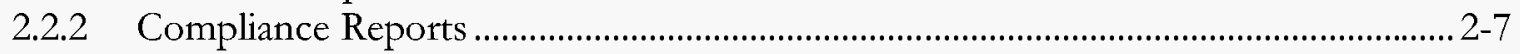

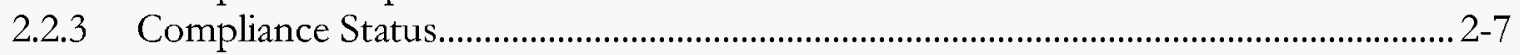

2.3 Radiation Dose Protection ............................................................................................... 2-10

Clean Air Act (CAA), National Emission Standards for Hazardous Air Pollutants

(NESHAP)

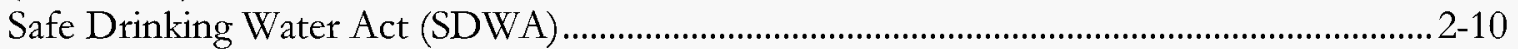

DOE Order 5400.5 Radiation Protection of the Public and the Environment .................................. 2-10

DOE Standard DOE-STD-1153-2002 …..................................................................................... 2-10

DOE Order 435.1 Radioactive $W$ aste Management......................................................................2-10

DOE Order 450.1 Environmental Protection Program................................................................2-10

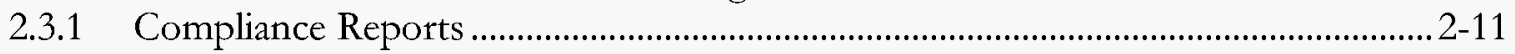

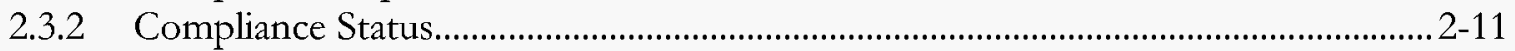

2.4 Radioactive and Non-Radioactive Waste Management and Environmental

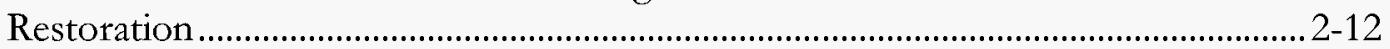

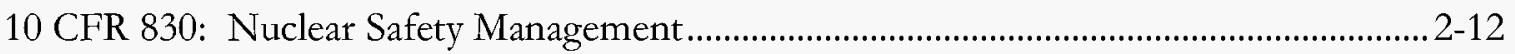

DOE Order 435.1 Radioactive $W$ aste Management....................................................................... 2-12

Atomic Energy Act (AFA) .................................................................................................... 2-12

Resource Conservation and Recovery Act (RCRA) .......................................................... 2-12

Comprehensive Environmental Response, Compensation, and Liability Act

(CERCLA) / Superfund Amendments and Reauthorization Act (SARA) ...............2-13

Federal Facility Compliance Act (FFCA) ........................................................................2-13

Federal Facilities Agreement and Consent Order (FFACO) ................................................. 2-13

40 CFR Subchapter I, Parts 239-299: Solid Wastes...................................................................... 2-13

NAC 444.570-7499 - Solid Waste Disposal....................................................................... 2-13

2.4.1 Compliance Reports .................................................................................................. 2-13

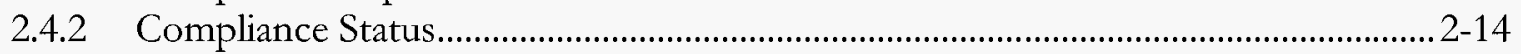

2.5 Hazardous Materials Control and Management ................................................................. 2-17

Toxic Substances Control Act (TSCA) ............................................................................... 2-17

Federal Insecticide, Fungicide, and Rodenticide Act (FIFRA) ............................................ 2-17

Emergency Planning and Community Right-to-Know Act (EPCRA) .................................. 2-17

NAC Chapter 555 - Control of Insects, Pests, and Noxious Weeds ..................................... 2-17

NAC Chapter 444 - Polychlorinated Biphenyls ....................................................................... 2-18

State of Nevada Chemical Catastrophe Prevention Act......................................................... 2-18

2.5.1 Compliance Reports ................................................................................................ 2-18

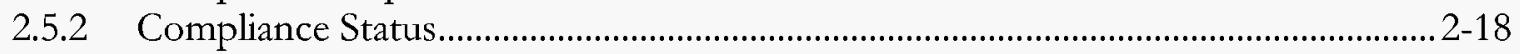

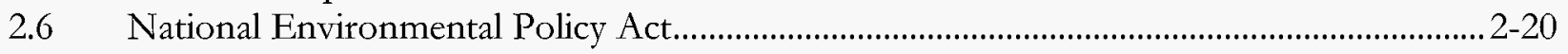

2.7 Pollution Prevention and Waste Minimization ................................................................... 2-22

Resource Conservation and Recovery Act of 1976 (RCRA) .................................................2-22

EO 13101 Greening the Government through W aste Prevention, Recycling and

Federal Acquisition .......................................................................................................... 2-22

DOE Order 450.1 Environmental Protection Program.................................................................22-22

NDEP Hazardous Waste Permit Number NEV HW009......................................................... 2-22

Secretary of Energy's Pollution Prevention and Energy Efficiency Leadership Goals........ 2-22

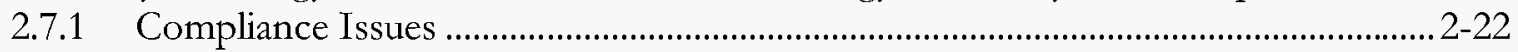

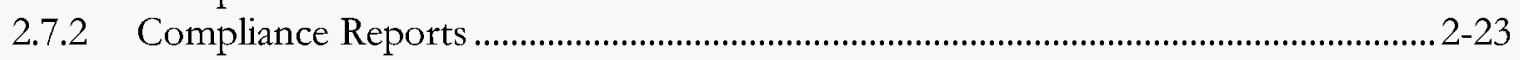


2.7.3 Compliance Status..................................................................................................... 2-23

2.8 Historic Preservation and Cultural Resource Protection........................................................... 2-25

National Historic Preservation Act of 1966 ........................................................................... 2-25

EO 11593 Protection and Enhancement of the Cultural Environment ...............................................2-25

Archeological Resources and Protection Act of 1979.............................................................. 2-25

American Indian Religious Freedom Act of 1978 …………………………………….....2-25

Native American Graves Protection and Repatriation Act of 1990 (NAGPRA) .................2-25

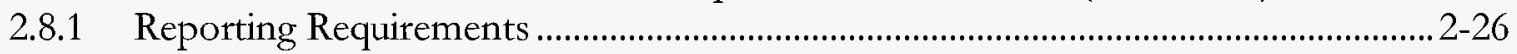

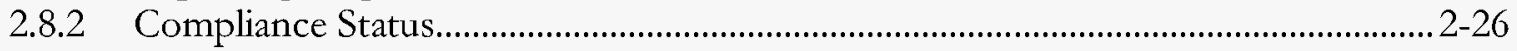

2.9 Conservation and Protection of Biota and Wildlife Habitat.................................................. 2-28

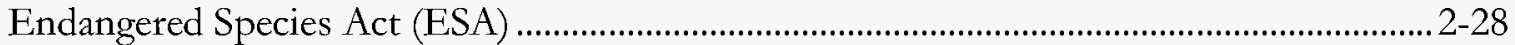

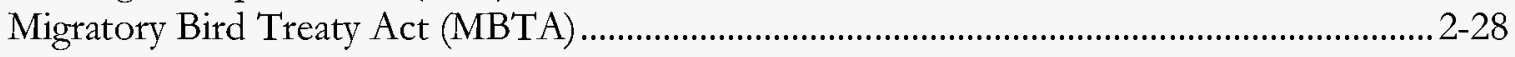

Bald Eagle Protection Act................................................................................................ 2-28

Clean Water Act, Section 404, Wetlands Regulations ............................................................ 2-28

National Wildlife Refuge Administration Act.......................................................................2-2-28

EO 11990 Protection of Wetlands ......................................................................................... 2-28

EO 11988 Floodplain Management ....................................................................................2-28

EO 13186 Responsibilities of Federal Agencies to Protect Migratory Birds .........................................2-28

EO 13112 Invasive Species ................................................................................................... 2-28

Wild Free-Roaming Horse and Burro Act................................................................................2-29

Five-Party Cooperative Agreement.........................................................................................2-29

NAC 503.010 - 503.104 - Protection of Wildlife .................................................................. 2-29

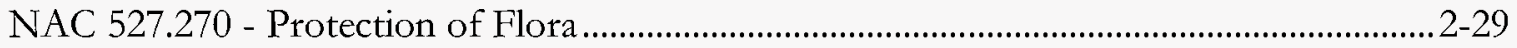

2.9.1 Out-of-Compliance Incidents......................................................................................2-29

2.9.2 Compliance Reports ............................................................................................. 2-29

2.9.3 Compliance Status....................................................................................................... 2-29

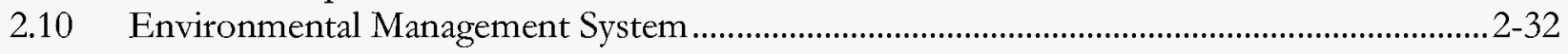

EO 13148 Greening the Government through Leadership in Environmental Management ...................2-32

DOE Order 450.1 Environmental Protection Program..................................................................2-32

2.10 .1 Compliance Reports ................................................................................................ 2-32

2.10.2 Compliance Status.................................................................................................. 2-32

2.11 Occurrences, Unplanned Releases, and Continuous Releases ..................................................2-33

Comprehensive Environmental Response, Compensation, and Liability

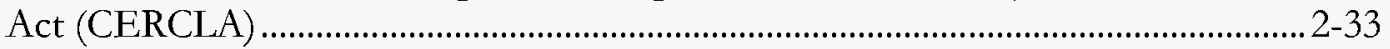

Emergency Planning and Community Right-to-Know Act (EPCRA)................................... 2-33

40 CFR 302.1 - 302.8: Designation, Reportable Quantities, and Notification ......................2-33

DOE Order 231.1A Emvironment, Safety, and Health Reporting...................................................2-33

NAC 445A.345-445.348 - Notification of Release of Pollutant ..............................................2-33

Water Pollution Control General Permit GNEV93001 ……………………………………....2-233

Other NTS Permits/Agreements............................................................................................. 2-33

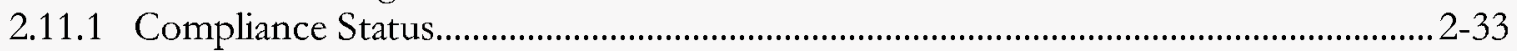

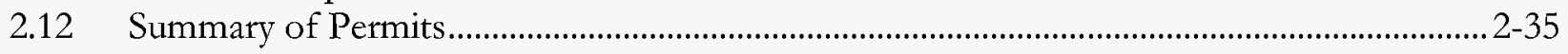

3.0 Radiological and Non-Radiological Air Monitoring .............................................................................. 3-1

$3.1 \quad$ Radiological Air Monitoring............................................................................................. 3-1

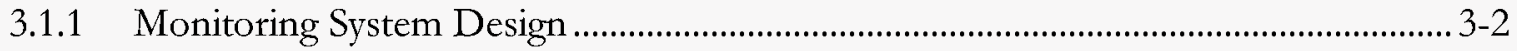

3.1.2 Air Particulate and Tritium Sampling Methods............................................................ 3-4

3.1.3 Presentation of Air Sampling Data............................................................................... 3-5

3.1.4 Air Sampling Results from Environmental Samplers.................................................... 3-5 


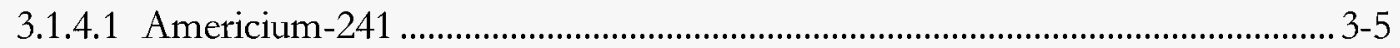

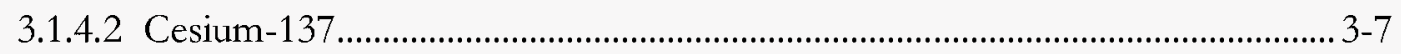

3.1.4.3 Plutonium Isotopes........................................................................................... $3-7$

3.1.4.4 Uranium Isotopes................................................................................... $3-12$

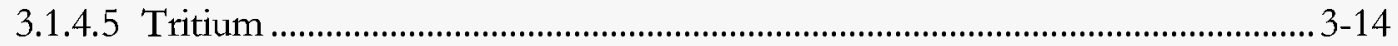

3.1.4.6 Gross Alpha and Gross Beta..................................................................... 3-16

3.1.5 Air Sampling Results from Critical Receptor Samplers............................................... 3-20

3.1.6 Air Sampling Results from Point-Source (Stack) Sampler.......................................... 3-20

3.1.7 Radiological Atmospheric Releases used for Estimating Dose to the Maximally

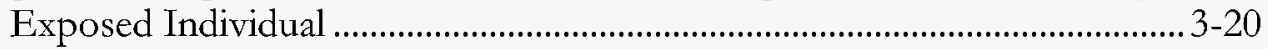

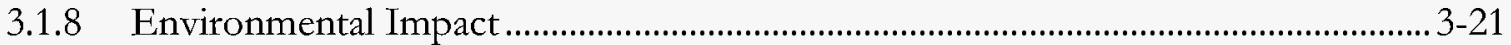

3.2 Non-Radiological Air Quality Assessment ………………………………………................ 3-22

3.2.1 Emissions of Criteria Air Pollutants and Hazardous Air Pollutants .......................... 3-23

3.2.2 Production Rates/Hours of Operation ……………………………………………..... 3-26

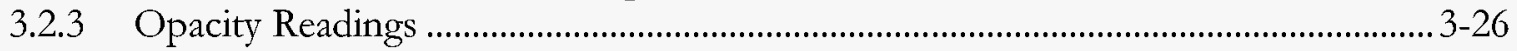

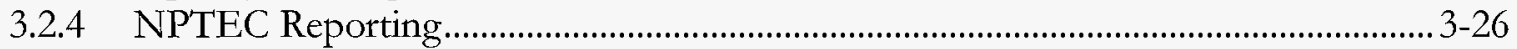

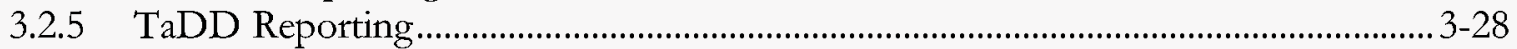

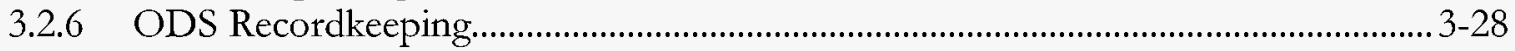

3.2.7 Asbestos Abatement................................................................................................ 3-28

3.2.8 Fugitive Dust Control ………………………………………………………...... 3-29

3.2.9 State Inspections ……………………………………………………………… 3-29

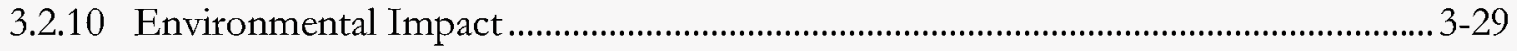

4.0 Radiological and Non-Radiological Water Monitoring ........................................................................ 4-1

4.1 Radiological Surface Water and Groundwater Monitoring........................................................ 4-1

4.1.1 Water Monitoring Locations .............................................................................................. 4-3

4.1.2 Water Sampling/Analysis Methods................................................................................ 4-4

4.1.3 Presentation of Water Sampling Data...……………………………………………….... 4-7

4.1.4 Results from Offsite Wells .......................................................................................... 4-7

4.1.5 Results from Offsite Springs .................................................................................. 4-9

4.1.6 Results from NTS Potable Water Supply Wells ........................................................... 4-11

4.1.7 Results from NTS Monitoring Wells ........................................................................... 4-14

4.1.8 Results from NTS E Tunnel Ponds ....................................................................... 4-16

4.1.9 Results from NTS Sewage Lagoons.............................................................................. 4-18

4.1.10 UGTA Wells .......................................................................................................... 4-19

4.1.11 Environmental Impact ....................................................................................... 4-21

4.2 Non-Radiological Drinking Water and Wastewater Monitoring............................................ 4-22

4.2.1 Drinking Water Monitoring........................................................................................ 4-22

4.2.1.1 Water Quality of PWS and Permitted Water Hauling Trucks.......................4-22

4.2.1.2 Sanitary Survey of PWS and Inspection of Permitted Water

Hauling Trucks .................................................................................................. 4-26

4.2.2 Domestic Wastewater Monitoring............................................................................. 4-27

4.2.3 Industrial Wastewater Monitoring................................................................................. 4-27

4.2.3.1 Quarterly Analysis of Influent Water Quality ................................................ 4-29

4.2.3.2 Annual Analysis of Toxicity of Sewage Lagoon Pond Waters ...................... 4-29

4.2.3.3 Annual Analysis of Groundwater Monitoring Wells Associated

With Sewage Lagoons....................................................................................... 4-30

4.2.3.4 Sewage System Inspections............................................................................... 4-31 


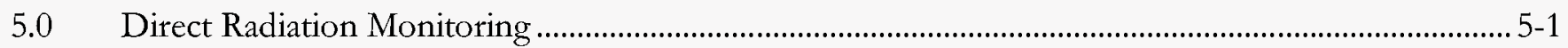

$5.1 \quad$ Measurement of Direct Radiation ............................................................................................. 5-1

5.2 TLD Surveillance Network Design......................................................................................... 5-2

5.2.1 Data Quality ………………………………………………………………….... 5-2

5.2.2 Data Reporting …………………………………………………………………. 5-4

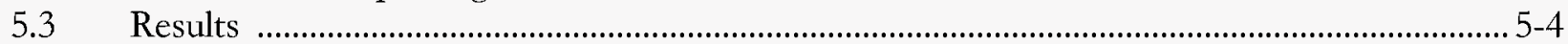

5.3.1 Potential Exposure to the Public along the NTS Boundary......................................... 5-7

5.3.2 Exposure Rates at Radioactive Waste Management Sites (RWMSs) ............................ 5-8

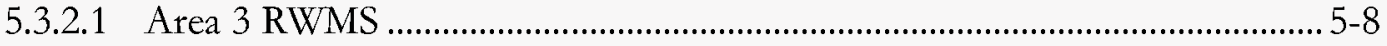

5.3.2.2 Area 5 RWMS .................................................................................. 5-8

5.3.3 Exposure Rates from NTS Operational Activities ....................................................... 5-9

5.3.4 Exposure to NTS Plants and Animals ........................................................................... 5-10

5.3.5 Exposure Rate Patterns in the Environment over Time …………………………..... 5-10

$5.4 \quad$ Environmental Impact........................................................................................................... 5-11

6.0 Oversight Radiological Monitoring of Air and Water ............................................................................. 6-1

6.1 Offsite Air Monitoring.............................................................................................................. 6-1

6.1.1 Air Particulate Sampling Results.................................................................................... 6-4

6.1.1.1 Gross Alpha and Gross Beta.............................................................................. 6-4

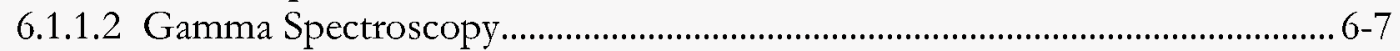

6.1.2 TLD Results........................................................................................................ 6-7

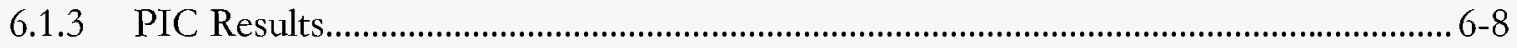

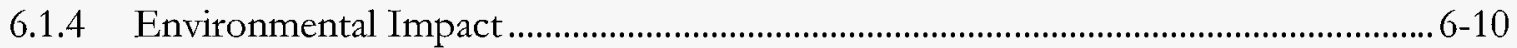

6.2 Offsite Surface and Groundwater Monitoring......................................................................... 6-11

6.2.1 Sample Locations and Methods..................................................................................6-11

6.2.2 Procedures and Quality Assurance.................................................................................. 6-11

6.2.3 Results of Surface Water Monitoring from Springs...................................................... 6-14

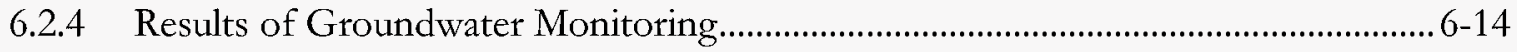

6.2.5 Environmental Impact ............................................................................................. 6-14

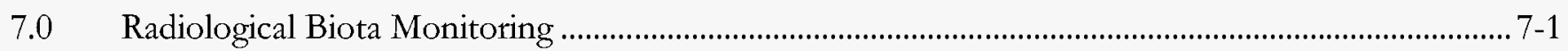

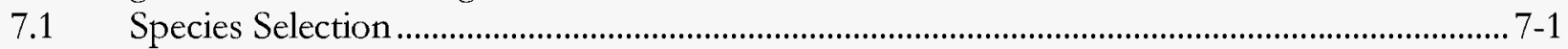

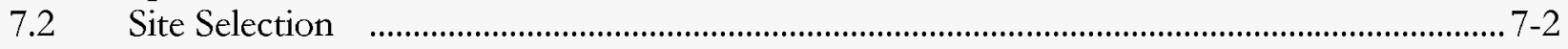

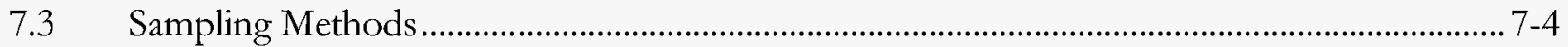

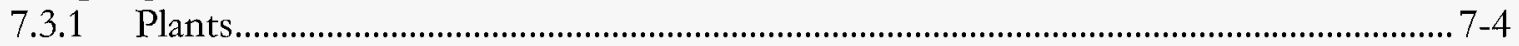

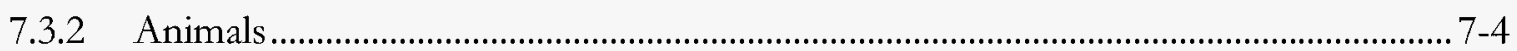

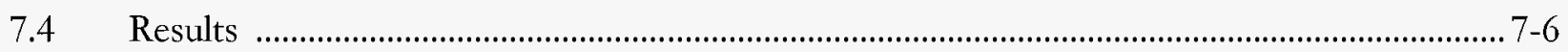

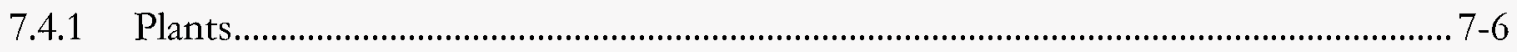

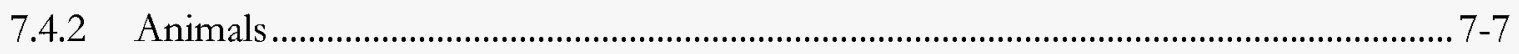

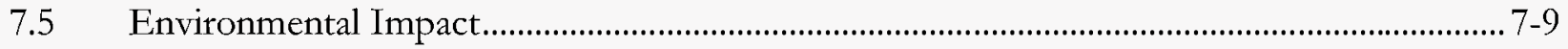

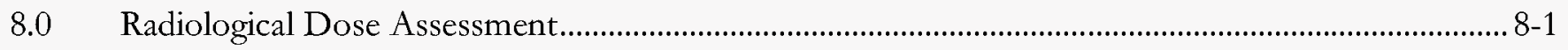

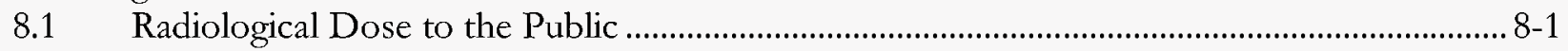

8.1.1 Determining Human Exposure Pathways ...................................................................... 8-1

8.1.2 Identifying Onsite Sources and Radionuclide Air Emission Rates............................... 8-2

8.1.3 Calculating Dose to Humans from NTS Air Emissions................................................ 8-2

8.1.4 Calculating Dose to Humans from Ingestion of Wild Game from the NTS ............ 8-5

8.1.5 Estimating Dose to the Public from Release of Property Containing

Residual Radioactive Material....................................................................................... 8-7 
8.1.6 Total Offsite Dose to the Maximally Exposed Individual (MEI)............................... 8-8

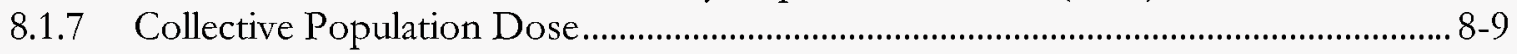

$8.2 \quad$ Dose to Aquatic and Terrestrial Biota .................................................................................... 8-10

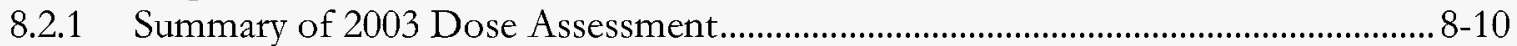

8.2.2 New Radionuclide Sources Accessible to Biota in 2004 .......................................... 8-13

8.2.3 2004 Site-Specific Biota Dose Assessment ..................................................................... 8-13

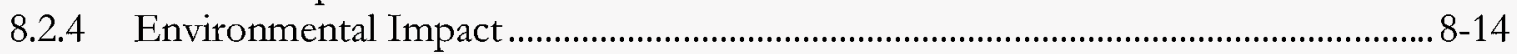

$9.0 \quad$ Waste Management and Environmental Restoration ................................................................... 9-1

9.1 Radioactive Waste Management............................................................................................. 9-1

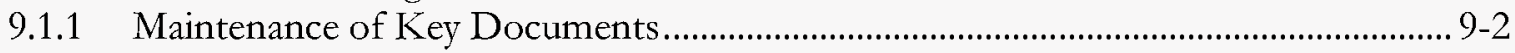

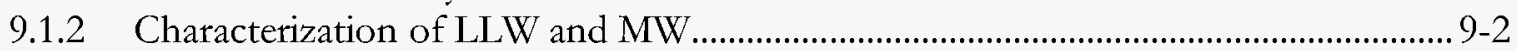

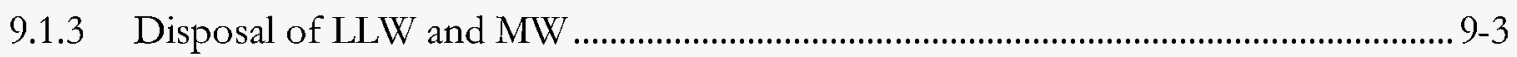

9.1.4 TRU Waste Operations.................................................................................................. 9-4

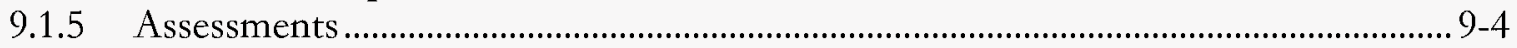

9.1.6 Groundwater Monitoring for LLW Pit P03U ............................................................

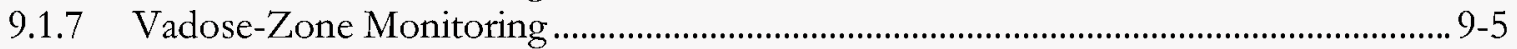

9.1.7.1 Area 3 RWMS Drainage Lysimeter Facility ................................................. 9-5

9.1.7.2 Area 5 RWMS Weighing Lysimeter Facility .................................................. 9-5

9.1.7.3 Area 5 RWMS Automated Monitoring.......................................................... 9-6

9.1.7.4 RWMS Supplemental Automated Monitoring................................................... 9-6

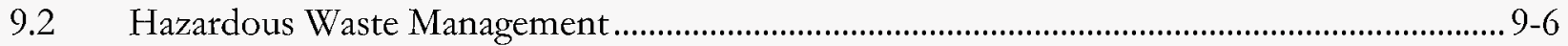

9.3 Underground Storage Tank (USTs) Management .............................................................. 9-7

9.4 Environmental Restoration - Remediation of Historic Contaminated Sites .......................... 9-7

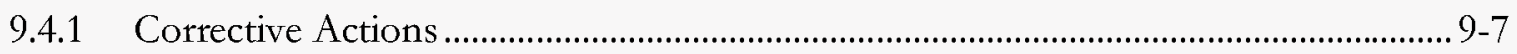

9.4.2 Post-Closure Monitoring and Inspections ................................................................... 9-9

9.5 Solid and Sanitary Waste Management..................................................................................... 9-12

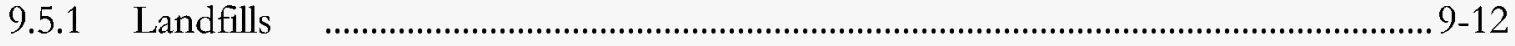

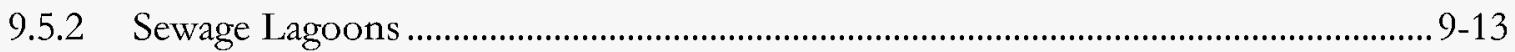

10.0 Hazardous Materials Control and Management ....................................................................... 10-1

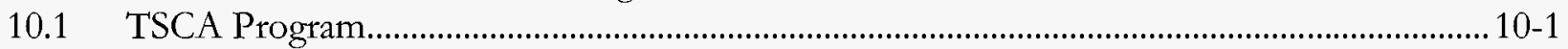

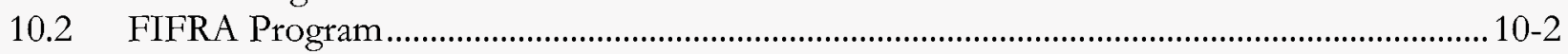

10.3 EPCRA Program ....................................................................................................... 10-2

10.4 Nevada Chemical Catastrophe Prevention Act.................................................................. 10-3

11.0 Pollution Prevention and Waste Minimization............................................................................. 11-1

11.1 P2 and WM Goals and Components..........................................................................11-1

11.2 Major P2/WM Accomplishments in 2004........................................................................11-2

11.3 Waste Generation in 2004 Compared To Prior Years ........................................................11-2

11.4 Waste Reductions in 2004 Compared To Prior Years ............................................................11-3

11.5 Secretary of Energy's P2/WM Leadership Goals..................................................................... 11-4

12.0 Historic Preservation and Cultural Resources Management............................................................. 12-1

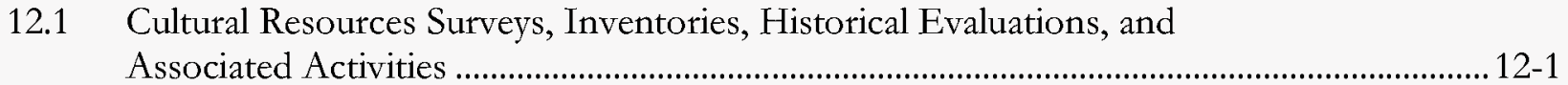

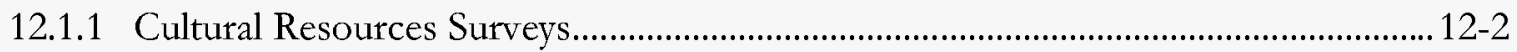

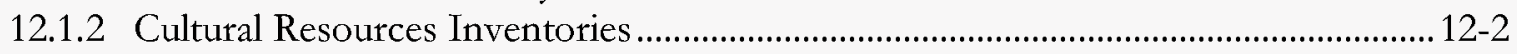

12.1.3 Evaluations of Historic Structures ...................................................................... 12-2 
12.1.4 Associated Cultural Resources Activities................................................................12-3

12.1.4.1 Adverse Effect Assessments and Mitigation Activities ..............................12-3

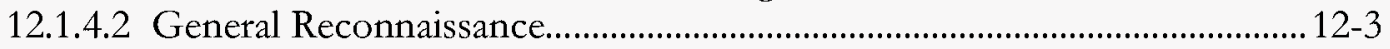

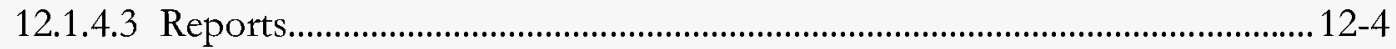

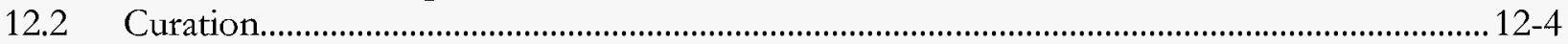

12.3 American Indian Program ........................................................................................... 12-5

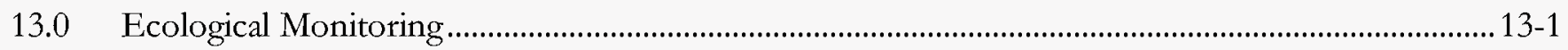

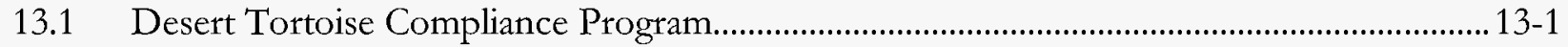

13.2 Biological Surveys at Proposed Project Sites.....................................................................13-3

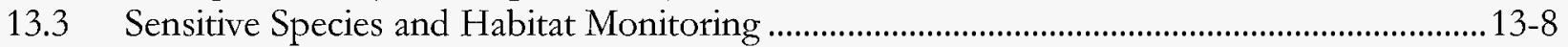

13.3.1 Sensitive Plants .......................................................................................................13-9

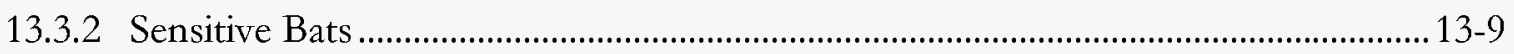

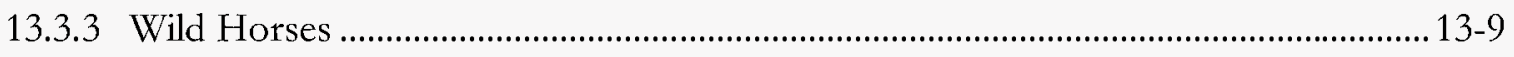

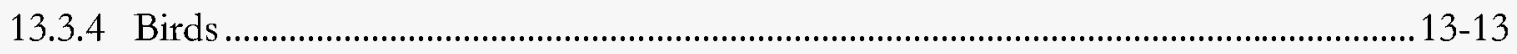

13.3.5 Natural and Human-Made Water Sources .............................................................. 13-15

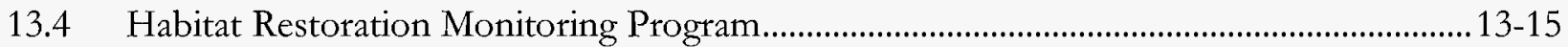

13.5 Biological Monitoring of the NPTEC ..............................................................................13-16

$14.0 \quad$ Underground Test Area Project............................................................................................... 14-1

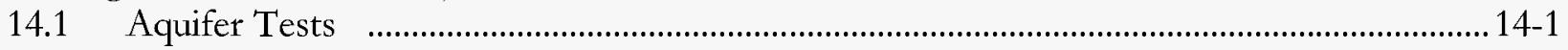

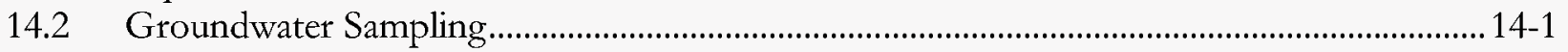

14.3 3D Hydrostratigraphic Framework Models................................................................... 14-1

$15.0 \quad$ Hydrologic Resources Management Program ............................................................................ 15-1

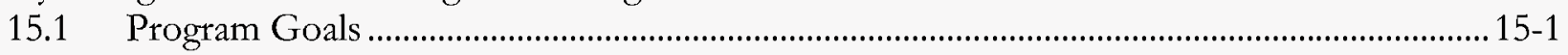

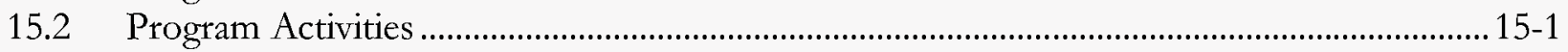

15.2.1 Hydrology and Radionuclide Investigations for Operations.................................... 15-1

15.2.2 Long-Term Groundwater Stewardship........................................................................ 15-2

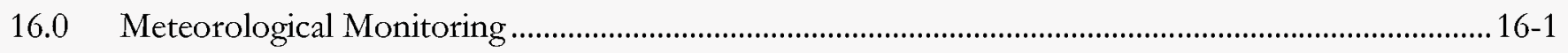

16.1 Meteorological Monitoring Goals ................................................................................... 16-1

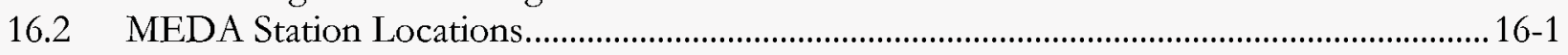

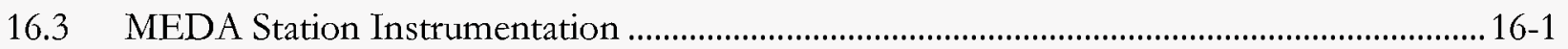

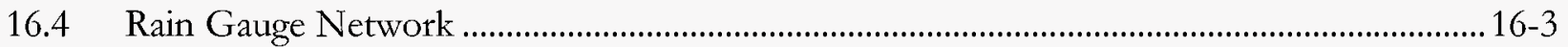

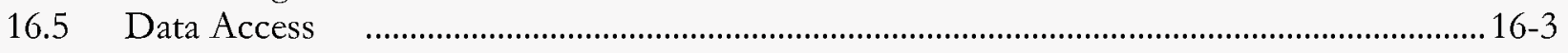

17.0 Integrated Safety Management System and Environmental Management System...........................17-1

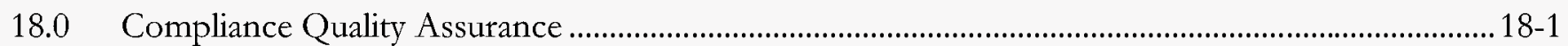

18.1 Data and Measurement Quality Objectives ............................................................................ 18-1

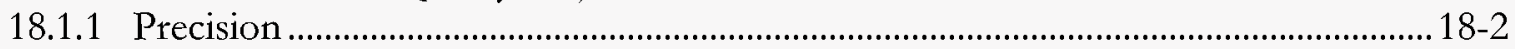

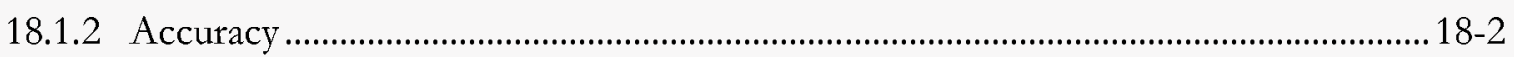

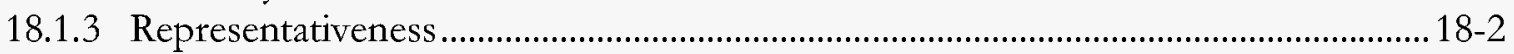

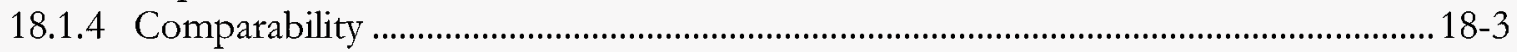

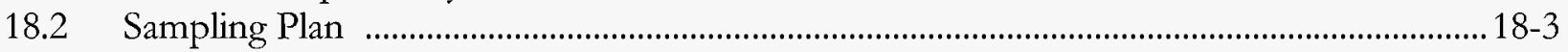

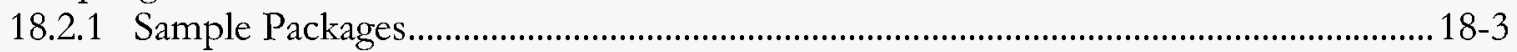

18.2.2 Database Support................................................................................................... 18-3

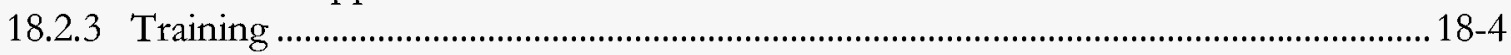


18.3 Laboratory Sample Analyses ...................................................................................... 18-4

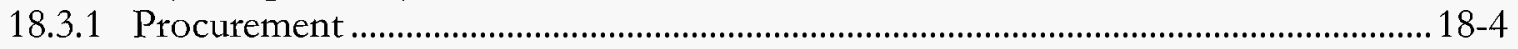

18.3.2 Initial and Continuing Assessment............................................................................. 18-5

18.3.3 Laboratory Quality Assurance Plan............................................................................18-5

18.3.3.1 LQAP Requirements .................................................................................. 18-5

18.3.3.2 LQAP Management Responsibilities ................................................................18-6

18.3.3.3 Additional Subcontract Requirements .........................................................18-6

18.4 Data Management Procedures................................................................................................... 18-8

18.5 Data Review and Systematic Assessments............................................................................ 18-8

18.5.1 Data Checks..........................................................................................................18-8

18.5.2 Data Verification.............................................................................................. 18-8

18.5.3 Data Validation......................................................................................................... 18-8

18.5.4 Data Quality Assessment .................................................................................................18-9

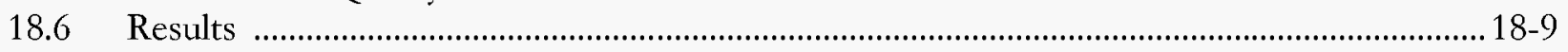

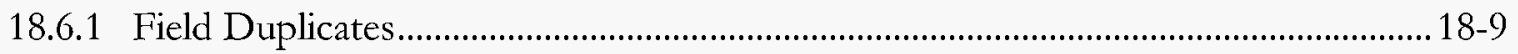

18.6.2 Laboratory Control Samples ......................................................................................18-11

18.6.3 Blank Analysis...............................................................................................18-11

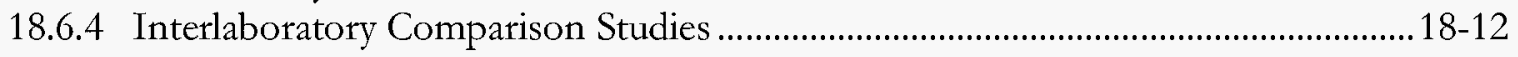

19.0 Oversight Quality Assurance Program for CEMP...............................................................................19-1

19.1 Data Quality Objectives (DQOs) .............................................................................................. 19-1

19.2 Measurement Quality Objectives (MQOs) ……………………………………………………....19-1

19.3 Sampling QA Program.............................................................................................................19-1

19.4 Laboratory QA Oversight ........................................................................................ 19-2

19.4.1 Procurement ................................................................................................................19-2

19.4.2 Initial and Continuing Assessment..............................................................................19-2

19.4.3 Laboratory QA Program................................................................................................. 19-3

19.5 Data Review................................................................................................................ 19-3

19.6 QA Program Assessments...................................................................................................... 19-4

19.72004 Sample QA Results ............................................................................................ 19-4

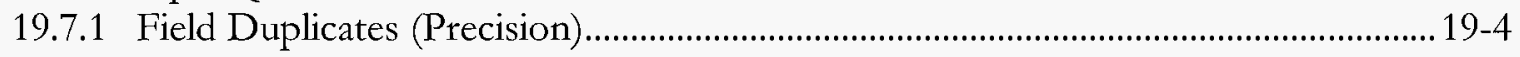

19.7.2 Laboratory Control Samples (Accuracy) …………………………………………....19-4

19.7.3 Blank Analysis....................................................................................................... 19-5

19.7.4 Interlaboratory Comparison Studies ............................................................................19-6

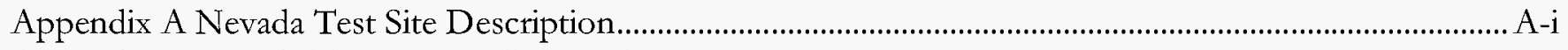

Appendix B Nevada Test Site Satellite Facilities ...……………………………………………………………… B-i

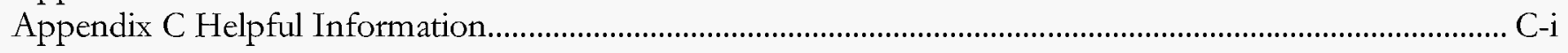

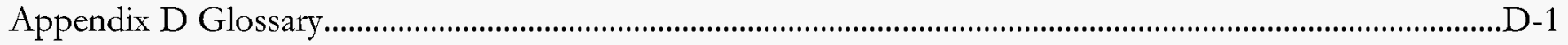

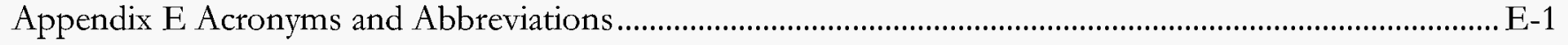

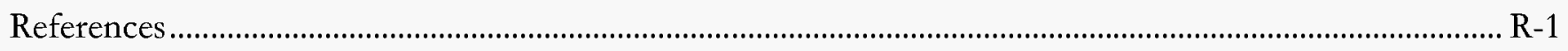

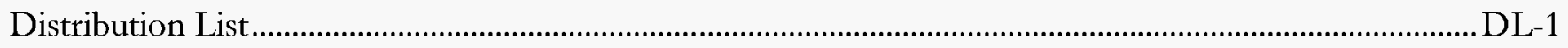




\section{List of Tables}

Table 2-1. NTS compliance status with applicable air quality regulations.................................................2-4

Table 2-2. NTS compliance status with applicable water quality and protection regulations ...................... 2-8

Table 2-3. NTS compliance status with regulations for radiation protection of the public and the environment.

Table 2-4. NTS compliance status with applicable waste management and environmental restoration regulations

Table 2-5. NTS compliance status with applicable regulations for hazardous substance control and management.

Table 2-6. NTS NEPA compliance activities conducted in 2004.

Table 2-7a. NTS compliance status with applicable pollution prevention/waste minimization regulations

Table 2-7b. NTS compliance status with the Secretary of Energy's pollution prevention and energy efficiency leadership goals.....

Table 2-8. NTS compliance status with historic preservation regulations.

Table 2-9. NTS compliance status with applicable biota and wildlife habitat regulations...........................2-30

Table 2-10. NTS compliance status with Environmental Management System regulations........................ 2-32

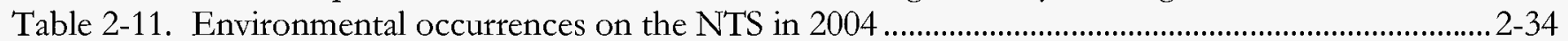

Table 2-12. Environmental permits required for NTS and NTS site facility operations...............................2-35

Table 3-1. Regulatory concentration limits for radionuclides in air............................................................ 3-4

Table 3-2. Concentrations of ${ }^{241} \mathrm{Am}$ in air samples collected in 2004 .......................................................3-6

Table 3-3. Concentrations of ${ }^{137} \mathrm{Cs}$ in air samples collected in 2004 .......................................................... 3-8

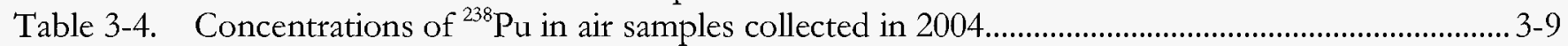

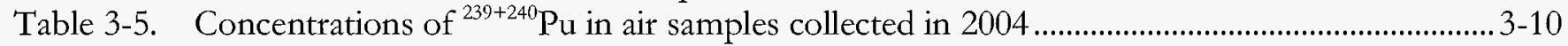

Table 3-6. Concentrations of uranium isotopes in air samples collected in 2004 ..................................... 3-12

Table 3-7. Expected ratios of uranium isotopes by type of source.......................................................... 3-13

Table 3-8. Mean uranium isotope ratios from air samples collected in 2004 ............................................ 3-14

Table 3-9. Concentrations of tritium in air samples collected in 2004 ...................................................... 3-15

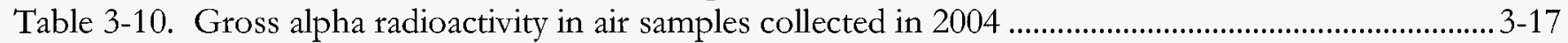

Table 3-11. Gross beta radioactivity in air samples collected in 2004 ...................................................3-18

Table 3-12. Sum of percents of compliance levels for radionuclides detected at critical receptor

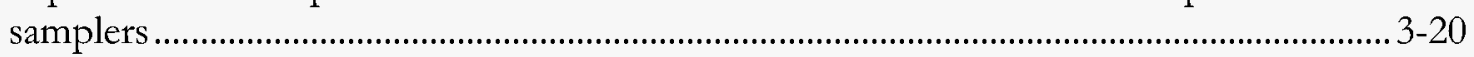

Table 3-13. NTS radiological atmospheric releases for calendar year 2004 ................................................3-21

Table 3-14. Tons of criteria air pollutant emissions released on the NTS in 2004 .....................................3-24

Table 3-15. Criteria air pollutants and HAPS released on the NTS since 1995 ........................................... 3-25

Table 3-16. Chemicals released during tests conducted at the Area 5 NPTEC in 2004 ............................ 3-27

Table 3-17. Chemicals released during tests conducted at the Area 25 Test Cell C facility in 2004 ............ 3-28

Table 4-1. Gross alpha, gross beta, tritium, and radium analysis results for offsite wells in 2004 ............... 4-8

Table 4-2. Gross alpha, gross beta, tritium, and radium analysis results for offsite springs in 2004 ........... 4-9

Table 4-3. Gross alpha, gross beta, tritium, and radium analysis results for NTS potable water supply wells

Table 4-4. Gross alpha, gross beta, and tritium analysis results for NTS monitoring wells in 2003 .......... 4-15

Table 4-5. Radiological results for E Tunnel Pond effluent pertaining to Water Pollution Control

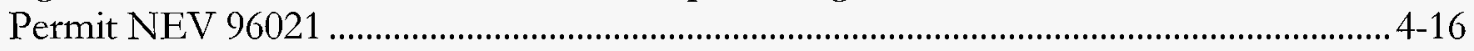

Table 4-6. Routine radiological water monitoring results for E-Tunnel Ponds in 2004 ............................ 4-17

Table 4-7. Tritium water monitoring results for NTS sewage lagoons in 2004 .......................................... 4-19 
Table 4-8. Tritium concentrations in UGTA hot wells sampled in 2004 4-19

Table 4-9. Water quality monitoring parameters and sampling design for NTS public drinking water systems

Table 4-10. Water quality analysis results for NTS public drinking water systems in 2004

Table 4-11. Water quality analysis results for NTS sewage lagoon influent waters in 2004

Table 4-12. Water toxicity analysis results for NTS sewage lagoon pond water in 2004

Table 4-13. Groundwater analysis results for NTS groundwater monitoring well SM-23-1 in 2004 ........4-31

Table 5-1. Annual direct radiation exposure rates measured at TLD locations on the NTS in 2003.......... 5-4

Table 5-2. Summary statistics for annual direct radiation exposure by TLD location type ........................... 5-7

Table 6-1. Gross alpha results for the CEMP offsite Air Surveillance Network in 2004............................. 6-4

Table 6-2. Gross beta results for the CEMP offsite Air Surveillance Network in 2004................................ 6-5

Table 6-3. TLD monitoring results for the CEMP offsite Air Surveillance Network in 2004...................... 6-7

Table 6-4. PIC monitoring results for the CEMP offsite Air Surveillance Network in 2004....................... 6-9

Table 6-5. Average natural background radiation for selected U.S. cities (excluding radon) ........................ 6-10

Table 6-6. CEMP water monitoring locations sampled in 2004................................................................6-12

Table 6-7. Tritium results for CEMP offsite springs and spring discharges in 2004................................. 6-14

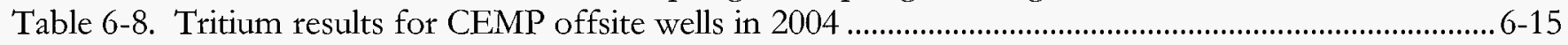

Table 7-1. Plant species sampled at Plutonium (Pu) Valley and Pu Valley Control Site in 2004................... 7-4

Table 7-2. Bats and invertebrates sampled at E Tunnel Ponds and E Tunnel Ponds

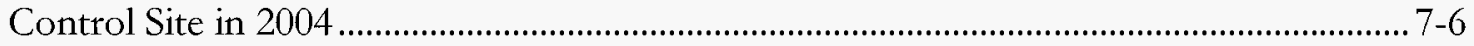

Table 7-3. Radionuclide concentrations in plants from Pu Valley and Control Site in 2004 ....................... 7-7

Table 7-4. Radionuclide concentrations in animals from Pu Valley and Control Site in 2004 ...................... 7-8

Table 7-5. Radionuclide concentrations in animals from E Tunnel Pond 5 and E Tunnel

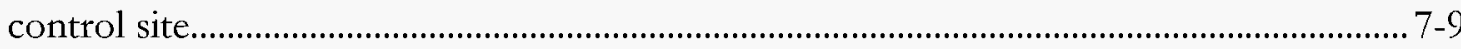

Table 8-1. Radiological atmospheric releases from NTS for 2004 used in the CAP88-PC model ................ 8-3

Table 8-2. Hypothetical annual dose to a human consuming NTS game animals sampled in 2004............. 8-6

Table 8-3. Hypothetical annual dose to a human consuming NTS game animals sampled in $2000-2003$

Table 8-4. Hypothetical worst-case dose to the MEI consuming NTS game animals based on all years of

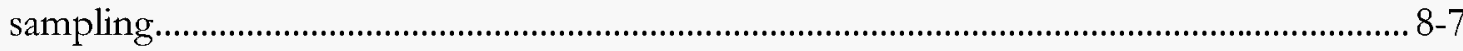

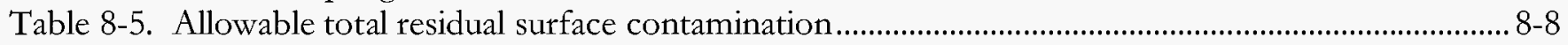

Table 8-6. Radiological dose to the general public from 2004 NTS operations .......................................... 8-9

Table 8-7. Biota dose assessment to terrestrial biota for temporary water sources created on the NTS in 2004.

Table 8-8. Site-specific dose assessment results for terrestrial plants and animals sampled on the NTS

Table 9-1. Key documents required for Area 3 RWMS and Area 5 RWMS operations.............................. 9-2

Table 9-2. Results of groundwater monitoring of UE5 PW-1, UE5 PW-2, and UE PW-3 in 2004............ 9-4

Table 9-3. Hazardous waste stored or disposed at the NTS in 2004 ........................................................... 9-7

Table 9-4. Environmental restoration activities conducted in 2004 ...........................................................9-10

Table 9-5. Quantity of solid wastes disposed in NTS landfills in CY 2004 .............................................. 9-13

Table 11-1. Volume of radioactive waste generated by year ................................................................... 11-2

Table 11-2. Mass of hazardous waste generated by year.......................................................................... 11-3 
Table 11-3. Mass of solid waste generated by year ......................................................................................11-3

Table 11-4. Volume of waste reduced through P2/WM activities by year ..................................................11-3

Table 11-5. Volume of waste reduced through P2/WM activities in 2004 ................................................11-4

Table 12-1. Summary data for cultural resources surveys, inventories, and historical evaluations

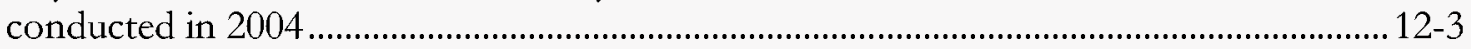

Table 12-2. Short reports, historical evaluations, technical reports, and letter reports

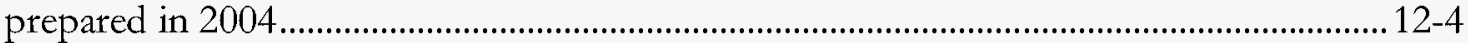

Table 12-3. Culturally affiliated tribes and organizations in the CGTO.................................................... 12-5

Table 13-1. Compliance limits and status for NTS operations in tortoise habitat...................................... 13-3

Table 13-2. Important plants which are known to occur on or adjacent to the NTS.................................. 13-4

Table 13-3. Important animals which are known to occur on or adjacent to the NTS ............................... 13-5

Table 13-4. Summary of 2004 biological survey results ................................................................................ 13-8

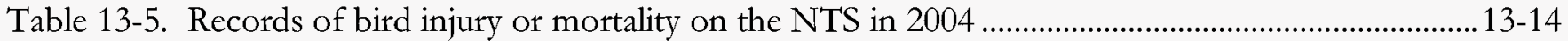

Table 18-1. Summary of field duplicate samples for compliance monitoring in 2004 ............................... 18-10

Table 18-2. Summary of laboratory control samples (LCS) for 2004 …....................................................18-11

Table 18-3. Summary of laboratory blank samples for 2004 ................................................................18-12

Table 18-4. Summary of interlaboratory comparison samples of the subcontract radiochemistry laboratories for compliance monitoring in 2004

Table 18-5. Summary of interlaboratory comparison TLD samples for the BN Radiological Health Dosimetry Group in 2004

Table 19-1. Summary of field duplicate samples for oversight monitoring in 2004.

Table 19-2. Summary of laboratory control samples (LCS) for oversight monitoring in 2004 ................... 19-5

Table 19-3. Summary of laboratory blank samples for oversight monitoring in 2004 ................................ 19-6

Table 19-4. Summary of interlaboratory comparison samples of the subcontract radiochemistry laboratory for oversight monitoring in 2004

Table 19-5. Summary of interlaboratory comparison TLD samples of the subcontract dosimetry group for compliance monitoring in 2004 
THIS PAGE INTENTIONALLY LEFT BLANK 


\section{List of Figures}

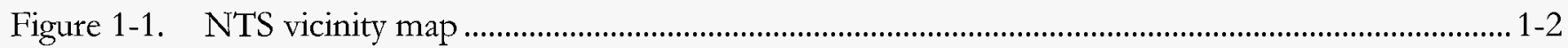

Figure 1-2. Major topographic features of the NTS ................................................................................. 1-3

Figure 1-3. NTS operational areas, principal facilities, and past nuclear testing areas................................. 1-4

Figure 3-1. Radiological air sampling network on the NTS in 2004 .........................................................3-3

Figure 3-2. Concentrations of ${ }^{241} \mathrm{Am}$ in air samples collected in 2004 ....................................................... 3-7

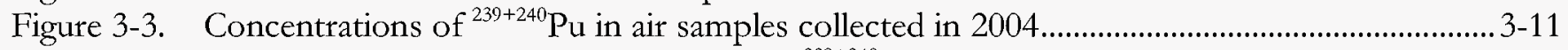

Figure 3-4. Long-term trends in highest annual mean ${ }^{239+240} \mathrm{Pu}$ for NTS area groups ............................... 3-11

Figure 3-5. Long-term trends in average annual mean ${ }^{239+240} \mathrm{Pu}$ for NTS area groups................................ 3-12

Figure 3-6. Concentrations of tritium in air samples collected in 2004 ........................................................ 3-15

Figure 3-7. Average long-term trends in tritium at locations on the NTS having at least 7 years

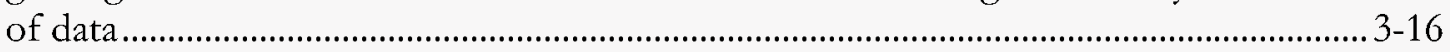

Figure 3-8. Gross alpha radioactivity in air samples collected in 2004 .................................................... 3-19

Figure 3-9. Gross beta radioactivity in air samples collected in 2004 .......................................................3-19

Figure 3-10. Criteria air pollutants released on the NTS since 1995 ....................................................... 3-25

Figure 4-1. Areas of potential groundwater contamination on the NTS .................................................... 4-2

Figure 4-2. RREMP well monitoring locations sampled on and off the NTS in 2004.............................. 4-5

Figure 4-3. RREMP spring and surface water monitoring locations sampled on and off the NTS

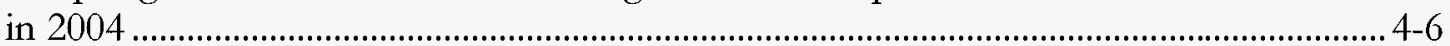

Figure 4-4. Gross alpha and gross beta levels in offsite wells from 2000 to 2004.................................. 4-8

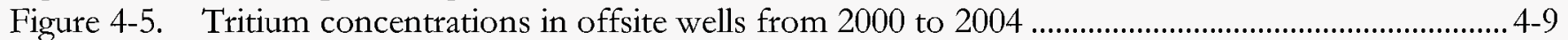

Figure 4-6. Gross alpha and gross beta levels in offsite springs from 2000 to 2004................................ 4-10

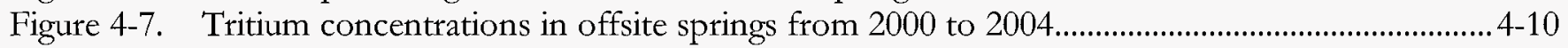

Figure 4-8. Gross alpha and gross beta levels in N'TS potable water supply wells from 2000 to

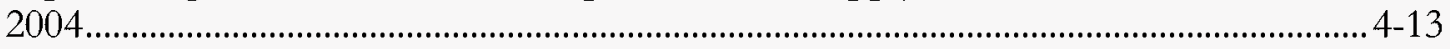

Figure 4-9. Tritium concentrations in NTS potable water supply wells from 2000 to $2004 \ldots \ldots \ldots \ldots \ldots \ldots \ldots . . . . . .4-13$

Figure 4-10. Concentrations of tritium in wells with a history of detectable levels .....................................4-16

Figure 4-11. Tritium concentration in E Tunnel Ponds from 1995 - 2004.............................................4-18

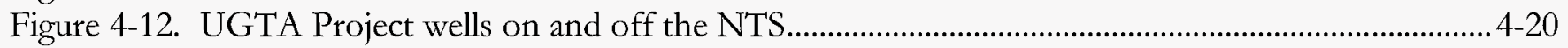

Figure 4-13. Drinking water systems on the NTS ...................................................................................4-23

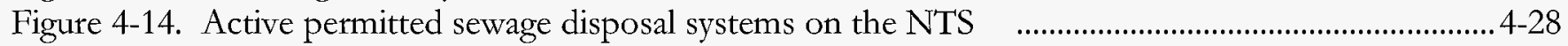

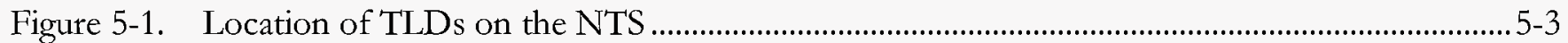

Figure 5-2. Annual exposure rates at the Area 3 RWMS during 2004........................................................5-8

Figure 5-3. Annual exposure rates at the Area 5 RWMS during 2004 ..................................................... 5-9

Figure 5-4. Annual exposure rates at B and E1 locations on the NTS in 2004 ....................................... 5-10

Figure 5-5. Trend in direct radiation exposure measured at TLD locations with at least eleven-year data histories .................................................................................... 5-11

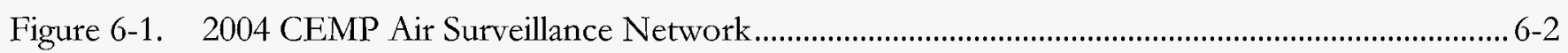

Figure 6-2. CEMP station at Beatty, Nevada ........................................................................................... 6-3

Figure 6-3. Historical trend for gross alpha analysis for all CEMP stations .............................................. 6-5

Figure 6-4. Historical trend for gross beta analysis for all CEMP stations................................................. 6-6

Figure 6-5. Historical trend for TLD analysis for all CEMP stations..................................................6-8

Figure 6-6. The effect of meteorological phenomena on background gamma readings ............................ 6-10 
Figure 6-7. 2004 CEMP water monitoring locations

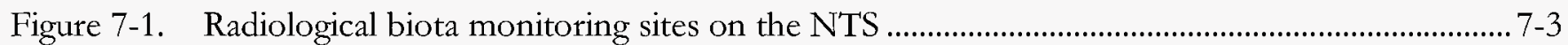

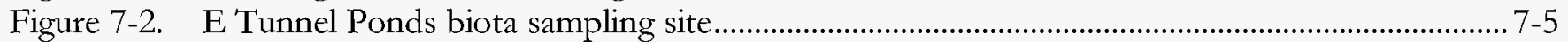

Figure 8-1. Map of the NTS showing CY 2004 CEDEs within $80 \mathrm{~km}$ of emission sources..................... 8-4

Figure 8-2. Radiation dose to MEI offsite who is not consuming game animals from the NTS................ 8-5

Figure 8-3. Comparison of radiation dose to the MEI from NTS operations and from natural

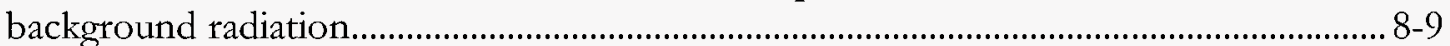

Figure 8-4. Terrestrial and aquatic dose evaluation areas for assessing potential dose to biota ................. 8-11

Figure 8-5. Results of Level 1 and Level 2 Screens for dose evaluation areas on the NTS ....................... 8-12

Figure 13-1. Desert tortoise distribution and abundance on the NTS and locations of

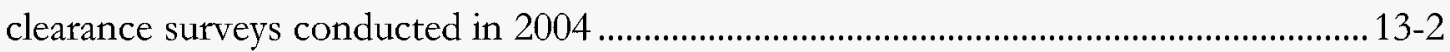

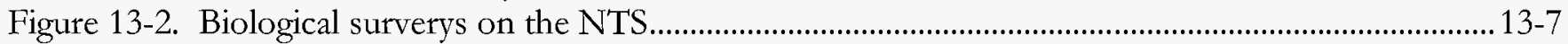

Figure 13-3. Sensitive plant populations monitored on the NTS in 2004 ...............................................13-10

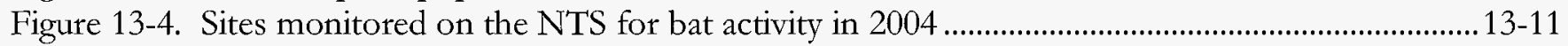

Figure 13-5. Feral horse range on the NTS and sighting locations of horses and horse sign in 2004 .

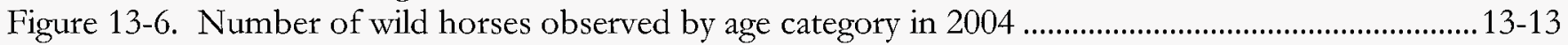

Figure 13-7. Number of bird deaths recorded on the NTS by year and by cause ...................................... 13-14

Figure 16-1. Example of a typical MEDA station with a 10 meter tower ...................................................16-1

Figure 16-2. MEDA station locations on and near the NTS ................................................................16-2

Figure 16-3. Climatological rain gauge network on the NTS ......................................................................... 16-4 


\section{Executive Summary}

The Nevada Test Site Environmental Report 2004 was prepared by Bechtel Nevada (BN) to meet the information needs of the public and the requirements and guidelines of the U.S. Department of Energy (DOE) for annual site environmental reports. This Executive Summary presents the purpose of the document, the major programs conducted at the Nevada Test Site (NTS), NTS key environmental initiatives, radiological releases and potential doses to the public resulting from site operations, a summary of non-radiological releases, implementation status of the NTS Environmental Management System, and significant environmental accomplishments. Much of the content of this Executive Summary is also presented in a separate stand-alone pamphlet titled Nevada Test Site Environmental Report Summary 2004. It was produced this year to provide a more cost-effective and wider distribution of a hardcopy summary of the Nevada Test Site Environmental Report 2004 to interested DOE stakeholders.

\section{Purpose of the NTS Environmental Report}

BN prepares this document to satisfy DOE Order 231.1A Environment, Safety and Health Reporting. It is prepared in order to (1) report compliance status with environmental standards and requirements, (2) present results of environmental monitoring of radiological and nonradiological effluents, (3) report estimated radiological doses to the public from releases of radioactive material, (4) summarize environmental incidents of noncompliance and actions taken in response to them, (5) describe the NTS Environmental Management System and characterize its performance, and (6) highlight significant environmental programs and efforts. This report meets these objectives for the NTS and its three Nevada satellite sites mentioned below.

\section{Major Site Programs}

The U.S. Department of Energy, National Nuclear Security Administration Nevada Site Office (NNSA/NSO) directs the management and operation of the NTS and seven satellite sites across the nation. The NTS is located about 105 kilometers $(\mathrm{km})$ (65 miles [mi]) northwest of Las Vegas. The seven satellite sites include three sites in Nevada (North Las Vegas Facility, Cheyenne Las Vegas Facility, and the Remote Sensing Laboratory - Nellis) and four sites in other states (Remote Sensing Laboratory - Andrews in Maryland, Livermore Operations in California, Los Alamos Operations in New Mexico, and Special Technologies Laboratory in California). Los Alamos, Lawrence Livermore, and Sandia National Laboratories are the principal organizations that sponsor and implement the nuclear weapons programs at the NTS. BN is the Management and Operations (M\&O) contractor accountable for the successful execution of work and ensuring that work is performed in compliance with environmental regulations. The NTS and its seven satellite sites all provide support to enhance the NTS as a site for weapons experimentation and nuclear test readiness. The three major NTS programs are Stockpile Stewardship, National Security Response Program and Operations, and Environmental Management.

\section{Other Key Initiatives}

Apart from the major site programs, other NTS activities include demilitarization activities, controlled spills of hazardous material at the Non-Proliferation Test and Evaluation Complex (NPTEC) (formerly known as the Hazardous Materials Spill Center) for research purposes, remediation of industrial sites, processing of waste destined for the Waste Isolation Pilot Plant in Carlsbad, New Mexico, disposal of radioactive and mixed waste, and environmental research. In addition, there are continued efforts to bring other business to the NTS, such as aerospace and alternative energy technology development and support of U.S. Department of Homeland Security National Center for Combating Terrorism. 


\section{Environmental Performance Measure Programs}

During the conduct of the major programs and other key initiatives mentioned above, NNSA/NSO complies with applicable environmental and public health protection regulations and strives to manage the land and facilities at the NTS as a unique and valuable national resource. For the identification of NTS environmental initiatives, BN relies upon BN's Integrated Safety Management System (ISMS), contractual Work Smart Standards (WSS), and the Environmental Management System (EMS). The ISMS is designed to ensure the systematic integration of environment, safety, and health concerns into management and work practices so that NTS missions are accomplished safely and in a manner which protects the environment. Implementation of an ISMS at the NTS was verified by NNSA/NSO in July 2001. NNSA/NSO oversees ISMS implementation through the Integrated Safety Management Council. Each Council member performed a self-assessment in September 2004 and verified that the ISMS continues to be effectively implemented at the NTS.

WSS are an integral part of the ISMS whereby hazards and environmental aspects of work are identified and standards of operation are established that are specific to the work environment, its associated hazards, and its threats to the environment. WSS are developed at the management level with the most expertise in the work. NNSA/NSO approved the initial complete set of BN WSS in September 1996. The approved WSS identify within each BN program the contractual commitment to meet applicable laws, regulations, and policies which protect the public and the environment. Compliance with WSS is tracked through management assessments.

In 2000, Executive Order (EO) 13148 Greening of the Govermment Through Leadership in Environmental Management was issued. This EO requires all federal agencies to adopt an environmental management system (EMS). EMSs are designed to incorporate concern for environmental performance throughout an organization, with the ultimate goal being continual reduction of the organization's impact on the environment. DOE requires contractors who operate DOE sites to develop an EMS and expects full integration of their EMS into their ISMS by December 2005. In 2004, the ISMS Program Plan was updated to specify that the EMS and DOE Order 450.1 are the method by which the environmental part of ISMS is implemented (see Section 17.0). A committee during 2004 was formed that identified priority areas of improvement (Objectives) and is starting to identify organization specific goals (Targets) within these priority areas. These Targets and Objectives will be approved by the Executive Safety Committee, and the progress will be tracked and reported. During 2004, the employee environmental awareness program was expanded. Copies of the revised Environmental Policy were mailed to BN organizations, posted on bulletin boards and the BN intranet home page. Articles about the new Policy and the EMS were put in BN employee publications, and a section on environmental issues was added to the BN project manager training course. Full integration of EMS into ISMS should be complete by the deadline of December 31, 2005.

\section{Performance Measures}

Performance measures are used to evaluate the achievement of organization or process goals and to identify the need to institute changes in an organization or process. The NTS performance measures, defined from the WSS, relate to protection of the environment and the public from effects of NTS operations. These performance measures apply to several programs and processes. They include (1) the potential radiological dose received by the maximally exposed offsite individual, (2) the identification, notification, and mitigation of spills and releases to the environment, (3) the reduction in the generation of wastes, and (4) compliance with applicable environmental protection regulations. The performance measures tracked by each process or program (e.g., air quality protection) are consolidated and presented in this report in Section 2.0, Compliance Summary.

\section{Offsite Radiological Releases into Air}

An oversight radiological air monitoring program is run by the Community Environmental Monitoring Program (CEMP) and is coordinated by the Desert Research Institute (DRI) of the University and Community College System of Nevada under contract with NNSA/NSO (see Section 6.0). Its purpose is to provide monitoring for radionuclides that might be released from the NTS. A network of 26 CEMP stations, located in selected towns and communities within $386 \mathrm{~km}$ (240 mi) from the NTS, was operated continuously during 2004. The CEMP stations monitored gross 
alpha and beta radioactivity in airbome particulates using low-volume particulate air samplers, penetrating gamma radiation using thermoluminescent dosimeters (TLDs), gamma radiation exposure rates using pressurized ion chamber (PIC) detectors, and meteorological parameters using automated weather instrumentation.

No airborne radioactivity related to historic or current NTS operations was detected in any of the samples from the CEMP particulate air samplers during 2004. Gross alpha and gross beta radioactivity was detected at all CEMP stations at levels which were consistent with previous years and which reflect radioactivity from naturally-occurring radioactive materials (see Section 6.1.1.1). No man-made gamma-emitting radionuclides were detected.

TLD and PIC detectors measure gamma radiation from all sources: natural background radiation from cosmic and terrestrial sources and man-made sources. The offsite TLD and PIC results remained consistent with previous years' background levels and are well within background levels observed in other parts of the United States.

The highest total annual gamma exposure measured offsite, based on PIC data, was 178 milliroentgen $(\mathrm{mR})$ at Milford, Utah. The lowest offsite gamma exposure rate measured was $67 \mathrm{mR}$ per year (yr) at Pahrump, Nevada (see Section 6.1.3).

\section{Onsite Radiological Releases into Air}

A network of 19 air sampling stations (three having low-volume particulate air samplers, one having a tritium water vapor sampler, and 15 having both) and a network of 107 TLDs were used to monitor onsite NTS radioactive emissions in 2004 (see Section 3.1). The 2004 monitoring results were also used, in conjunction with U.S. Environmental Protection Agency (EPA)-approved mathematical models, to calculate the radiological dose to the public residing within $80 \mathrm{~km}$ (50 mi) of the NTS. There were minimal radioactive air emissions from current NTS projects in 2004 which came from only one NTS facility: Building 650 in Mercury, Area 23. A total of 0.000042 Curies (Ci) of tritium gas was released at Building 650 during the calibration of laboratory equipment (see Section 8.1,2).

Gross alpha and gross beta radioactivity was detected at all stations on the NTS, but no increasing trend in levels of radioactivity was observed at any station (see Section 3.1.4.6). The highest average gross alpha and gross beta activities were seen at Sugar Bunker, an unoccupied structure used during past nuclear testing, located about $1 \mathrm{~km}$ (0.6 mi) south-southwest of the Area 5 Radioactive Waste Management Complex (RWMC). The lowest average gross alpha and beta activities were measured at the Little Feller 2 air sampler in Area 18 and the 3545 Substation air sampler in Area 16, respectively. The mean gross alpha concentrations were slightly higher at the locations near sites with known deposits of radioactivity from past nuclear tests in Areas 1, 3, 5, 6, 9, 10, and 20. The mean gross beta concentrations varied less by location throughout all NTS areas. Both the weekly gross alpha and gross beta concentrations continued to show a general temporal variation that was common for all locations.

Background gamma radiation exposure rates on the NTS, measured at TLD stations located away from radiologically contaminated sites, ranged from 60 to $156 \mathrm{mR} / \mathrm{yr}$ during 2004 (see Section 5.3). Direct gamma radiation exposure to the public from NTS operations was negligible (see Section 5.3.1). Areas accessible to the public (e.g., the NTS entrance gate) had exposure rates comparable to natural background rates, with one exception. During the fourth quarter of 2004, the daily average gamma radiation exposure rate measured on the west side of the parking area outside the NTS entrance gate rose to $358 \mathrm{mR} / \mathrm{yr}$. It is likely that waste shipments entering the NTS were responsible for this increase in the fourth quarter. Radionuclide contamination at legacy sites has resulted in localized elevated gamma exposure rates, but the public has no access to these sites nor are there NTS personnel working in these areas. The highest exposure rate at monitored locations was $888 \mathrm{mR} / \mathrm{yr}$ at Schooner, one of the legacy Plowshare sites on Pahute Mesa (see Table 5-1). Sixteen TLD stations monitor the RWMC in Areas 3 and 5 (see Section 5.3.2). The mean gamma exposure rate at these stations was $148 \mathrm{mR} / \mathrm{yr}$; exposure rates ranged from 106 to $401 \mathrm{mR} / \mathrm{yr}$. The public is not allowed unsupervised access to these sites.

Several man-made radionuclides from legacy contamination were measured in air samples at levels above their minimum detectable concentrations (MDCs) in 2004: americium-241 $\left({ }^{241} \mathrm{Am}\right)$; tritium $\left({ }^{3} \mathrm{H}\right)$; and plutonium isotopes ${ }^{238} \mathrm{Pu}$ and ${ }^{239+240} \mathrm{Pu}$ ) (see Section 3.1.4). These were attributed to the resuspension of contamination in surface soils from legacy sites and to the evaporation and transpiration of tritium from the soil, plants, and containment ponds at legacy sites. The highest mean level of ${ }^{241} \mathrm{Am}\left(48.07 \times 10^{-18}\right.$ micro-curies per milliliter $\left.[\mu \mathrm{Ci} / \mathrm{mL}]\right)$ was detected at Bunker 9-300 in Area 9, a vacant building located within an area of known soil contamination from past nuclear tests. 
The highest mean level of tritium $\left(364.69 \times 10^{-6}\right.$ picocuries $\left.[\mathrm{pCi}] / \mathrm{mL}\right)$ was detected at Schooner, site of the secondhighest yield Plowshare cratering experiment on the NTS where tritium-infused ejecta surrounds the crater. The highest mean levels of plutonium isotopes in air were at Bunker $9-300\left(5.61 \times 10^{-18} \mu \mathrm{Ci} / \mathrm{mL}\right.$ for ${ }^{238} \mathrm{Pu}$ and $294.12 \times 10^{-18} \mu \mathrm{Ci} / \mathrm{mL}$ for $\left.{ }^{239+240} \mathrm{Pu}\right)$. The relatively high plutonium values occur most often at the Bunker 9-300 air sampling station, due to historical nuclear testing in Area 9 and surrounding Areas 3, 4, and 7. Uranium isotopes are also detected in air samples collected in areas where depleted uranium ordnance have been used or tested. However, the samples' isotopic ratios were what one would expect from naturally-occurring uranium in soil and not from either man-made depleted or enriched uranium.

Both ${ }^{239+240} \mathrm{Pu}$ and tritium continued to show overall decreasing trends in concentrations at air sampler sites (see Section 3.1.4.3 and Section 3.1.4.5). The decrease in tritium air concentrations is a result of the cessation of testing in 1992 (no additional releases), of radioactive decay (half-life of tritium is 12 years), and of its depletion from the soil over the years due to evaporation and transpiration (uptake and release of water through plants). Annual mean tritium concentrations, grouped by NTS administrative areas, have dropped a factor of one thousand for all areas except Area 20 (where Schooner is located). The gradual decrease in plutonium concentrations in air over time is attributed to its dispersal by wind and its weathering in the ground where it is bound to less mobile particles.

\section{Offsite Radiological Monitoring of Water}

Offsite water monitoring conducted annually by BN (see Section 4.1), as well as by DRI through the CEMP (see Section 6.2), verifies that there has been no offsite migration of man-made radionuclides from NTS underground contamination areas.

In 2004, BN conducted radiological monitoring of 14 offsite wells and 2 offsite springs. The 14 wells include 6 private domestic and local community wells and 8 NNSA/NSO wells drilled for hydrogeologic investigations including groundwater flow modeling. All of the BN-sampled wells and springs are in Nevada within $18.6 \mathrm{mi}(30 \mathrm{~km})$ from the western and southern borders of the NTS. The DRI, through the CEMP, sampled 24 offsite sampling locations in 2004. They include 17 wells, 3 water supply systems, and 4 springs located in selected towns and communities within $240 \mathrm{mi}$ from the NTS. One site, the Beatty Water and Sewer well, is sampled by both BN and CEMP.

CEMP and BN water samples are both analyzed for tritium. To be able to detect the smallest possible amounts of tritium in offsite water supplies, enriched tritium analyses were run on all samples. The MDC for tritium using this enrichment process was approximately $25 \mathrm{pCi} / \mathrm{L}$ and $26 \mathrm{pCi} / \mathrm{L}$ for the $\mathrm{BN}$ and the CEMP samples, respectively. Without enrichment, the MDC for tritium typically ranges from $200-400 \mathrm{pCi} / \mathrm{L}$. To put these values in perspective, the drinking water Maximum Contaminant Level (MCL) for tritium is $20,000 \mathrm{pCi} / \mathrm{L}$.

BN offsite water samples are also analyzed for man-made gamma-emitting radionuclides that would signify contamination from nuclear testing and for gross alpha and gross beta activity to determine if alpha or beta activity at any well or spring are increasing over time.

CEMP results this year, as in past years, continue to verify that no contaminated groundwater has migrated beyond the NTS boundaries into surrounding water supplies used by the public. Samples from only two locations, Boulder City and Henderson municipal water supplies, contained tritium at levels barely above detection (see Section 6.2.4). All other wells had non-detectable levels of tritium. These two municipal water systems obtain water from Lake Mead, which has documented levels of residual tritium persisting in the environment that originated from global atmospheric nuclear testing.

Similarly, the results of BN offsite water monitoring verified that there has been no offsite migration of man-made radionuclides from NTS underground contamination areas. BN detected tritium in only one offsite well, PM-3 at $20 \mathrm{pCi} / \mathrm{L}$, slightly above the sample-specific MDC (see Section 4.1.4). A duplicate sample from PM-3 collected on the same date was below the sample-specific MDC. All offsite well and spring samples contained detectable gross alpha and gross beta activity which are believed to come from natural sources. Gross alpha was found at levels which exceeded drinking water standards at only one offsite monitoring well, ER-OV-02. This well is an NNSA/NSO well that is not used for drinking water and is closed to the public. This well produces water from a volcanic aquifer that 
may have relatively high quantities of natural alpha-yielding elements in the host rock. All gross beta concentrations in samples from offsite wells sampled by BN were less than the EPA Level of Concern for drinking water. No offsite wells contained any man-made gamma-emitting radionuclides.

\section{Onsite Radiological Releases into Water}

Radioactivity in onsite groundwater and surface waters on the NTS is monitored to (1) ensure that NTS drinking water is safe, (2) determine if permitted facilities on the NTS are in compliance with permit discharge limits for radionuclides, (3) estimate radiological dose to onsite wildlife using natural and man-made water sources, and (4) verify that groundwater is being protected from disposed radioactive wastes at the Area 3 and Area 5 Radioactive Waste Management Sites (RWMSs). In 2004, the onsite monitoring network was comprised of 10 potable water supply wells, 12 monitoring wells (which include 3 compliance wells for the Area 5 RWMS and 1 compliance well for the Area 23 sewage lagoon), 1 tritiated water containment pond system, and 2 sewage lagoons.

The 2004 data continue to indicate that underground nuclear testing has not impacted the NTS potable water supply network (see Section 4.1.6). All of the water samples from the ten supply wells had non-detectable concentrations of tritium and man-made gamma-emitting radionuclides. The gross alpha and gross beta radioactivity detected in potable water supply wells represent the presence of naturally-occurring radionuclides.

Four onsite monitoring wells (PM-1, U-19BH, UE-7NS, and WW A) had detectable concentrations of tritium in 2004 ranging from $23 \pm 14$ to $475 \pm 27 \mathrm{pCi} / \mathrm{L}$, all well below the MCL of $20,000 \mathrm{pCi} / \mathrm{L}$ (see Section 4.1 .7 ). Each of the four monitoring wells is located within $1 \mathrm{~km}(0.6 \mathrm{mi})$ of an historical underground nuclear test; all have consistently had detectable levels of tritium in past years. There were also tritium levels above the MDC from three wells sampled to validate performance of a radioactive waste disposal pit at the Area 5 RWMS (UE5PW-1, UE5PW-2, and UE5PW-3). Tritium concentrations ranged from $30 \pm 12$ to $37 \pm 13 \mathrm{pCi} / \mathrm{L}$ in these wells; in each case duplicate samples collected at the same times from each well had measured tritium levels below their MDCs.

Five constructed basins collect and hold water discharged from $\mathrm{E}$ Tunnel in Area 12, where nuclear testing was conducted in the past. Tunnel effluent water and sediment samples are analyzed for tritium, gross alpha, gross beta, and other radionuclides. Most samples had detectable radionuclide concentrations in 2004 (see Section 4.1.8). The average tritium concentration in tunnel effluent water was $710,000 \mathrm{pCi} / \mathrm{L}$, lower than the limit allowed under a discharge permit $(1,000,000 \mathrm{pCi} / \mathrm{L})$. Gross alpha and gross beta values in 2004 were also less than their permitted limits: $13.4 \mathrm{pCi} / \mathrm{L}$ with a permissible limit of $35.1 \mathrm{pCi} / \mathrm{L}$ for gross alpha and $72 \mathrm{pCi} / \mathrm{L}$ with a permissible limit of $101 \mathrm{pCi} / \mathrm{L}$ for gross beta.

Tritiated water is also pumped into lined sumps during studies conducted by the Underground Test Area (UGTA) Project. To characterize the groundwater regime under the NTS, suitable wells are being drilled and existing wells recompleted in the vicinity of certain underground tests and at other locations on the NTS designated by hydrologists. During these drilling operations, if the tritium level exceeds $200,000 \mathrm{pCi} / \mathrm{L}$, contaminated water is pumped from the wells and diverted to lined containment ponds, as required by the state. During 2004, water containing tritium was pumped from Wells U-3cn PS\#2, U-19ad PS\#1, ER-20-5 \#1, and ER-20-5 \#3 into lined containment ponds. Levels of tritium in these ponds ranged from $113,000 \mathrm{pCi} / \mathrm{L}$ at ER-20-5 \#3 to 38,000,000 $\mathrm{pCi} / \mathrm{L}$ at ER-20-5 \#1 (see Section 4.1.10).

\section{Estimated Radiation Dose to the Public}

Man-made radionuclides from past nuclear testing have not been detected in offsite groundwater in the past or during 2004. The only pathways, therefore, by which the offsite public could receive a radiation dose from NTS operations are from inhalation and ingestion. 
Measured Background NTS Radiation Dose - Background gamma radiation exposure rates on the NTS were measured at eight TLD stations located away from radiologically contaminated sites; these ranged from 0.16 to $10.43 \mathrm{mR} /$ day during 2004 (see Section 5.3). This equates to an annual estimated background external dose of 60 to 156 millirem per year $(\mathrm{mrem} / \mathrm{yr})^{1}$ to a hypothetical person residing at those locations all year.

Inhalation Pathway - The radiation dose to the general public via just the air transport pathway was estimated using the air sampling results from six onsite EPA-approved "critical receptor" sampling stations. Among these six stations, the Schooner air station in the far northwest corner of the NTS experienced the highest concentrations of radioactive air emissions (see Section 31.5). An individual residing at this station would experience a dose from air emissions of $2.5 \mathrm{mrem} / \mathrm{yr}$. This dose is less than the limit of $10 \mathrm{mrem} / \mathrm{yr}$. No one resides at this location, of course; the dose at offsite populated locations $20-80 \mathrm{~km}$ from the Schooner station would be much lower due to wind dispersion.

Inhalation and Ingestion Pathway - The radiation dose to the general public from inhalation and ingestion of airborne radioactive contaminants was estimated using the air sampling results and air transport models. Estimates of radionuclide emissions from the following sources were used to compute total air emissions from source locations on the NTS: (1) NTS facilities; (2) the resuspension of legacy deposits of radionuclides in NTS soil; (3) the transpiration and evaporation of tritium at sites of past nuclear tests; and (4) the evaporation of tritium from ponds used to contain tritium-contaminated groundwater. The radiation dose to the general public is expressed as the effective dose equivalent [EDE]) to the maximally-exposed individual (MEI); this was computed to be $0.12 \mathrm{mrem} / \mathrm{yr}$ $(0.0012 \mathrm{mSv} / \mathrm{yr}$ ) for a resident of Cactus Springs, Nevada (see Section 8.1.3). This is well below the dose limit of $10 \mathrm{mrem} / \mathrm{yr}$ specified by the National Emission Standards for Hazardous Air Pollutants under the Clean Air Act. This dose is consistent with those calculated for past years.

Ingestion Pathway for Radionuclides in Game Animals - Game animals from different contaminated NTS sites are trapped each year and analyzed for their radionuclide content. These results are used to construct worst-case scenarios for the dose to hunters who might consume these animals if the animals moved off the NTS. NTS game animals include pronghorn antelope, mule deer, chukar, Gambel's quail, mourning doves, cottontail rabbits, and jackrabbits. The MEI who is a hunter is assumed to eat 20 doves, 20 quail, 20 chukar, 20 jackrabbits, and 1 pronghorn antelope in a year. It is also assumed that the dose from each animal consumed is the average calculated dose for that species which was sampled from the NTS location where the highest levels of radionuclides in that species' muscle tissues were found. The resultant potential dose from consuming NTS game animals representative of those sampled is $0.39 \mathrm{mrem} / \mathrm{yr}$ (see Section 8.1.4). To put this dose into perspective, it is less than the dose from cosmic radiation received by an individual while on a one-hour plane ride at 39,000 ft.

All Possible Pathways - The hypothetical MEI was also assumed to be a hunter who harvested NTS game animals and received the additional radiation dose of $0.39 \mathrm{mrem} / \mathrm{yr}$. The resultant total radiation dose to the MEI attributable to NTS operations from all possible pathways combined was $0.51 \mathrm{mrem} / \mathrm{yr}(0.0051 \mathrm{mSv} / \mathrm{yr}$ (see Section 8.1.6) This dose is a very small fraction ( 0.15 percent) of the total radiation dose from naturally-occurring sources. This total dose is also well below the dose limit of $100 \mathrm{mrem} / \mathrm{yr}$ established by DOE Order 5400.5 Radiation Protection of the Public and the Environment.

The collective population dose within $80 \mathrm{~km}(50 \mathrm{mi})$ of the emission sources was estimated to be 0.47 person-rem/yr $(0.0047$ person-Sv/yr) (see Section 8.1.7).

\footnotetext{
${ }^{1}$ Direct radiation exposure is usually measured in the unit milli-roentgen $(\mathrm{mR})$, which is a measure of exposure in terms of a specified number of ionizations in air. Generally, the dose resulting from an exposure from the most common external radionuclides can be approximated by equating a $1 \mathrm{mR}$ exposure with a $1 \mathrm{mrem}(0.01 \mathrm{mSv})$ dose.
} 


\section{Estimated Radiation Dose to the General Public from the NTS in 2004}

\begin{tabular}{|c|c|c|c|c|c|}
\hline Pathway & $\begin{array}{l}\text { Dose to I } \\
\text { Exposed I } \\
\text { (mrem/yr) }\end{array}$ & $\begin{array}{l}\text { Iaximally } \\
\text { ndividual } \\
\text { (mSv/yr) }\end{array}$ & $\begin{array}{l}\text { Percent of DOE } \\
\text { 100-mrem/yr } \\
\text { Limit }\end{array}$ & $\begin{array}{l}\text { Estimated } \\
\text { Populatic } \\
\text { (person-rem/yr) }\end{array}$ & $\begin{array}{l}\text { Collective } \\
\text { on Dose }{ }^{(a)} \\
\text { (person-Sv/yr) }\end{array}$ \\
\hline Air & 0.12 & 0.0012 & 0.12 & $0.47^{(a)}$ & 0.0047 \\
\hline Water & 0 & 0 & 0 & 0 & 0 \\
\hline Wildlife & 0.39 & 0.0039 & 0.39 & $U^{(b)}$ & U \\
\hline All Pathways & 0.51 & 0.0051 & 0.51 & $0.47^{(c)}$ & 0.0047 \\
\hline
\end{tabular}

(a) Sum of radiation doses from all emission sources at each populated location within $80 \mathrm{~km}$ of emission sources multiplied by the population at each location, and then summed over all locations.

(b) Unable to make this estimate due to a lack of data on number of game animals harvested near the NTS by hunters in 2004 .

(c) The dose contribution from wild life is not included. It is likely to be negligible when averaged over the entire population within an $80-\mathrm{km}$ radius.

\section{Comparison of Radiation Dose to the MEI and the Natural Radiation Background (Percent of Total)}

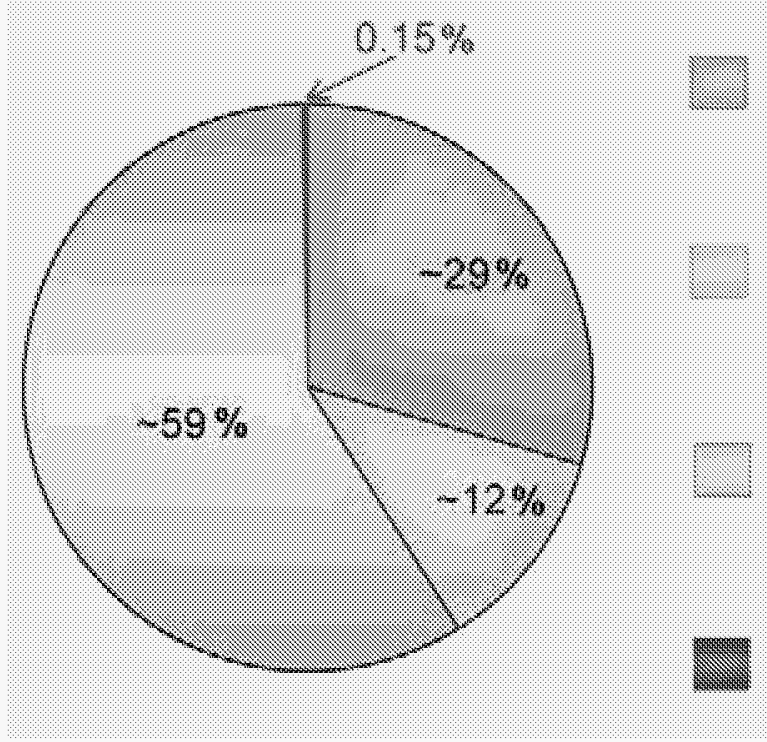

Doste tronn cosmitic and: tarrestrial radiation roteasurend by PIC at Indians Springs. NW, 100 meremily

Doser trom natural radianuclides in the body. 48 mirentyt

Doste tron intalation of

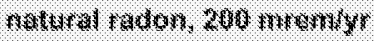

Dosas trom calculated NTS arrissions and

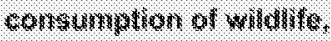
0.51 mrentiyr

\section{Onsite Non-Radiological Releases into Air}

There were no discharges of non-radiological hazardous materials to offsite areas in 2004. Therefore, only onsite non-radiological environmental monitoring of NTS operations was conducted. Air quality was monitored on the NTS throughout the year as required by state of Nevada permits for those operations that release criteria air pollutants, hazardous air pollutants (HAPs), or toxic and hazardous chemicals. The NTS has been issued a Class II air permit from the state of Nevada. Class II permits are issued to facilities which emit small quantities of air pollutants within a year (less than 100 tons of each criteria air pollutant, or 10 tons of any one HAP, or 25 tons of any combination of HAPs). 
An estimated 6.91 tons of criteria air pollutants were released on the NTS in 2004. They included: particulate matter equal to or less than 10 microns in diameter (PM10), carbon monoxide (CO), nitrous oxides $(\mathrm{NO})$, sulfur dioxide $\left(\mathrm{SO}_{2}\right)$, and volatile organic compounds (VOCs) (see Section 3.2.1). The majority of these emissions (4.60 tons) were VOCs. The NPTEC facility, where controlled spills of hazardous materials are conducted, produced more VOCs than all other permitted NTS facilities. Total air emissions of lead, also a criteria pollutant, in 2004 was 10.4 pounds. The quantity of HAPS released in 2004 was 0.41 tons. No emission limits for any criteria air pollutants of HAPS were exceeded. On May 12, 2004, the state of Nevada conducted an inspection of the following facilities regulated by the NTS air quality permit: the Area 1 Aggregate Plant, Area 1 Batch Plant, and the NPTEC. There were no findings or exceedances of permit limits.

Asbestos is the only non-radiological HAP of regulatory concern on the NTS. In 2004, all materials containing regulated asbestos that were removed from NTS facilities were disposed of in the Mercury landfill. The quantities removed did not exceed EPA's notification threshold (see Section 3.2.7). The Mercury landfill documented receipt of 15 tons of such material in 2004.

A combined total of 5 tests consisting of 25 releases of hazardous chemicals were conducted at the Area 5 and the Area 25 Test Cell C NPTEC facilities in 2004 (see Section 3.2.4). An annual report of the types and amounts of chemicals released and the test plans and final analysis reports for each chemical release were submitted to the state. No ecological monitoring was performed since each test posed a very low level of risk to the environment and biota.

\section{Onsite Non-Radiological Releases into Water}

There are no liquid discharges to navigable waters, offsite surface water drainage systems, or publicly owned treatment works resulting from operations on the NTS. Therefore, no Clean Water Act National Pollution Discharge Elimination System (NPDES) permits are required for NTS operations.

In 2004, industrial discharges on the NTS were limited to two operating sewage lagoon systems, the Area 6 Yucca Lake and Area 23 Mercury systems. Under the conditions of state of Nevada operating permits, liquid discharges to these sewage lagoons are tested quarterly for biochemical oxygen demand, $\mathrm{pH}$, and total suspended solids. Annually, sewage lagoon pond waters are sampled for a suite of toxic chemicals. In 2004, quarterly and annual analyses of sewage influent and pond waters, respectively, both showed that all water measurements were within permit limits (see Section 4.2.3). The majority of samples had concentrations of toxic chemicals below detectable levels. The few toxic chemicals which were detectable were found at levels all less than 0.1 percent of their permit limits.

\section{Onsite Non-Radiological Drinking Water Quality}

NNSA/NSO operates a network of nine permitted wells that comprise three permitted public water systems on the NTS; these supply the potable water needs of NTS workers and visitors. In addition, three private water systems are maintained but are not regulated under state permit. NNSA/NSO hauls potable water for use in decontamination and sanitation for work locations at the NTS that are not part of a public water system. Monitoring results indicate that water samples from the three public water systems and from the potable water hauling trucks met the National Primary and Secondary Drinking Water Standards in 2004 (see Section 4.2.1). Samples from two of the water systems slightly exceeded a Secondary Standard for $\mathrm{pH}$. No monitoring of the private water systems was conducted.

\section{Non-Radiological Releases into Air and Water at the NLVF}

Like the NTS, the North Las Vegas Facility (NLVF) is regulated for the emission of criteria air pollutants and HAPs. Air quality operating permits are maintained for a variety of equipment at the NLVF. There are no monitoring requirements associated with these permits. The Clark County Health District requires submittal of an annual emissions inventory. The estimated quantities of criteria air pollutants and HAPs emitted at the NLVF in 2004 were minimal; they ranged from 0.0009 tons for HAPS to 0.679 tons for nitrous oxides (see Appendix B, Section B.1.3).

Water discharges at the NLVF are regulated by a permit with the City of North Las Vegas (CNLV) for sewer discharges and by temporary NPDES discharge permits with the state for groundwater discharges into the CNLV 
storm water drainage system. The NPDES permits were obtained for a groundwater characterization and dewatering project at the facility. Self-monitoring and reporting of the levels of non-radiological contaminants in sewage and industrial outfalls is conducted. In 2004, all water samples from NLVF outfalls and all sludge and liquid samples from the NLVF sand/oil interceptor had contaminant levels below established permit limits (see Appendix B, Section B.1.1). An unauthorized discharge of tritiated water occurred at the NLVF in 2004, as discussed below.

\section{Accidental or Unplanned Environmental Releases or Occurrences}

On the NTS, one environmental occurrence was reportable in 2004. About 75 gallons of oil leaked onto the soil from a 650-gallon oil holding tank mounted on a lubrication truck (see Section 2.11).

At the NLVF, one environmental occurrence was reportable in 2004 (see Appendix B, Section B.1.1.2). Parts of Building A-1 at the NLVF were contaminated with tritium by a previous contractor in 1995. During a pre-inspection and safety walkthrough in 2004, it was observed that a 5-gallon bucket of water was located near a floor drain and under the drainpipe of the air-handling unit in Room 4520 of Building A-1. The bucket was being used to catch condensate from the air handler. Tritiated water from the bucket had been emptied into the floor drain, constituting an unauthorized discharge to the city of North Las Vegas sewer system. Water samples from the bucket indicated a tritium concentration of $23,000 \pm 4,000(\mathrm{pCi} / \mathrm{L})$. NNSA/NSO reported the unauthorized discharge to the CNLV. Several actions required by the CNLV were taken to prevent future discharges and to document that the tritium was not detectable at the sewage outfall for the NLVF following each of the discharge incidents.

During preparation of this report, EPA informed the state that it was in direct violation of the Clean Water Act by issuing the temporary NPDES discharge permits for the NLVF that allowed discharge of pumped groundwater to the CNLV storm water drainage system. BN will implement one of several corrective action options presented by the state in order to comply with the EPA ruling and resolve this issue.

\section{Pollution Prevention/Waste Minimization Activities}

Decommissioned NTS buildings destined for disassembly and disposal were donated or sold for reuse. This waste minimization effort diverted approximately 27.9 metric tons (mtons) (30.8 tons) of waste from the NTS landfills in 2004. The Material Exchange Program reused 1.97 mtons (2.17 tons) of non-hazardous chemicals, equipment, and supplies. The BN Payroll Department converted to a paperless, electronic time keeping system. This new process eliminated the need for paper timecards and reduced the amount of paper waste by about 2.0 mtons (2.2 tons). Other significant waste reduction efforts continued in 2004 , such as selling scrap ferrous metal to a vendor for recycling (751.2 mtons [826.3 tons]) and offsite recycling of mixed paper and cardboard (518.7 mtons [570.6 tons]). Overall, a reduction of 114.8 mtons (126.3 tons) of hazardous wastes and 1,437.5 mtons (1,581.3 tons) of solid wastes were realized in 2004 (see Section 11.0).

\section{Overall Compliance with Environmental Laws, Regulations, and Policies}

NTS compliance status with over 100 applicable environmental laws, regulations, and policies are summarized in Section 2.0. The major categories of these drivers are listed below along with the 2004 percent compliance within each category. Where compliance for a category is not 100 percent, the non-compliance incidents are noted. 


\section{Environmental Compliance Summary for the NTS in 2004}

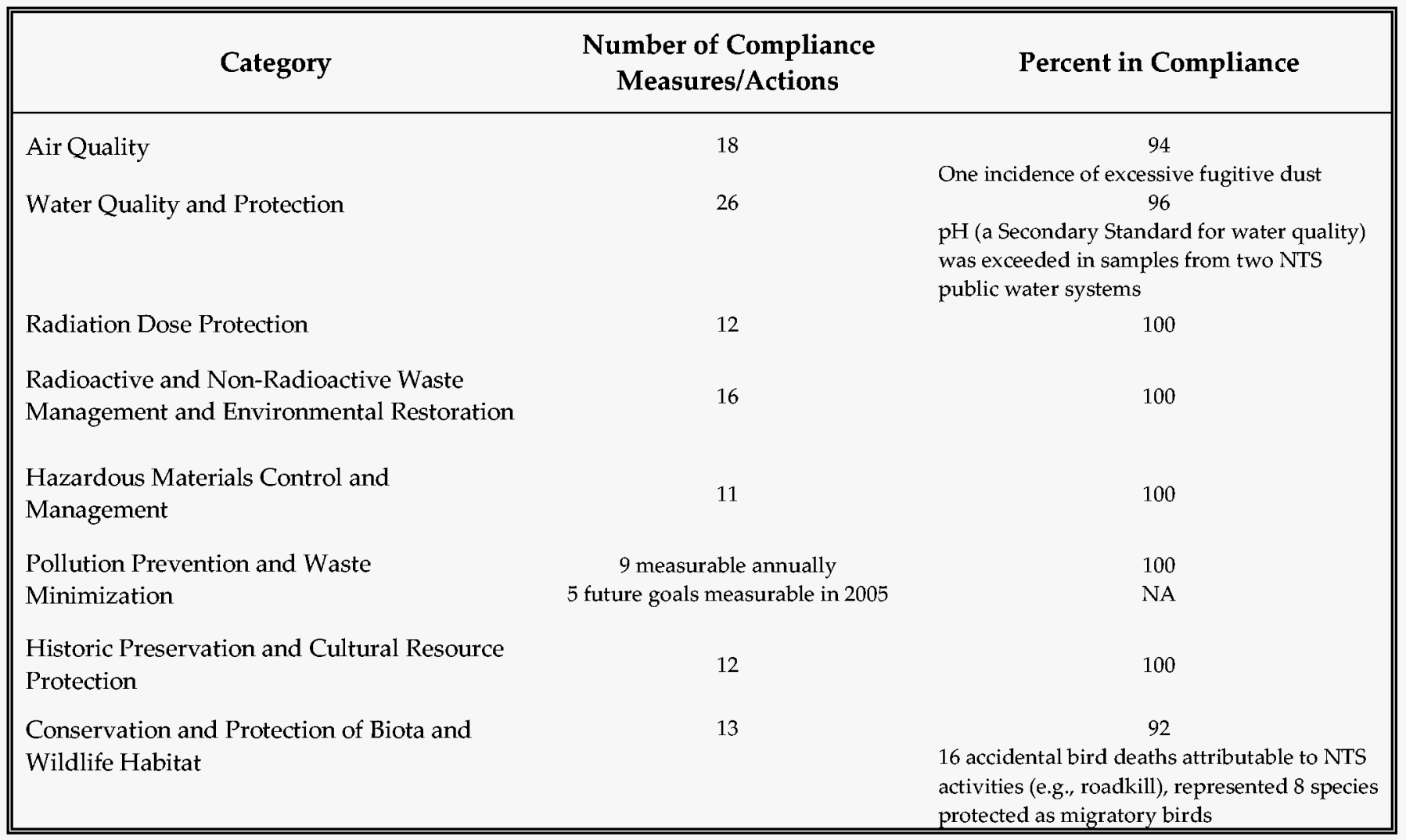




\subsection{Introduction}

\subsection{Site Location}

The U.S. Department of Energy, National Nuclear Security Administration Nevada Site Office (NNSA/NSO) directs the management and operation of the Nevada Test Site (NTS) which is located in Nye County in south-central Nevada (Figure 1-1). The southeast corner of the NTS is about 88 kilometers $(\mathrm{km})(55$ miles [mi]) northwest of the center of Las Vegas in Clark County. By highway, it is about $105 \mathrm{~km}$ (65 mi) from the center of Las Vegas to Mercury. Mercury, located at the southern end of the NTS, is the main base camp for worker housing and administrative operations for the NTS.

The NTS encompasses about 3,561 square kilometers $\left(\mathrm{km}^{2}\right)(1,375$ square miles [mi'] $)$. It varies from 46 to $56 \mathrm{~km}$ ( 28 to $35 \mathrm{mi}$ ) in width from west to east and from 64 to $88 \mathrm{~km}$ (40 to $55 \mathrm{mi}$ ) from north to south. The NTS is surrounded on all sides by federal lands. As shown in Figure 1-1, the NTS is bordered on the southwest corner by the Yucca Mountain Project Area, on the west and north by the Nevada Test and Training Range (NT'TR) (previously known as the Nellis Air Force Range), on the east by an area used by both the NTTR and the Desert National Wildlife Range (DNWR), and on the south by Bureau of Land Management lands. The combination of the NTTR and the NTS represents one of the larger unpopulated land areas in the United States, comprising some $14,200 \mathrm{~km}^{2}$ $\left(5,470 \mathrm{mi}^{2}\right)$.

\subsection{Environmental Setting}

The NTS is located in the southern part of the Great Basin, the northern-most sub-province of the Basin and Range Physiographic Province. The NTS terrain is typical of much of the Basin and Range Physiographic Province, characterized by generally north-south trending mountain ranges and intervening valleys. These mountain ranges and valleys, however, are modified on the NTS by very large volcanic calderas (Figure 1-2).

The principal valleys within the NTS are Frenchman Flat, Yucca Flat, and Jackass Flats (Figure 1-2). Both Yucca and Frenchman Flat are topographically closed and contain dry lake beds, or playas, at their lowest elevations. Jackass Flats is topographically open, and surface water from this basin flows off the NTS via the Fortymile Wash. The dominant highlands of the NTS are Pahute Mesa and Rainier Mesa (high volcanic plateaus), Timber Mountain (a resurgent dome of the Timber Mountain caldera complex), and Shoshone Mountain. In general, the slopes of the highland areas are steep and dissected, and the slopes in the lowland areas are gentle and less eroded. The lowest elevation on the NTS is $823 \mathrm{~m}(2,700 \mathrm{ft})$ in Jackass Flats in the southeast, and the highest elevation is $2,341 \mathrm{~m}$ $(7,680 \mathrm{ft})$ on Rainier Mesa in the north-central region.

The topography of the NTS has been altered by historic U.S. Department of Energy (DOE) actions, particularly underground nuclear testing. The principal effect of testing has been the creation of numerous collapse sinks ("craters") in Yucca Flat basin and a lesser number of "craters" on Pahute and Rainier Mesas. Shallow detonations were also performed during Project Plowshare to determine the potential uses of nuclear devices for large-scale excavation.

Figure 1-3 shows the general layout of the NTS, including the location of major facilities and the numbered operational areas of the NTS referred to in this report. The geographical areas previously used for nuclear testing are also shown in Figure 1-3.

The reader is directed to Appendix A where the geology, hydrology, climatology, ecology, and cultural resources of the site are described. 


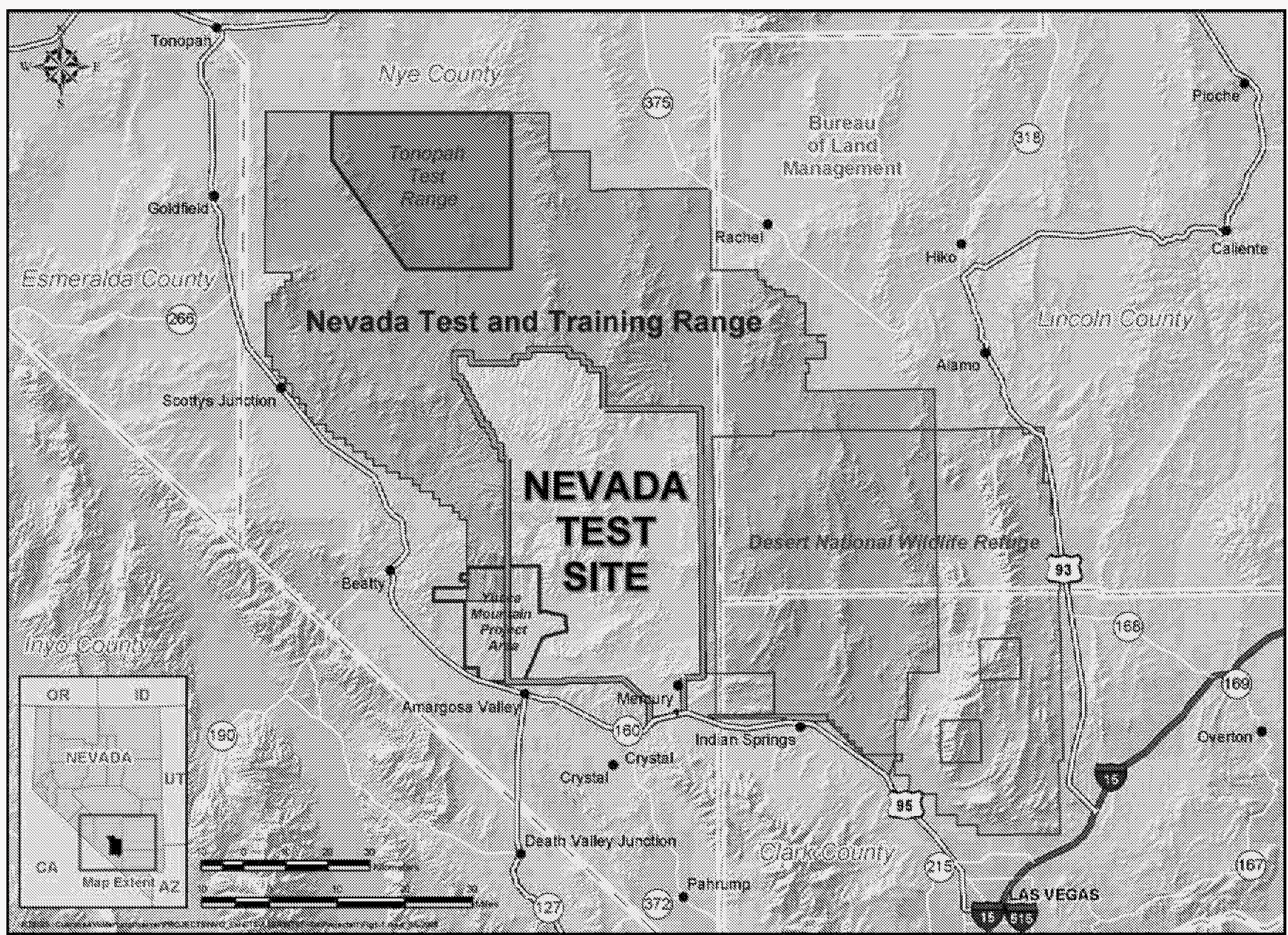

Figure 1-1. NTS vicinity map 


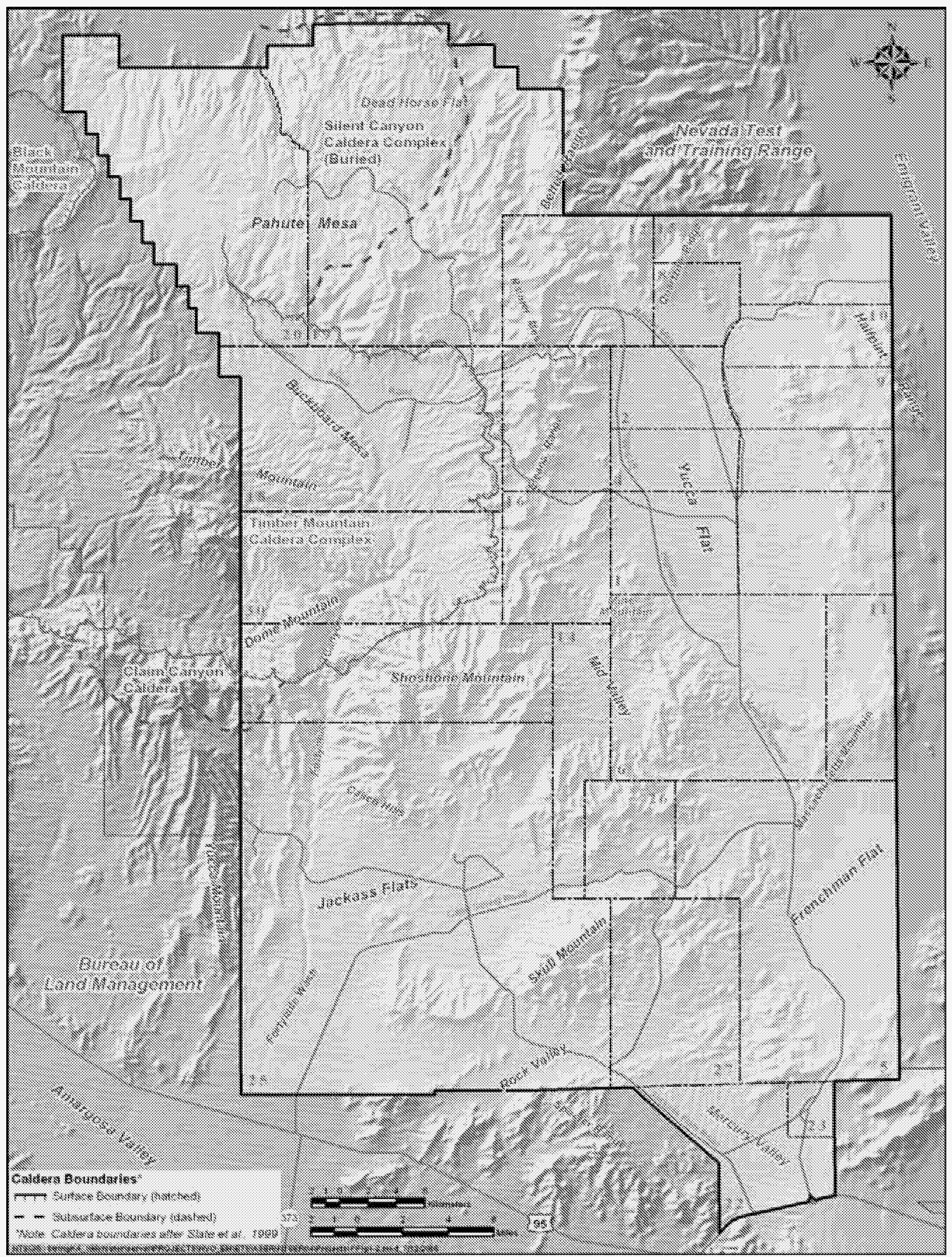

Figure 1-2. Major topographic features of the NTS 


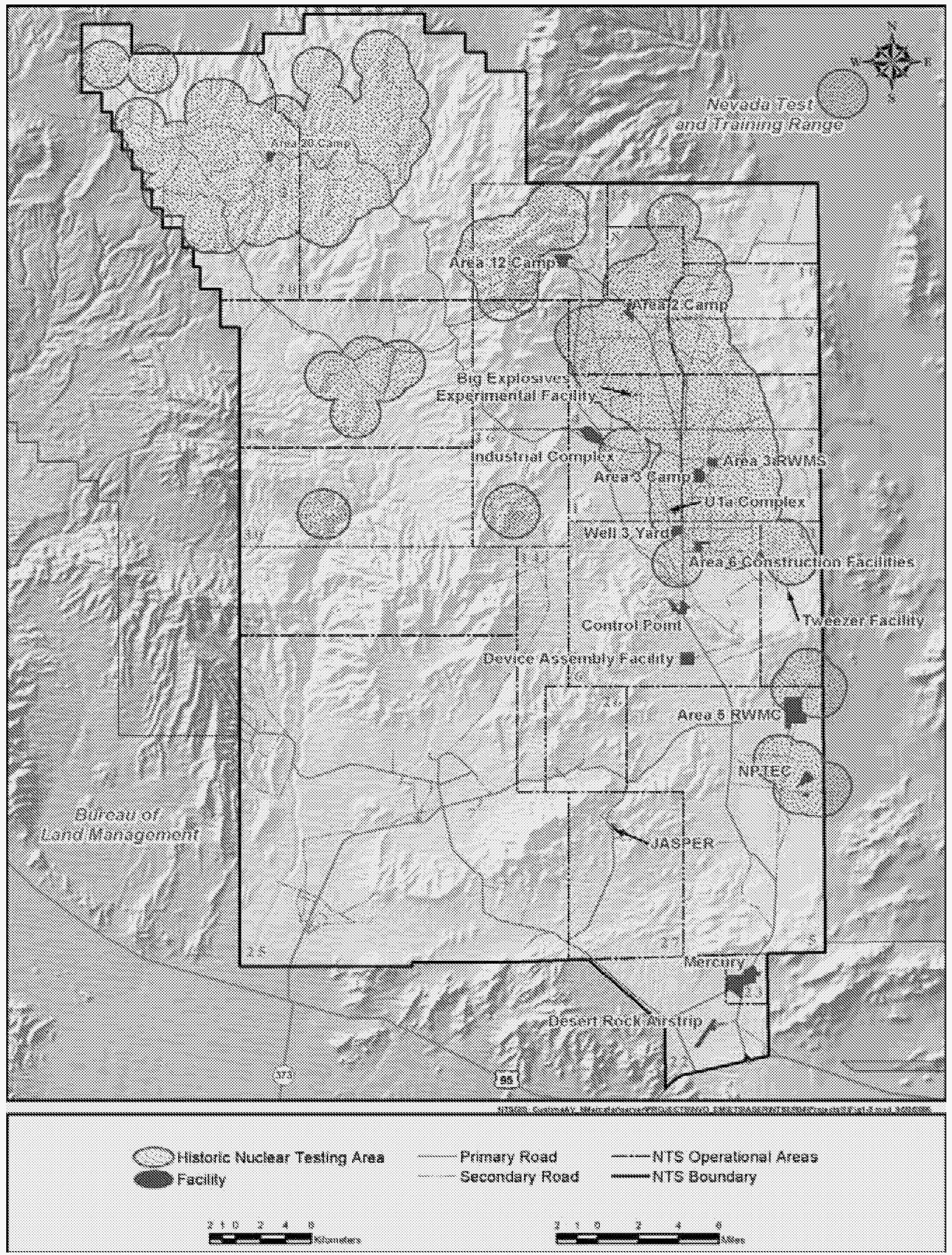

Figure 1-3. NTS operational areas, principal facilities, and past nuclear testing areas 


\subsection{Site History}

The history of the NTS, as well as its current missions, directs the focus and design of the environmental monitoring and surveillance activities on and near the site. Between 1940 and 1950, the area now known as the NTS was under the jurisdiction of Nellis Air Force Base and was part of the Nellis Bombing and Gunnery Range. The NTS was established in 1951 to be the primary location for testing the nation's nuclear explosive devices and supported nuclear testing from 1951 to 1992 . The NTS currently conducts only subcritical nuclear experiments.

Atmospheric Tests - Tests conducted through the 1950s were predominantly atmospheric tests. These tests involved a nuclear explosive device detonated while on the ground surface, on a steel tower, suspended from tethered balloons, dropped from an aircraft, or placed on a rocket. Several tests were categorized as "safety experiments", and "storage-transportation tests", involving the destruction of a nuclear device with non-nuclear explosives. Some of these tests resulted in the dispersion of plutonium in the test vicinity. One of these test areas lies just north of the NTS boundary at the south end of the NTTR, and four others involving storage-transportation are at the north end of the NTTR. These test areas have been monitored for radionuclides in the past $(1996-2000)$ in support of remediation projects, two of which were completed. The three remaining sites will be monitored again once restoration of these sites begins. All nuclear device tests are listed in United States Nuclear Tests, July 1945 through September 1992 (DOE, 2000a).

Underground Tests - The first underground test, a cratering test, was conducted in 1951. The first totally-contained underground test was in 1957. Testing was discontinued during a moratorium that began October 31, 1958, but was resumed in September 1961 after tests by the Union of Soviet Socialist Republics began. Since late 1962, nearly all tests have been conducted in sealed vertical shafts drilled into Yucca Flat and Pahute Mesa or in horizontal tunnels mined into Rainier Mesa. From 1951 to 1992, a total of 828 underground nuclear tests were conducted at the NTS. Approximately one third of these tests were detonated near or below the water table; this has resulted in the contamination of groundwater in some areas. In 1996, DOE, U.S. Department of Defense (DoD), and the state of Nevada entered into a Federal Facilities Agreement and Consent Order (FFACO) which established Corrective Action Units (CAUs) on the NTS that delineated and defined areas of concern for groundwater contamination.

Cratering Tests - Five earth-cratering (shallow-burial) tests were conducted over the period of 1962 through 1968 as part of the Plowshare Program that explored peaceful uses of nuclear explosives. The first and highest yield Plowshare crater test, Sedan (PHS, 1963) was detonated at the northern end of Yucca Flat on the NTS. The second highest yield crater test was Schooner, located in the northwest corner of the NTS. From these tests, mixed fission products, tritium, and plutonium were entrained in the soil ejected from the craters and deposited on the ground surrounding the craters.

Other Tests - Other nuclear-related tests and experiments at the NTS have included the Bare Reactor Experiment Nevada (BREN) series in the 1960s conducted in Area 25. These tests were performed with a 14 million electron volt $(\mathrm{MeV})$ neutron generator mounted on a 465-meter (1,530-feet) steel tower used to conduct neutron and gamma-ray interaction studies on various materials. From 1959 through 1973, a series of open-air nuclear reactor, nuclear engine, and nuclear furnace tests were conducted in Area 25, and a series of tests with a nuclear ramjet engine were conducted in Area 26. Erosion of metal cladding on the reactor fuel released some fuel particles that caused negligible deposition of radionuclides on the ground. Most of the radiation released from these tests was gaseous in the form of radioiodines, radio-xenons, radio-kryptons.

\subsection{Site Mission}

NNSA/NSO directs the management and operation of the NTS and seven satellite sites across the nation. Los Alamos National Laboratory (LANL), Lawrence Livermore National Laboratory (LLNL), and Sandia National Laboratories (SNL) are the principal organizations that sponsor and implement the nuclear weapons programs at the NTS. Bechtel Nevada (BN) is the Management and Operations ( $M \& O$ ) contractor who is accountable for the successful execution of work and ensuring that work is performed in compliance with environmental regulations. The seven satellite sites of the NTS include the North Las Vegas Facility (NLVF), Cheyenne Las Vegas Facility (CLVF), Remote Sensing Laboratory (RSL) - Nellis, RSL - Andrews, Livermore Operations, Los Alamos Operations, and 
Special Technologies Laboratory. These sites all provide support to enhance the NTS as a site for weapons experimentation and nuclear test readiness. This report addresses environmental monitoring and compliance only at the NTS and its three Nevada satellite sites: NLVF, CLVF, and RSL-Nellis (see Appendix B). The three major NTS programs include: (1) Stockpile Stewardship, (2) National Security Response Program and Operations, and (3) Environmental Management.

\section{NTS Program Missions}

Stockpile Stewardship - The primary mission of this program is to conduct high-hazard operations in support of defense-related nuclear and national security experiments and to maintain the capabilities to resume underground nuclear weapons testing, if directed.

National Security Response Program and Operations - The goal of this program is to provide support facilities, training facilities, and capabilities for government agencies involved in counterterrorism activities, emergency response, first responders, national security technology development, and nonproliferation technology development.

Environmental Management - This program includes Waste Management and Environmental Restoration. The goals of this program are to manage and safely dispose of low-level waste received from DOE and DoD-approved facilities throughout the United States and mixed low-level waste generated in Nevada by NNSA/NSO operations, safely manage and characterize for offsite disposal hazardous and transuranic wastes, characterize and remediate the environmental legacy of nuclear weapons and other testing at the NTS and at offsite locations, and develop and deploy technologies that enhance environmental restoration.

\subsection{Primary Operations and Activities}

NTS activities in 2004 continue to be diverse, with the primary role being to help ensure that the existing United States stockpile of nuclear weapons remains safe and reliable. Facilities that support this mission include the U1a Facility, Big Explosives Experimental Facility (BEEF), Device Assembly Facility (DAF), and Joint Actinide Shock Physics Experimental Research (JASPER) Facility. Other NTS activities include demilitarization activities; controlled spills of hazardous material at the Non-Proliferation Test and Evaluation Complex (NPTEC) (formerly known as the Hazardous Materials Spill Center); remediation of industrial sites; processing of waste destined for the Waste Isolation Pilot Plant in Carlsbad, New Mexico; disposal of radioactive and mixed waste; and environmental research. In addition, there are continued efforts to bring other business to the NTS, like aerospace and alternative energy technologies and support of U.S. Department of Homeland Security National Center for Combating Terrorism work.

\subsection{Demography}

The population of the area surrounding the NTS is predominantly rural. The population estimates for Nevada communities has been estimated by the Nevada State Demographer Office (NSDO) up through July 1, 2004 (Hardcastle, 2005). The annual population estimate for Nevada counties, cities, and unincorporated towns is 2,410,768, with all but 695,431 residing in Clark County. The total population estimate for Nye County is 38,131 and includes the communities of Amargosa Valley (1,211), Beatty (981), Gabbs (316), Manhattan (128), Pahrump (30,465), Round Mountain (767), and Tonopah (2,341). The largest of the Nye County communities is Pahrump Valley, which is approximately $50 \mathrm{mi}(80 \mathrm{~km})$ south of the NTS Control Point facility, which is near the center of the NTS (see Figure A-2). Neighboring Lincoln County to the east of the NTS includes a few small communities including Alamo (441), Caliente (1,014), Panaca (552), and Pioche (669). Neighboring Clark County is the major population center of Nevada and has an estimated total population of 1,715,337. 
The Mojave Desert of California, which includes Death Valley National Park, lies along the southwestern border of Nevada. This area is still predominantly nural; however, tourism at Death Valley National Park swells the population to more than 5,000 on any particular day during holiday periods during mild weather.

The extreme southwestern region of Utah is more developed than the adjacent portion of Nevada. The population estimates for Utah communities are based on the 2000 Census data and were obtained from the U.S. Census Bureau. The largest community is St. George, located $220 \mathrm{~km}(137 \mathrm{mi})$ east of the NTS, with a population of 49,663. The next largest town, Cedar City, is located $280 \mathrm{~km}(174 \mathrm{mi})$ east-northeast of the NTS and has a population of $20,527$.

The extreme northwestern region of Arizona is mostly rangeland, except for that portion in the Lake Mead recreation area. In addition, several small communities lie along the Colorado River. The largest towns in the area are Bullhead City, $165 \mathrm{~km}$ (103 mi) south-southeast of the NTS, with an estimated population of 37,325, and Kingman, located $280 \mathrm{~km}(174 \mathrm{mi}$ ) southeast of the NTS, with an estimated population of 24,600 (July 1, 2004 population estimates, Arizona Department of Economic Security, 2004).

The offsite population density within an $80-\mathrm{km}$ radius of all emission sources of radioactivity on the NTS is about 1.0 persons $/ \mathrm{km}^{2}$ (2.6 persons $\left./ \mathrm{mi}^{2}\right)$. In comparison, the 48 contiguous states have a population density of about 36 persons $/ \mathrm{km}^{2}\left(94\right.$ persons $\left./ \mathrm{mi}^{2}\right)$ (U.S. Census Bureau, 2005). 


\section{THIS PAGE INTENTIONALLY LEFT BLANK}




\subsection{Compliance Summary}

Environmental regulations pertinent to operations on the Nevada Test Site (NTS) and its Nevada satellite facilities (North Las Vegas Facility [NLVF], Cheyenne Las Vegas Facility [CLVF], and Remote Sensing Laboratory [RSL]Nellis) are listed in this Compliance Summary. They include federal and state laws, state permit requirements, Executive Orders (EOs), DOE Orders, and state agreements. 'They dictate how the U. S. Department of Energy, National Nuclear Security Administration Nevada Site Office (NNSA/NSO) conducts operations on and off the NTS to ensure the protection of the environment and the public. The regulations are grouped by topic. A compliance status table is presented for each topical group of regulations. Each table lists those measures or actions which are tracked or performed annually to ensure compliance with a regulation. A description of the field monitoring efforts, actions, and results which support the data in each table can be found in subsequent sections of this document, as noted in the "Reference Section" column of each table. Non-compliance incidents or compliance issues, if any, are included in the topical subsections along with a listing of compliance reports generated during the reporting year. The last table presented in this section is a list of all NNSA/NSO environmental permits for the NTS and its satellite facilities for the year 2004 .

\subsection{Air Quality}

Clean Air Act (CAA), National Emission Standards for Hazardous Air Pollutants (NESHAP) - Under Title III of the CAA, NESHAP was established to control those pollutants that might reasonably be anticipated to result in either an increase in mortality or an increase in serious irreversible or incapacitating but reversible illness. Industry-wide national emissions standards were developed for 22 of the 189 designated hazardous air pollutants (HAPs). Radionuclides and asbestos were among the $22 \mathrm{HAPs}$ for which standards were established. These standards are promulgated through Title 40 of the Code of Federal Regulations (CFR), Part 61, in Subparts $\mathrm{H}$ and M, respectively. Under Subpart H, NESHAP establishes a radiation dose limit for individuals of the general public. Subpart $\mathrm{M}$ addresses protection of the public from asbestos. Both subparts define the methods to use in determining compliance, recordkeeping, reporting, and in determining whether federal approval is required prior to the construction of new facilities or the modification of existing facilities. NESHAP compliance activities at the NTS are limited to radionuclide monitoring and reporting and notification of asbestos abatement. No NESHAP compliance activities are required at the Nevada satellite facilities.

CAA, National Ambient Air Quality Standards (NAAQS) - Title I of the CAA established the NAAQS to limit levels of pollutants in the air for six "criteria" pollutants: sulfur dioxide, nitrogen oxides, carbon monoxide, ozone, lead, and particulate matter. Title V of the CAA authorizes the states to implement permit programs in order to regulate emissions of the criteria pollutants. At the NTS there is one main permit that regulates operations and emissions from aggregate-producing facilities, fuel-burning equipment, fuel storage, project-specific activities associated with the Non-Proliferation Test and Evaluation Complex (NPTEC) (formerly the Hazardous Materials Spill Center), Test Cell C Facility, and the Tactical Demilitarization Development Project (TaDD). Detonations conducted at the Big Explosives Experimental Facility (BEEF) and the Explosives Ordnance Disposal Unit (EODU), which heretofore did not require permitting, are now included in the permit. The NTS also has a temporary air permit for a portable screening and crushing plant in Area 6. Nevada air quality permits specify emission limits for criteria pollutants (except ozone and lead) that are based on published emissions values for other similar industries and on operational data specific to the NTS. Lead is considered a HAP as well as a criteria pollutant. Emissions from lead are reported as part of the total HAPs emissions rather than as a separate criteria pollutant. Lead emissions above a specified threshold are also reported under Section 313 of the Emergency Planning and Community Right-to-Know Act (see Section 2.5). Quantities of NAAQS and HAPS emissions from operations at the NTS are calculated and submitted each year to the state of Nevada. The NTS air permit also specifies recordkeeping and reporting requirements, visible emissions (opacity) limits for equipment or facilities, opacity field monitoring 
requirements, and certification requirements for personnel conducting opacity monitoring. The permit also grants the state access to the NTS to conduct inspections of permitted facilities.

State of Nevada regulations prohibit the open burning of combustible refuse and other materials unless specifically exempted by an authorized variance (Nevada Administrative Code 445B.122). At the NTS, Open Burn Variances are routinely obtained for fire extinguisher training and various emergency management exercises.

The NTS satellite facilities discussed in Appendix B operate under air quality permits that require the reporting of estimated annual emissions of criteria pollutants.

CAA, New Source Performance Standards (NSPS) - The NSPS were established by Title I of the CAA to set minimum nationwide emission limitations of regulated air pollutants (HAPs and criteria pollutants mentioned above) and for various industrial categories of facilities. The state of Nevada has adopted the NSPS and regulates emissions from subject facilities through state law (NRS 445B as codified in NAC 445B). At the NTS, some of the screens and conveyor belts that were manufactured after August 1981 are subject to NSPS under the category of Nonmetallic Mineral Processing Plants. The NSPS imposes more stringent standards, including a reduced allowance of visible emissions (opacity) than under NAAQS. NSPS compliance activities on the NTS are reported to the state of Nevada. The NTS satellite facilities discussed in Appendix B operate under air quality permits that require the reporting of estimated annual emissions of HAPs.

CAA, Stratospheric Ozone Protection - Title VI (Section 608) of the CAA establishes production limits and a schedule for the phase-out of ozone-depleting substances (ODS). ODS are defined as those substances that are known or could reasonably be anticipated to cause or contribute to stratospheric ozone depletion. Under Section 608, the U.S. Environmental Protection Agency (EPA) has established regulations through 40 CFR Part 82 that include: (1) maximizing recycling of ozone-depleting compounds during servicing and disposal of air conditioning and refrigeration equipment, (2) establishing requirements for recycling and recovery equipment, technicians and reclaimers, (3) requiring the repair of substantial leaks in certain air conditioning and refrigeration equipment, and (4) establishing safe disposal requirements. While there are no reporting requirements for ODS, recordkeeping to document the usage of ODS and technician certification is required. Under Section 608, the EPA may conduct random inspections to determine compliance.

At the NTS, refrigerants containing ODS are mainly used in air conditioning units in vehicles, buildings, refrigerators, water fountains, vending machines, and laboratory equipment. Halon 1211 and 1301, now classified as ODS, have been used in the past in fire extinguishers. Self-assessments are conducted periodically to document adherence to Title VI of the CAA.

Other NTS Air Quality Permit Requirements - Under Title V, Part 70 of the CAA amendments, all owners or operators of Part 70 sources must pay annual fees to the state. Any source which has the potential to emit 45.4 metric tons (mtons) (50 tons) or more of any regulated air pollutant, except carbon monoxide, must pay an annual fee of $\$ 3,000$. Any source that has the potential to emit less than $22.7 \mathrm{mtons}$ ( 25 tons) per year of any regulated air pollutant, except carbon monoxide, must pay an annual fee of $\$ 250$. NTS operations are subject to these fees. In addition to permit fees, NNSA/NSO must allow the state of Nevada Bureau of Air Pollution Control to conduct inspections of NTS facilities and operations that are regulated by state air quality permits.

Section VII of the NTS Class II Air Quality Operating Permit, No. AP9711-0549.01 Surface Area Disturbance Conditions requires implementation of an ongoing program to control fugitive dust using the best practicable methods.

\subsubsection{Compliance Issues}

During the summer of 2004, NNSA/NSO personnel observed an excess of fugitive dust resulting from an operation at the Area 5 Radioactive Waste Management Complex. NNSA/NSO determined that Bechtel Nevada (BN) was failing to monitor operations sufficiently to prevent excessive fugitive dust. In response, BN Environmental Services 
finalized a fugitive dust control policy in November (Organization Instruction, OI-0442.002 Fugitive Dust Monitoring). This policy establishes periodic monitoring of dust-producing activities and operations.

\subsubsection{Compliance Reports}

The following reports were generated for NTS operations in 2004 in compliance with air quality regulations:

- National Emissions Standards for Hazardous Air Pollutants, Calendar Year 2004 (submitted to EPA Region IX)

- Annual Asbestos Abatement Notification Form, submitted to EPA Region IX

- Calendar Year 2004 Actual Production/Emissions Reporting Form, submitted to the Nevada Division of Environmental Protection

- Quarterly Class II Air Quality Report, submitted to the Nevada Division of Environmental Protection

- NPTEC Pre-test and Post-test Reports, submitted to the Nevada Division of Environmental Protection

The following reports were generated for operations at NTS satellite facilities in 2004 in compliance with air quality regulations:

- Clark County Air Emission Inventory for North Las Vegas Facility, submitted to the Clark County Department of Air Quality and Environmental Management

- Clark. County Air Emissions Inventory for Remote Sensing Laboratory, submitted to the Clark County Department of Air Quality and Environmental Management

\subsubsection{Compliance Status}

See Table 2-1 for a summary of how NNSA/NSO complied with air quality and protection regulations at the NTS and its satellite facilities in 2004. 


\begin{tabular}{|c|c|c|c|}
\hline Compliance Measure/Actions & Compliance Limit & Compliance Status - 2004 & $\begin{array}{c}\text { Section } \\
\text { Reference }\end{array}$ \\
\hline \multicolumn{4}{|l|}{ Clean Air Act - NESHAP } \\
\hline Annual dose equivalent from all radioactive air emissions & $\begin{array}{c}10 \mathrm{mrem} / \mathrm{yr}^{(\mathrm{b})} \\
(0.1 \mathrm{mSv} / \mathrm{yr})\end{array}$ & $\begin{array}{l}\text { Compliant - } 0.12 \mathrm{mrem} / \mathrm{yr}^{(\mathrm{b})} \\
\quad(0.0012 \mathrm{mSv} / \mathrm{yr})\end{array}$ & $3.1 .5 ; 8.1 .3$ \\
\hline $\begin{array}{l}\text { Notify EPA Region IX if the number of linear or square feet (ft) of asbestos to } \\
\text { be removed from a facility exceeds limit }\end{array}$ & 260 linear $\mathrm{ft}$ or $160 \mathrm{ft}^{2(\mathrm{c})}$ & Compliant & 3.2 .7 \\
\hline $\begin{array}{l}\text { Maintain asbestos abatement plans, data records, and activity/maintenance } \\
\text { records }\end{array}$ & For up to 25 or 75 years & Compliant & 3.2 .7 \\
\hline Submit quarterly reports of calculated emissions to state of Nevada & Due 30 days after end of quarter & Compliant & 3.2 .1 \\
\hline Submit annual report of calculated emissions to state of Nevada & Due March 1 & Compliant & 3.2 .1 \\
\hline $\begin{array}{l}\text { Number of gallons of fuel used, hours of operation, and rate of } \\
\text { aggregate/concrete production by permitted equipment/facility }\end{array}$ & Limit varies(d) & Compliant & 3.22 \\
\hline $\begin{array}{l}\text { Tons of emissions of each criteria pollutant produced by permitted } \\
\text { equipment/facility based on calculations }\end{array}$ & PTE varies ${ }^{(e)}$ & Compliant & $\begin{array}{l}3.2 .1 ; \\
\text { Table 3-12 }\end{array}$ \\
\hline $\begin{array}{l}\text { Submit test plans/final analysis reports for tests at NPTEC facilities and } \\
\text { annual report of all chemicals released during the year to the state }\end{array}$ & Annual report due March 1 & $\begin{array}{l}\text { Compliant } \\
5 \text { tests conducted }\end{array}$ & $\begin{array}{c}3.24 ; \\
\text { Table 3-14 }\end{array}$ \\
\hline $\begin{array}{l}\text { Estimated quantities of criteria air pollutants and HAPs emitted annually at } \\
\text { the NLVF and the RSL submitted in annual emissions inventory reports to } \\
\text { Clark County Health District }\end{array}$ & $\begin{array}{c}\text { No emissions limits, } \\
\text { Emissions Inventories due } \\
\text { March } 31\end{array}$ & Compliant & $\begin{array}{l}\text { B.1.3; } \\
\text { Table B-4; } \\
\text { B.3.2; } \\
\text { Table B-8 }\end{array}$ \\
\hline \multicolumn{4}{|l|}{ Clean Air Act - NSPS } \\
\hline Conduct opacity readings from permitted equipment/facility & Quarterly & Compliant & 3.2 .3 \\
\hline Percent opacity of emissions from permitted equipment/facility & $10 \%$ & $\begin{array}{l}\text { Compliant } \\
<10 \% \text { for } 1 \text { facility }\end{array}$ & 3.2 .3 \\
\hline
\end{tabular}


Table 2-1. (continued)

Clean Air Act - Stratospheric Ozone Protection

Maintain ODS technician certification records, approvals for ODS-containing equipment recycling/recovery, and applicable equipment servicing records

Generic Nevada Air Quality Permit Regulations

Implement program to control fugitive dust for land disturbing activities

Non-compliant

Allow Nevada Bureau of Air Pollution Control access to conduct inspections of facilities and operations regulated by state air permits

NA

$$
\begin{gathered}
\text { One incidence of excessive } \\
\text { fugitive dust } \\
\text { Compliant }
\end{gathered}
$$

Inspection conducted in May

(a) The section(s) within this document that describe how compliance summary data were collected

(b) $\mathrm{mrem} / \mathrm{yr}=$ millirem per year; $\mathrm{mSv} / \mathrm{yr}=$ millisievert per year

(c) 260 linear $\mathrm{ft}$ or $160 \mathrm{ft}^{2}=79.3$ linear meters (m) or $14.9 \mathrm{~m}^{2}$

(d) Compliance limit is specific for each piece of permitted equipment/facility

(e) Potential to emit (PTE) = the quantities of criteria pollutants that each facility/piece of equipment would emit annually if it were operated for the maximum number of hours specified in the state air permit

(f) Not applicable 


\subsection{Water Quality and Protection}

Clean Water Act (CWA) -Prohibits the discharge of pollutants from point sources to waters of the U.S. without a National Pollutant Discharge Elimination System (NPDES) permit. The CWA also gives the EPA, or the approved state environmental control agency, the authority to implement pollution control programs. The CWA also sets water quality standards for all contaminants in surface waters. At the NTS, applicable CWA regulations are followed through compliance with permits issued by the Nevada Division of Environmental Protection (NDEP) and the Nevada State Health Division, Bureau of Health Protection Services (BHPS) for wastewater discharges and disposal of wastewater from facilities. On the NTS, no wastewater discharges are released into any waters of the U.S. Therefore, NTS operations do not require any NPDES permits. Three NPDES permits have been issued to NNSA/NSO for the discharge of pumped groundwater at the NLVF into the North Las Vegas storm water drainage system during the conduct of a groundwater characterization study (see Appendix B).

Safe Drinking Water Act (SDWA) - Protects the quality of drinking water in the U.S. This law focuses on all waters actually or potentially designed for drinking use, whether from above-ground or underground sources. It authorizes the EPA to establish safe standards of purity and requires all owners or operators of public water systems to comply with National Primary Drinking Water Standards (health-related standards). State governments, which assume this power from the EPA, also set Secondary Standards which are related to taste, odor, and visual aspects of drinking water. Nevada state law pertaining to public water systems (NAC 445A) ensures that such water systems meet the EPA water quality standards specified under the SDWA.

Nevada Administrative Code (NAC) 445A - Water Controls (Public Water Systems) - Enforces the SDWA requirements and sets standards for permitting, design, construction, operation, maintenance, certification of operators, and water quality of public water systems (PWSs). The NTS has three PWSs and two potable water hauler trucks which BHPS regulates through the issuance of permits. Although the SDWA sets drinking water standards for radionuclides, the state of Nevada does not require radionuclide monitoring of drinking water on the NTS because the NTS does not have a "community water system" (i.e., a PWS having at least 15 service connections and used by year-round residents). However, all potable water supply wells are monitored on the NTS for radionuclides in compliance with DOE Order 5400.5 Radiation Protection of the Public and the Environment (see Section 2.3).

NAC 444 and 445A - Water Controls (Water Pollution Control) - Regulates the collection, treatment, and disposal of wastewater and sewage at the NTS. The requirements of this state regulation are issued in permits for E Tunnel effluent waters, sewage lagoons, septic tanks, and septic hauler contractors and pumpers. Perched groundwater which seeps out of E Tunnel in Area 12 is contained and monitored annually for radiological contaminants and quarterly for non-radiological contaminants as required under an NDEP permit issued to the Defense Threat Reduction Agency (DTRA). NNSA/NSO holds a general permit issued by NDEP covering two active and nine inactive sewage lagoon systems (see Table 2-12). Water quality and toxicity of the active sewage lagoons are monitored quarterly and annually, respectively, to meet permit requirements. The 19 septic systems on the NTS each process less than 5,000 gallons per day (gal/d) (18,927 liters/day), therefore they are not regulated by NDEP. The BHPS regulates the NTS septic systems as commercial individual systems which treat domestic sewage only in quantities less than $5,000 \mathrm{gal} / \mathrm{d}$. The BHPS does not require collection or analysis of sewage samples from these septic systems. The BHPS also regulates the permits that NNSA/NSO holds for the four septic tank pumpers, one septic tanker, and one septic tank pumping contractor.

Discharges of sewage and industrial wastewater from the NLVF are required to meet permit limits set by the City of North Las Vegas. Discharges of wastewater from the RSL are required to meet permit limits set by the Clark County Water Reclamation District.

NAC 534 - Nevada Division of Water Resources Regulations for Water Well and Related Drilling Regulates the drilling and construction of new wells and the reworking of existing wells in order to prevent the waste of underground waters and their pollution or contamination. Two site operations that are affected by this state regulation are the Underground Test Area (UGTA) Project and the Borehole Management Project. New water wells are drilled for ongoing UGTA investigations of site-specific hydrogeologic characteristics, underground source terms, 
and contaminant movement through groundwater. Over 1,100 existing boreholes on the NTS are being plugged according to these regulations, under the Borehole Management Project.

\subsubsection{Out-of-Compliance Incidents}

Septic System, Area 27, Able Compound Permit NY-1087 - On Monday, March 1, 2004, approximately 30 gallons of raw sewage was accidentally released onto the ground from the manhole above the Able Compound septic tank. The manhole was pumped and the sewer line was jetted to clear the obstruction (roots and sediment). No fines or penalties were incurred from this discharge.

Septic System, Area 25, Reactor Control Point Permit NY-1086 - On Thursday, April 8, 2004, at Building 25-3102, a valve was missing from a cooling radiator. While the laborers were cutting water pipes on the inside of the building, water started to flow from the radiator and approximately 2.8 liters of water made it into a drain about 10 feet away. No fines or penalties were incurred from this discharge.

\subsubsection{Compliance Reports}

The following reports were generated for NTS operations in 2004 in compliance with water quality regulations:

- Quarterly Monitoring Report for Nevada Test Site Sewage Lagoons, submitted April 13, July 13, and October 18, 2004, and January 13, 2005 to NDEP (in compliance with permit GNEV93001)

- Results of water quality analyses for PWSs were sent to the state throughout the year as they were obtained from the laboratory.

- Water Pollution Control Permit NEV 96021, Quarterly Monitoring Report (for E Tunnel effluent monitoring), submitted April 20, July 20, and October 19, 2004, and January 24, 2005 to NDEP

- Water Pollution Control Permit NEV 96021 Quarterly Monitoring Report and Annual Summary Report for E Tunnel Waste Water Disposal System (DTRA, 2004)

The following reports were generated for operations at the NTS satellite facilities in 2004 in compliance with water quality regulations:

- Self-Monitoring Report for the National Nuclear Security Administration's North Las Vegas Facility: Permit VEH-112, submitted October 25, 2004 to the City of North Las Vegas

- Quarterly reports titled Remote Sensing Laboratory Self Monitoring Report-Permit No. CCWRD-080, submitted March 3, May 6, September 7, and December 1, 2004 to the Clark County Water Reclamation District

- Two additional monitoring reports titled Remote Sensing Laboratory Additional Monitoring Reports - Permit No. $C C W R D-080$ were submitted February 4 and July 1, 20

- Reports of groundwater discharge volumes for NLVF temporary NPDES permits TNEV2003461, TNEV2004348, and 'TNEV2004364 04, submitted each month to NDEP

\subsubsection{Compliance Status}

See Table 2-2 for a summary of how NNSA/NSO complied with water quality and protection regulations at the NTS and its satellite facilities in 2004. 
Table 2-2. NTS compliance status with applicable water quality and protection regulations

\begin{tabular}{|c|c|c|c|}
\hline Compliance Measure/Action & Compliance Limit & Compliance Status - 2004 & $\begin{array}{c}\text { Section } \\
\text { Reference }^{(a)}\end{array}$ \\
\hline \multicolumn{4}{|l|}{$\begin{array}{l}\text { Safe Drinking Water Act and Nevada Water Controls (NAC 445A - Water } \\
\text { Controls - Public Water Systems) }\end{array}$} \\
\hline Number of water samples containing coliform bacteria & 1 per month & 0 & 4.2.1.1; Table 4-10 \\
\hline Concentration of nitrates in all PWSs (in milligrams per liter $[\mathrm{mg} / \mathrm{L}]$ ) & $10 \mathrm{mg} / \mathrm{L}$ & $\mathrm{ND}^{(\mathrm{b})}-4.3 \mathrm{mg} / \mathrm{L}$ & 4.2.1.1; Table 4-10 \\
\hline Concentration of organic contaminants in all PWSs & Limit varies(c) & Compliant & 4.2.1.1; Table 4-10 \\
\hline Concentration of Phase V inorganic contaminants in all PWSs & Limit varies & Compliant & 4.2.1.1; Table 4-10 \\
\hline Concentration of disinfection byproducts in all PWSs & $0.06-0.08 \mathrm{mg} / \mathrm{L}$ & $0.0011-0.0066$ & 4.2.1.1; Table 4-10 \\
\hline Concentration of secondary standards in all PWSs & Limit varies & $\begin{array}{l}\text { Compliant for all standards } \\
\text { except pH from Area } 23 \text { and } 6 \\
\text { PWS and Area } 25 \text { PWS }\end{array}$ & 4.2.1.1; Table $4-10$ \\
\hline Concentration of lead and copper in Area 12 PWS & Limit varies & Compliant & 4.2.1.1; Table 4-10 \\
\hline Concentration of fluoride in Area 25 PWS & $4.0 \mathrm{mg} / \mathrm{L}$ & $1.8-2.4 \mathrm{mg} / \mathrm{L}$ & 4.2.1.1; Table 4-10 \\
\hline $\begin{array}{l}\text { Adhere to design, construction, maintenance, and operation regulations } \\
\text { specified by permits }\end{array}$ & $\mathrm{NA}^{(\mathrm{d})}$ & Compliant & \\
\hline Allow BHPS access to conduct inspections of PWS and water hauling trucks & NA & Compliant & 4.2 .1 .2 \\
\hline \multicolumn{4}{|l|}{$\begin{array}{l}\text { Clean Water Act - National Pollutant Discharge Elimination System/State } \\
\text { Pollutant Discharge Elimination System Permits }\end{array}$} \\
\hline $\begin{array}{l}\text { Measure and report volume of pumped groundwater discharged at the } \\
\text { NLVF }\end{array}$ & NA & Compliant & $\begin{array}{l}\text { Appendix B, } \\
\text { B.1.1.3; Table B-3 }\end{array}$ \\
\hline \multicolumn{4}{|l|}{$\begin{array}{l}\text { Clean Water Act and Nevada Water Pollution Controls - Sewage Disposal } \\
\text { (NAC } 444 \text { - Sewage Disposal) }\end{array}$} \\
\hline $\begin{array}{l}\text { Adhere to all design/construction/operation requirements for new systems } \\
\text { and those specified in } 16 \text { septic system permits, } 5 \text { septic tank pumper } \\
\text { permits, and } 1 \text { septic tank pumping contractor permit }\end{array}$ & NA & Compliant & 4.2 .3 \\
\hline \multicolumn{4}{|l|}{$\begin{array}{l}\text { Clean Water Act and Nevada Water Pollution Controls (NAC 445A - Water } \\
\text { Pollution Controls) }\end{array}$} \\
\hline $\begin{array}{l}\text { Value of 5-day Biological Oxygen Demand }\left(\mathrm{BOD}_{5}\right) \text {, total suspended } \\
\text { solids (TSS), and } \mathrm{pH} \text { in one sewage lagoon water sample sampled quarterly }\end{array}$ & $\begin{array}{l}\text { BOD5: varies } \\
\text { TSS: no limit } \\
\text { pH: } 6.0-9.0 \text { S.U. }\end{array}$ & $\begin{array}{l}\text { Compliant - Samples collected } \\
\text { in Jan., Apr., Jul, and Oct. }\end{array}$ & $\begin{array}{l}\text { 4.2.3.1; } \\
\text { Table 4-11 }\end{array}$ \\
\hline $\begin{array}{l}\text { Concentration of } 36 \text { contaminants in the filtrate from one sewage lagoon } \\
\text { sample collected annually from each of two permitted facilities }\end{array}$ & Limit varies & $\begin{array}{l}\text { Compliant - concentrations } \\
\text { within limits }\end{array}$ & $\begin{array}{l}4.23 .2 \\
\text { Table 4-12 }\end{array}$ \\
\hline
\end{tabular}




\begin{tabular}{|c|c|c|c|}
\hline Compliance Measure/Action & Compliance Limit & Compliance Status - 2004 & $\begin{array}{c}\text { Section } \\
\text { Reference }^{(\mathbf{a})}\end{array}$ \\
\hline $\begin{array}{l}\mathrm{pH} \text { value and concentration of } 18 \text { contaminants in a water sample collected } \\
\text { annually from groundwater monitoring well SM-23-1 }\end{array}$ & Limit varies & Compliant & $\begin{array}{l}4.23 .3 \\
\text { Table } 4-13\end{array}$ \\
\hline Inspection by operator of active sewage lagoon systems & Weekly & Compliant & 4.2 .3 .4 \\
\hline Inspection by operator of inactive sewage lagoon systems & Quarterly & Compliant & 4.2 .3 .4 \\
\hline $\begin{array}{l}\text { Submit quarterly monitoring reports for } 3 \text { active sewage lagoons (for Area } 6 \text {, } \\
12 \text {, and 23) }\end{array}$ & $\begin{array}{l}\text { Due end of Jan., Apr., } \\
\text { Jul., Oct. }\end{array}$ & Compliant & \\
\hline Allow NDEP access to conduct inspections of active sewage lagoon systems & 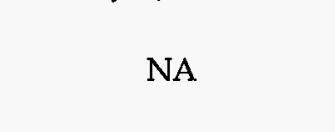 & $\begin{array}{l}\text { Compliant } \\
\text { Inspection conducted } \\
\text { April, } 2004\end{array}$ & 4.2 .3 .4 \\
\hline $\begin{array}{l}\text { Concentrations of tritium }\left({ }^{3} \mathrm{H}\right) \text {, gross alpha }(\alpha) \text {, and gross beta }(\beta) \text {, (in } \\
\text { picocuries per liter [pCi/L]), and } 16 \text { non-radiological contaminants/water } \\
\text { quality parameters in E Tunnel effluent water samples collected quarterly }\end{array}$ & $\begin{array}{c}{ }^{3} \mathrm{H}: 1,000,000 \mathrm{pCi} / \mathrm{L} \\
\alpha: 35.1 \mathrm{pCi} / \mathrm{L} \\
\beta: 101 \mathrm{pCi} / \mathrm{L} \\
\text { Non-rad: Limit varies }\end{array}$ & $\begin{array}{c}{ }^{3} \mathrm{H}: \quad 710,000 \mathrm{pCi} / \mathrm{L} \\
\alpha: 13.4 \mathrm{pCi} / \mathrm{L} \\
\beta: 72 \mathrm{pCi} / \mathrm{L} \\
\text { Non-rad: Compliant }\end{array}$ & $\begin{array}{l}\text { 4.1.6: } \\
\text { Table 4-6 }\end{array}$ \\
\hline $\begin{array}{l}\text { Concentrations of } 19 \text { contaminants in water samples from three NLVF sewage } \\
\text { outfalls and all sludge and liquid samples from the NLVF sand/oil interceptor }\end{array}$ & Limit varies & Compliant & $\begin{array}{l}\text { B.1.1.1; } \\
\text { Table B-2 }\end{array}$ \\
\hline $\begin{array}{l}\text { Concentrations of } 12 \text { contaminants in water samples from sewage outfall at } \\
\text { the RSL }\end{array}$ & Limit varies & Compliant & $\begin{array}{l}\text { B.3.1; } \\
\text { Table B-7 }\end{array}$ \\
\hline \multicolumn{4}{|l|}{$\begin{array}{l}\text { NAC } 534 \text { - Nevada Division of Water Resources Regulations for Water Well } \\
\text { and Related Drilling }\end{array}$} \\
\hline $\begin{array}{l}\text { Maintain state well-drilling license for personnel supervising well } \\
\text { construction/reconditioning }\end{array}$ & NA & $\begin{array}{l}\text { Compliant }-5 \text { licensed } \\
\text { personnel supervised well } \\
\text { activities }\end{array}$ & -- \\
\hline File notices of intent and affidavits of responsibility for plugging & NA & $\begin{array}{l}\text { Compliant - } 3 \text { notices of intent } \\
\text { with } 2 \text { affidavits were filed }\end{array}$ & -- \\
\hline Adhere to well construction requirements/waivers & NA & $\begin{array}{l}\text { Compliant - no new wells } \\
\text { constructed for UGTA Project; } \\
90 \text { boreholes plugged for } \\
\text { Borehole Management Program }\end{array}$ & -- \\
\hline Maintain required records and submit required reports & NA & Compliant & - - \\
\hline
\end{tabular}

(a) The section(s) within this document that describe how compliance summary data were collected

(b) Not detectable

(c) Compliance limit is specific for each contaminant; see referenced tables for specific limits

(d) Not applicable 


\subsection{Radiation Dose Protection}

Clean Air Act (CAA), National Emission Standards for Hazardous Air Pollutants (NESHAP) - NESHAP (40 CFR 61 Subpart $\mathrm{H})$ establishes a radiation dose limit of 10 millirem per year $(\mathrm{mrem} / \mathrm{yr})(0.1$ millisievert per year $[\mathrm{mSv} / \mathrm{yr}])$ to individuals in the general public from the air pathway. Sources of radioactive emissions on the NTS include: (1) evaporation of tritiated water (HTO) from containment ponds, (2) diffusion of HTO vapor from the soil at Area 5 Radioactive Waste Management Complex (RWMC), Sedan crater, and Schooner crater, (3) release of tritium gas during calibration of analytical equipment at Building 650 in Area 23, and (4) re-suspension of plutonium and americium from contaminated soil at nuclear device safety test and atmospheric test locations. NESHAP also specifies "Concentration Levels for Environmental Compliance" (abbreviated as CLs) for radionuclides. A CL is the annual average concentration of a radionuclide that could deliver a dose of $10 \mathrm{mrem} / \mathrm{yr}$. The CLs are provided for facilities which use air sampling at offsite receptor locations to demonstrate compliance.

Safe Drinking Water Act (SDWA) - The National Primary Drinking Water Regulations (40 CFR 141), promulgated by the SDWA (Federal Register, Vol. 65, No. 236, December 7, 2000), requires that the maximum contaminate level goal for any radionuclide be zero. But, when this is not possible (e.g., in groundwater containing naturally-occurring radionuclides), the SDWA specifies that the concentration of one or more radionuclides should not result in a whole body or organ dose greater than $4 \mathrm{mrem} / \mathrm{yr}(0.04 \mathrm{mSv} / \mathrm{yr})$. Sources of radionuclide contamination in groundwater are the numerous underground nuclear tests conducted at the NTS which were detonated near or below the water table.

DOE Order 450.1 Environmental Protection Program - This DOE Order requires federal facilities to: (1) conduct environmental monitoring to detect, characterize, and respond to releases from DOE activities, (2) assess impacts, (3) estimate dispersal patterns in the environment, (4) characterize the pathways of exposure to members of the public, (5) characterize the exposures and doses to individuals and to the population, and (6) evaluate the potential impacts to the biota in the vicinity of a DOE activity. Such releases, exposures, and doses apply to radiological contaminants.

DOE Order 5400.5 Radiation Protection of the Public and the Environment - Protection of the public and the environment is further mandated by this Order and by flow-down procedural standards established to help implement the objectives of the Order. DOE Order 5400.5 establishes requirements for: (1) measuring radioactivity in the environment, (2) applying the ALARA (As Low As Reasonably Achievable) process to all operations, (3) using mathematical models for estimating radiation doses, (4) releasing property having residual radioactive material, and (5) maintaining records demonstrating compliance with the requirements. DOE Order 5400.5 specifies a radiation dose limit of $100 \mathrm{mrem} / \mathrm{yr}(1 \mathrm{mSv} / \mathrm{yr})$ above background levels to individuals in the general public from all pathways of exposure combined. DOE Order 5400.5 also provides the derived concentration guides (DCGs) for all radionuclides. The DCGs are the annual average concentrations of a radionuclide that could deliver a dose of $100 \mathrm{mrem} / \mathrm{yr}$. The DCGs are provided as reference values to use in radiological protection programs at DOE facilities. The NESHAP CLs mentioned above are more conservative than one-tenth of the DCGs because they are computed with different dose models.

DOE Standard DOE-STD-1153-2002 - This Standard, titled A Graded Approach for Evaluating Radiation Doses to Aquatic and Terrestrial Biota (DOE, 2002a), provides methods, computer models, and guidance in implementing a graded approach to evaluating the radiation doses to populations of aquatic animals, terrestrial plants, and terrestrial animals residing on DOE facilities. A dose limit of $1 \mathrm{rad}$ per day ( $\mathrm{rad} / \mathrm{d})(10$ milligray per day [mGy/d]) for terrestrial plants and aquatic animals, and of $0.1 \mathrm{rad} / \mathrm{d}(1 \mathrm{mGy} / \mathrm{d})$ for terrestrial animals is specified by this DOE standard. Dose rates below these levels are believed to cause no measurable adverse effects to populations of plants and animals.

DOE Order 435.1 Radioactive Waste Management - This order ensures that all DOE radioactive waste is managed in a manner that is protective of the worker, public health and safety, and the environment. The directive manual for this Order (DOE M435.1-1) specifies that operations at the Area 3 and Area 5 Radioactive Waste Management Sites (RWMSs) must not contribute a dose to the general public in excess of $25 \mathrm{mrem} / \mathrm{yr}$. The order also directs how radioactive waste management operations are conducted on the NTS. These operational requirements are summarized in Section 2.4. 


\subsubsection{Compliance Reports}

In compliance with NESHAP under the CAA, the report National Emission Standards for Hazardous Air Pollutants, Calendar Year 2004, was submitted to EPA Region IX in June 2004. This Nevada Test Site Environmental Report 2004 was generated to report 2004 compliance with DOE Order 5400.5 and DOE-STD-1153-2002.

\subsubsection{Compliance Status}

Table 2-3 presents a summary of how NNSA/NSO complied with radiation protection regulations at the NTS and its satellite facilities in 2004 .

Table 2-3. NTS compliance status with regulations for radiation protection of the public and the environment

\begin{tabular}{|c|c|c|c|}
\hline Compliance Measure & Compliance Limit & $\begin{array}{l}\text { Compliance } \\
\text { Status - 2004 }\end{array}$ & $\begin{array}{c}\text { Section } \\
\text { Reference }^{(a)}\end{array}$ \\
\hline \multicolumn{4}{|l|}{ Clean Air Act - NESHAP } \\
\hline $\begin{array}{l}\text { Annual dose to the general public from all } \\
\text { radioactive air emissions }\end{array}$ & $\begin{array}{l}10 \mathrm{mrem} / \mathrm{yr} \\
(0.1 \mathrm{mSv} / \mathrm{yr})\end{array}$ & $\begin{array}{l}0.12 \mathrm{mrem} / \mathrm{yr} \\
(0.0012 \mathrm{mSv} / \mathrm{yr})\end{array}$ & $\begin{array}{l}3.1 .5 \\
8.1 .3\end{array}$ \\
\hline \multicolumn{4}{|l|}{ Safe Drinking Water Act } \\
\hline $\begin{array}{l}\text { Annual dose to the general public from drinking } \\
\text { water }\end{array}$ & $\begin{array}{c}4 \mathrm{mrem} / \mathrm{yr} \\
(0.04 \mathrm{mSv} / \mathrm{yr})\end{array}$ & $\begin{array}{l}0 \mathrm{mrem} / \mathrm{yr}^{(\mathrm{b})} \\
(0 \mathrm{mSv} / \mathrm{yr})\end{array}$ & $\begin{array}{l}\text { 4.1.4; } \\
\text { Table 4-1 }\end{array}$ \\
\hline \multicolumn{4}{|l|}{$\begin{array}{l}\text { DOE Order } 5400.5 \text { Radiation Protection of the Public } \\
\text { and the Environment }\end{array}$} \\
\hline $\begin{array}{l}\text { Annual dose above background levels to the general } \\
\text { public from all pathways }\end{array}$ & $\begin{array}{l}100 \mathrm{mrem} / \mathrm{yr} \\
(1 \mathrm{mSv} / \mathrm{yr})\end{array}$ & $\begin{array}{c}0.51 \mathrm{mrem} / \mathrm{yr} \\
(0.0051 \mathrm{mSv} / \mathrm{yr})\end{array}$ & $\begin{array}{l}\text { 8.1.6; Table 8-6; } \\
\text { B.1.5; Table } 8-5\end{array}$ \\
\hline $\begin{array}{l}\text { Total residual surface contamination of property } \\
\text { released offsite (in disintegrations per minute per } 100 \\
\text { square centimeters }\left[\mathrm{dpm} / 100 \mathrm{~cm}^{2}\right] \text { ) }\end{array}$ & $\begin{array}{l}300-15,000 \mathrm{dpm} / 100 \mathrm{~cm}^{2} \\
\text { depending on } \\
\text { radionuclide }\end{array}$ & $\begin{array}{l}\text { Compliant } \\
\text { No detectable } \\
\text { releases }\end{array}$ & 8.1 .5 \\
\hline \multicolumn{4}{|l|}{ DOE Standard 1153-2002 } \\
\hline Absorbed radiation dose to terrestrial plants & $\begin{array}{c}1 \mathrm{rad} / \mathrm{d} \\
(0.01 \mathrm{~Gy} / \mathrm{d})\end{array}$ & $\begin{array}{c}<1 \mathrm{rad} / \mathrm{d} \\
(<0.01 \mathrm{~Gy} / \mathrm{d})\end{array}$ & 8.2 \\
\hline Absorbed radiation dose to aquatic animals & $1 \mathrm{rad} / \mathrm{d}$ & $<1 \mathrm{rad} / \mathrm{d}$ & 8.2 \\
\hline Absorbed radiation dose to terrestrial animals & $\begin{array}{c}0.1 \mathrm{rad} / \mathrm{d} \\
(1 \mathrm{mGy} / \mathrm{d})\end{array}$ & $\begin{array}{l}<0.1 \mathrm{rad} / \mathrm{d} \\
(<1 \mathrm{mGy} / \mathrm{d})\end{array}$ & 8.2 \\
\hline \multicolumn{4}{|l|}{ DOE Order 435.1 Radioactive Waste Management } \\
\hline $\begin{array}{l}\text { Annual dose to the general public due to RWMS } \\
\text { operations }\end{array}$ & $\begin{array}{c}25 \mathrm{mrem} / \mathrm{yr} \\
(0.25 \mathrm{mSv} / \mathrm{yr})\end{array}$ & Compliant(c) & 5.3 .2 \\
\hline \multicolumn{4}{|l|}{ DOE Order 450.1 Environmental Protection Program } \\
\hline Conduct radiological environmental monitoring & $\mathrm{NA}^{(\mathrm{d})}$ & Compliant & $\begin{array}{l}3.1 ; 4.1 ; 5.0 \\
6.0 ; 7.0\end{array}$ \\
\hline Detect and characterize radiological releases & NA & Compliant & $\begin{array}{l}3.1 ; 4.1 ; 5.0 ; 6.0 \\
\text { Table } 3-12\end{array}$ \\
\hline Characterize pathways of exposure to the public & NA & Compliant & 8.1 .1 \\
\hline $\begin{array}{l}\text { Characterize exposures and doses to individuals, the } \\
\text { population, and biota }\end{array}$ & NA & Compliant & $\begin{array}{l}8.1 .6 ; 8.17 \\
8.2\end{array}$ \\
\hline
\end{tabular}

(a) The section(s) within this document that describe how compliance summary data were collected

(b) Migration of radioactivity in groundwater to offsite wells has never been detected

(c) Nearest populations to the Area 3 and 5 RWMSs are Amargosa Valley ( $55 \mathrm{~km}$ away) and Cactus Springs ( $36 \mathrm{~km}$ away), respectively. They are too distant to receive any radiation exposure from operations at the sites.

(d) Not applicable 


\subsection{Radioactive and Non-Radioactive Waste Management and Environmental Restoration}

10 CFR 830: Nuclear Safety Management - Establishes requirements for the safe management of DOE contractor and subcontractor work at DOE's nuclear facilities. It governs the possession and use of special nuclear material and byproduct materials deemed necessary for the protection of health and minimization of danger to life or property. Part 830 also covers activities at facilities where no nuclear material is present such as facilities that prepare the non-nuclear components of nuclear weapons, but which could cause radiological damage at a later time. It governs the conduct of the "management and operating contractor and other persons at DOE nuclear facilities" (including visitors to the facility). When coupled with the Price-Anderson Amendments Act (PAAA) of 1988 (Section 234A to the Atomic Energy Act), it provides DOE with authority to assess civil penalties for violation of rules, regulations or orders relating to nuclear safety by contractors, subcontractors, and suppliers who are indemnified under PAAA. The broad intent of the regulation is to ensure compliance with all enforceable rules, regulations, or orders relating to nuclear safety adopted by DOE for the NTS.

DOE Order 435.1 Radioactive Waste Management - Ensures that all DOE radioactive waste is managed in a manner that is protective of the worker, public health and safety, and the environment. Radioactive waste management activities conducted on the NTS which are subject to this Order include: (1) characterization of low level radioactive waste (LLW) and mixed low level radioactive waste (MW) generated by DOE within the state of Nevada, (2) disposal of LLW and MW at the RWMC which includes the Area 3 RWMS and the Area 5 RWMS,

(3) characterization, visual examination, and repackaging of transuranic (TRU) waste at the Waste Examination Facility (WEF) just south of the Area 5 RWMS, and (4) loading of TRU waste at the Mobile Loading Unit (MLU) at the Area 5 RWMS for shipment to the Waste Isolation Pilot Plant at Carlsbad, New Mexico.

Atomic Energy Act (AEA) of 1954 (42 U.S.C. Sect. 2011 et seq.) - Ensures the proper management of source, special nuclear, and byproduct material. At the NTS, AEA regulations are followed through compliance with DOE Order 435.1 and 10 CFR 830.

Resource Conservation and Recovery Act (RCRA) - Ensures the safe and environmentally responsible management of hazardous (see Glossary, Appendix D) and non-hazardous solid waste. RCRA $(1976,1996)$ and the Hazardous and Solid Waste Amendments of 1984 constitute the statutory basis for the regulation of hazardous waste and underground storage tanks (USTs). Under Section 3006, the EPA has authorized the state of Nevada to administer and enforce hazardous waste permits for many NNSA/NSO facilities. Nevada has issued a RCRA Hazardous Waste Operating Permit (NEV HW009) which governs operation of the Hazardous Waste Storage Unit (HWSU) in Area 5, the Explosive Ordnance Disposal Unit (EODU) in Area 11, and the disposal of MW at the Pit 3 Mixed Waste Disposal Unit (P03U) at the Area 5 RWMS. Under Subpart F of RCRA (40 CFR 265.92), groundwater monitoring is required to verify the performance of P03U. The NEV HW009 permit also prescribes post-closure monitoring for five closed waste sites on the NTS that are RCRA Part B-identified Corrective Action Units (CAUs). They include the Area 23 Hazardous Waste Trenches (CAU 112), the Area 3 U3fi Injection Well (CAU 91), the Area 3 U3ax/bl Subsidence Crater (CAU 110), the Area 2 Bitcutter Containment (CAU 90), and the Area 6 Decon Pond Facility (CAU 92).

The NTS has five USTs which are either (1) fully regulated under RCRA and registered with the state (1 tank), (2) regulated under RCRA and registered with the state, but deferred from leak detection requirements (1 tank), or (3) excluded from federal and state regulation ( 3 tanks). The NTS UST program reports, upgrades, and removes USTs in accordance with regulatory compliance schedules.

RCRA also requires generators of hazardous waste to have a program in place to reduce the volume or quantity and toxicity of such waste. These requirements and NTS compliance with them are addressed under the Pollution Prevention and Waste Minimization sections of this report (Section 2.7, Section 11.0).

The specific Nevada laws which govern hazardous waste management operations under Permit NEV HW009 are Disposal of Hazardous Waste (NRS 459-400 - 459.600), Facilities for Management of Hazardous Waste 
(NAC 444.842 - 444.8482), Disposal of Hazardous Waste (NAC 444.850 - 444.8746), and Limitations on Issuance of Permits (NAC 444.960).

Comprehensive Environmental Response, Compensation, and Liability Act (CERCLA)/ Superfund Amendments and Reauthorization Act (SARA) - Provides a framework for the cleanup of waste sites containing hazardous substances and an emergency response program in the event of a release of a hazardous substance to the environment. No hazardous waste cleanup operations on the NTS are regulated under CERCLA; they are regulated under RCRA instead. The only requirements of CERCLA applicable to NTS operations pertain to an emergency response program for hazardous substance releases to the environment (see discussion of Emergency Planning and Community Right-to-Know Act in Section 2.5).

Federal Facility Compliance Act (FFCA) - Extends the full range of enforcement authorities in federal, state, and local laws for management of hazardous wastes to federal facilities, including the NTS. The FFCA of 1992, signed by NNSA/NSO and the state of Nevada, requires identification of existing quantities for mixed waste, the proposal of methods and/or technologies of mixed waste treatment and management, the creation of enforceable timetables, and tracking and completion of deadlines.

Federal Facilities Agreement and Consent Order (FFACO) - Pursuant to Section 120(a)(4) of CERCLA and to Sections 6001 and 3004(u) of RCRA, the U.S. Department of Energy, U.S. Department of Defense, and the state of Nevada entered into a FFACO in May 1996. This FFACO addresses the environmental restoration of historically contaminated sites at the NTS, parts of Tonopah Test Range (TTR), parts of the Nevada Test and Training Range (NTTR) (formerly known as Nellis Air Force Range), the Central Nevada Test Area (CNTA), and the Project SHOAL Area. Under the FFACO, hundreds of historically contaminated sites on and off the NTS have been identified for cleanup and closure. Individual sites are called Corrective Action Sites (CASs). Multiple CASs are often grouped into CAUs.

40 CFR Subchapter I, Parts 239-299: Solid Wastes - At the NTS, these federal solid waste management regulations are followed through compliance with permits issued by the NDEP.

NAC 444.570-7499 - Solid Waste Disposal Controls - Enforces the federal regulations pertaining to solid wastes (40 CFR Subchapter I, Parts 239-299). This Nevada regulation sets standards for solid waste management systems, including the storage, collection, transportation, processing, recycling, and disposal of solid waste. The NTS has four permitted landfills for solid waste disposal which are regulated and permitted by the state: Area 5 Asbestiform Low-Level Solid Waste Disposal Site (P06U), Area 6 Hydrocarbon Disposal Site, Area 9 U10c Solid Waste Disposal Site, and Area 23 Solid Waste Disposal Site. These landfills are designed, constructed, operated, maintained, and monitored in adherence to the requirements of their state-issued permits.

\subsubsection{Compliance Reports}

The following reports were prepared in 2004 to comply with environmental regulations for waste management and environmental restoration operations on the NTS. All CAU or CAS reports prepared in 2004 as per the FFACO schedule for environmental restoration of contaminated sites are presented in Table 9.4 of Section 9.4 .1 .

- Annual Asbestos Disposal Report (for the Area 5 Asbestiform Low-Level Solid Waste Disposal Site P06U)

- Quarterly $L L W / M L L W$ Disposal Reports (for all active LLW and MW disposal cells)

- Biannual Neutron Monitoring Report for the Nevada Test Site Area $910 \mathrm{c}$ and Area 6 Hydrocarbon Landfills

- Nevada Test Site 2004 Data Report: Groundwater Monitoring Program Area 5 Radioactive Waste Management Site

- Post-closure monitoring reports for the five RCRA Part B-identified CAUs

- January-June 2004 Biannual Solid Waste Disposal Site Report for the Nevada Test Site Area 23 Sanitary Landfill

- July-December 2004 Biannual Solid Waste Disposal Site Report for the Nevada Test Site Area 23 Sanitary Landfill 
- 2004 Annual Solid Waste Disposal Site Report for the Nevada Test Site Area 6 Hydrocarbon Landfill and Area 9 U10c Landfills

\subsubsection{Compliance Status}

See Table 2-4 for a summary of how NNSA/NSO complied with waste management and environmental restoration regulations at the NTS in 2004. 
Table 2-4. NTS compliance status with applicable waste management and environmental restoration regulations

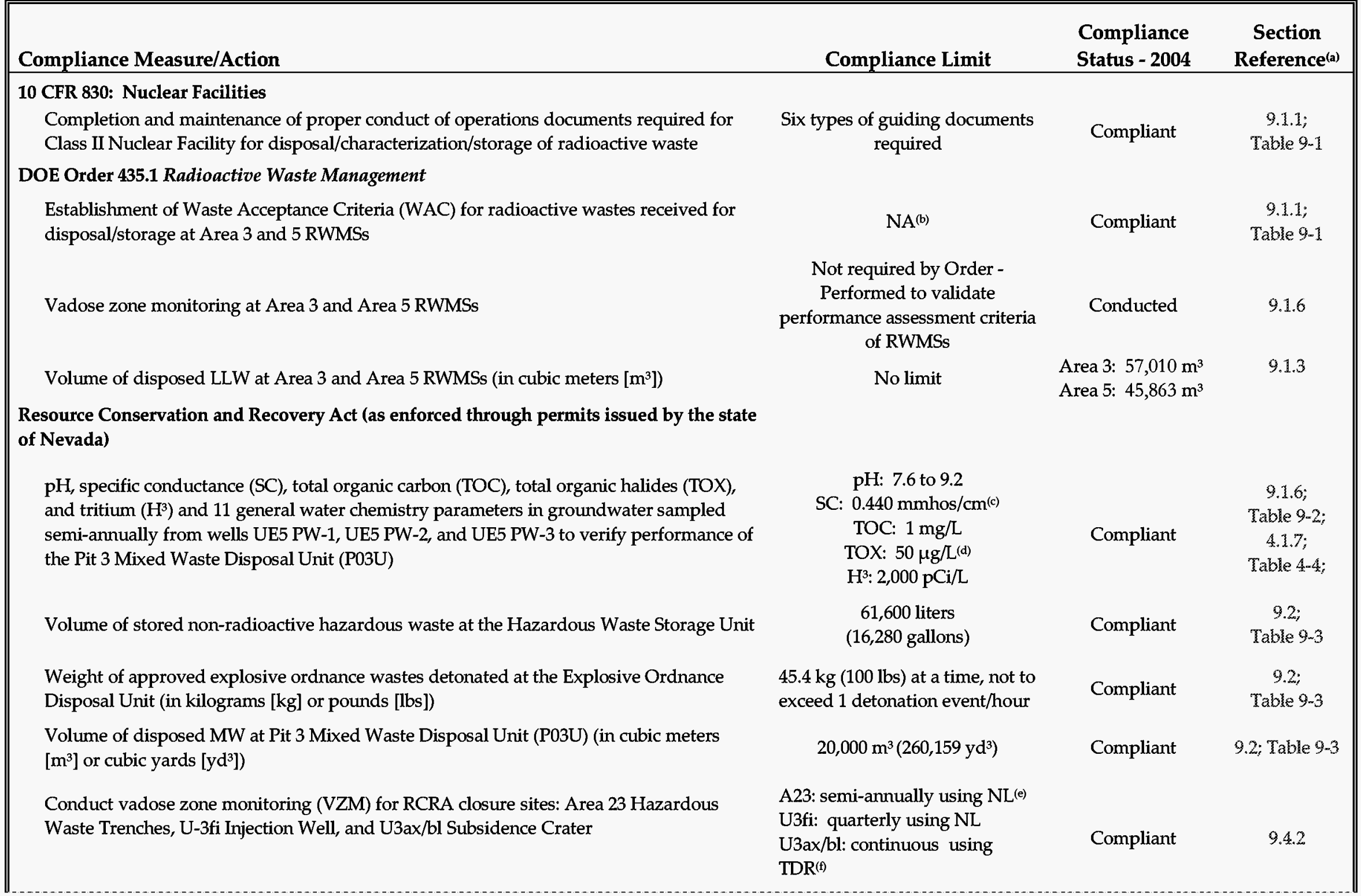


Table 2-4. (continued)

\begin{tabular}{||l}
\hline Compliance Measure/Action \\
\hline Periodic post-closure inspection of Area 2 Bitcutter Containment and Area 6 Decon Pond
\end{tabular}

Upgrade, remove, and report on underground storage tanks (USTs)

\section{Federal Facilities Agreement and Consent Order}

Adherence to calendar year work scope for site characterization, remediation, and closures

Post-closure monitoring and inspections of closed sites

\section{NAC 444.750-8396 - Solid Waste Disposal Controls}

Track weight and volume of waste disposed each calendar year

Monitor vadose zone for the Area 6 Hydrocarbon and Area 9 U10c Solid Waste disposal sites

Monitor groundwater quality at Well SM-23-1 for the Area 23 Solid Waste Disposal Site

Compliance Limit

Compliance

NA

NA

33 CAUs identified for some phase of action; 56 CASs were closed

23 Sites required monitoring/ inspecting

Area 5 P06U - No limit

Area 6 - No limit

Area 9 - No limit

Area 23 - 20 tons/d

Annually using NL(e)

In June 2004, NDEP granted a groundwater monitoring exclusion
Section

Status - 2004

Compliant

Compliant

Compliant

All milestones

were met

Compliant eference $^{(a)}$

9.4 .2

9.3

9.4

Table $9-4$

Compliant

9.5

Table 9.5

Compliant

NA

(a) The section(s) within this document that describe how compliance summary data were collected

(b) Not applicable

(c) $\mathrm{mmhos} / \mathrm{cm}=$ milli-mhos per centimeter

(d) $\mu \mathrm{g} / \mathrm{L}=$ micrograms per liter

(e) Neutron logging through access tubes

(f) Time domain reflectometry sensors 


\subsection{Hazardous Materials Control and Management}

Toxic Substances Control Act (TSCA) - Requires testing and regulation of chemical substances that enter the consumer market. Since the NTS does not produce chemicals, compliance with TSCA is primarily directed toward management of polychlorinated biphenyls (PCBs). The regulations implementing TSCA for the state of Nevada contain record keeping requirements for PCB activities (NAC 444.9452). At the NTS, remediation activities and maintenance of fluorescent lights can result in the disposal of PCB-contaminated waste and light ballasts. Disposal of these items on the NTS are regulated.

Federal Insecticide, Fungicide, and Rodenticide Act (FIFRA) - Sets forth procedures and requirements for pesticide registration, labeling, classification, devices for use, and certification of applicators. The use of certain pesticides (called "restricted-use pesticides") are regulated. The use of non-restricted-use pesticides (as available in consumer products) is not regulated. On the NTS, both restricted-use and non-restricted-use pesticides are applied under the direction of a state of Nevada certified applicator. Pesticide applications in food service facilities are subcontracted to state-certified vendors who provide these services.

Emergency Planning and Community Right-to-Know Act (EPCRA) - This act is a free-standing provision under Title III of the 1986 Superfund Amendments and Reauthorization Act (SARA Title III) amendments to CERCLA. It requires that federal, state, and local emergency planning authorities be provided information regarding the presence and storage of hazardous substances and their planned and unplanned environmental releases, including provisions and plans for responding to emergency situations involving hazardous materials. EO 13148 Federal Compliance with Right-to-Know Laws and Pollution Prevention Requirements, requires all federal facilities to comply with the provisions of EPCRA. Under EPCRA, NNSA/NSO is required to submit reports pursuant to Sections 302, 304, 311,312 , and 313 of SARA Title III described below.

Section 302-303, Planning Notification - Requires that the state emergency response commission and the local emergency planning committee be notified when an extremely hazardous substance (EHS) is present at a facility in excess of the threshold planning quantity. An inventory of the location and amounts of all hazardous substances stored on the NTS and its satellite facilities is maintained. Inventory data are included in an annual report called the Nevada Combined Agency (NCA) Report. Also, NNSA/NSO monitors hazardous materials while they are in transit on the NTS through a hazardous materials notification system called HAZTRAK®.

Section 304, Extremely Hazardous Substances Release Notification - Requires that the local emergency planning committee and state emergency response agencies be notified immediately of accidental or unplanned releases of an EHS to the environment. Also, the national response center is notified if the release exceeds the CERCLA reportable quantity for the particular hazardous substance.

Section 311-312, Material Safety Data Sheet (MSDS)/Chemical Inventory - Requires facilities to provide applicable emergency response agencies with MSDSs, or a list of MSDSs for each hazardous chemical stored on site. This is essentially a one-time reporting unless chemicals or products change. Any new MSDSs are provided annually in the NCA Report. Section 312 requires facilities to report maximum amounts of chemicals onsite at any one time. This report is submitted to the State Emergency Response Commission, the Local Emergency Planning Committee, and the local fire departments.

Section 313, Toxic Release Inventory (TRI) Reporting - Requires facilities to submit an annual report entitled "Toxic Chemical Release Inventory, Form R" to the EPA and to the state if annual usage quantities of listed toxic chemicals exceed specified thresholds. Lead releases on the NTS above threshold limits are reported to the EPA and the State Emergency Response Commission in the TRI, Form R report.

NAC 555 - Control of Insects, Pests, and Noxious Weeds - Provides regulatory framework for certification of several classifications of registered pesticide and herbicide applicators in the state of Nevada. The Nevada 
Department of Agriculture (NDOA) administers this program and has the primary role to enforce FIFRA in Nevada. Inspections of pesticide/herbicide applicator programs are carried out by NDOA. Restricted-use pesticides are not used by BN at the NTS.

NAC 444 - Polychlorinated Biphenyls - This code incorporates by reference the federal requirements for the handling, storage, and disposal of PCBs at the NTS.

State of Nevada Chemical Catastrophe Prevention Act - This state act directed the NDEP to develop and implement an accident prevention program which was named the Chemical Accident Prevention Program (CAPP). The act requires registration of facilities storing EHSs above listed thresholds. A report is submitted to the NDEP if any storage quantity thresholds are exceeded.

\subsubsection{Compliance Reports}

The following reports were generated for NNSA/NSO operations in 2004 on the NTS and its satellite facilities in compliance with hazardous materials control and management regulations:

- Nevada Combined Agency Report - Calendar Year 2004, submitted to state and local agencies on February 25, 2005

- Toxic Release Inventory Report, Form R for CY2004 Operations, submitted to the EPA and to the state on June 22, 2005

- Calendar Year (CY) 2003 Polycblorinated Biphenyls (PCBs) Report for the Nevada Test Site (NTS), submitted to NNSA/NSO on March 22, 2004. (This report is no longer required to be submitted to the EPA).

- Calendar Year (CY) 2004 Polycblorinated Biphenyls (PCBs) Report for the Nevada Test Site (NTS), submitted to NNSA/NSO on March 22, 2005. (This report is no longer required to be submitted to the EPA).

\subsubsection{Compliance Status}

See Table 2-5 for a summary of how NNSO/NSA complied with regulations for hazardous materials control and management at the NTS and its satellite facilities in 2004. 
Table 2-5. NTS compliance status with applicable regulations for hazardous substance control and management

\begin{tabular}{|c|c|c|c|}
\hline Compliance Measure/Action & Compliance Limit & Compliance Status - 2004 & $\begin{array}{c}\text { Section } \\
\text { Reference }^{(\mathrm{a})}\end{array}$ \\
\hline \multicolumn{4}{|l|}{$\begin{array}{l}\text { Toxic Substances Control Act (TSCA) and } \\
\text { NAC } 444 \text { - Polychlorinated Biphenyls }\end{array}$} \\
\hline Storage and offsite disposal of PCB materials & Required if $>50 \mathrm{ppm}^{(\mathrm{b})} \mathrm{PCBs}$ & Compliant & 10.1 \\
\hline Storage and onsite disposal of $\mathrm{PCB}$ materials & Allowed if $<50 \mathrm{ppm}$ PCBs & Compliant & 10.1 \\
\hline $\begin{array}{l}\text { Disposal of bulk product waste (BPW) containing PCBs } \\
\text { generated by remediation and site operations }\end{array}$ & $\begin{array}{l}\text { Case-by-case approval by } \\
\text { NDEP }\end{array}$ & Compliant & 10.1 \\
\hline $\begin{array}{l}\text { Generate report of quantities of PCB liquids and materials } \\
\text { disposed offsite during previous calendar year }\end{array}$ & Due July 1 of following year & $\begin{array}{l}\text { Compliant - } \\
\text { submitted March 22, } 2004 \text { for CY 2003; } \\
\text { submitted March 22, } 2005 \text { for CY 2004 }\end{array}$ & 10.1 \\
\hline \multicolumn{4}{|l|}{$\begin{array}{l}\text { Federal Insecticide, Fungicide, and Rodenticide Act (FIFRA) and } \\
\text { NAC } 555 \text { - Control of Insects, Pests, and Noxious Weeds }\end{array}$} \\
\hline $\begin{array}{l}\text { Application of restricted-use pesticides are conducted under } \\
\text { the direct supervision of a state-certified applicator }\end{array}$ & $\mathrm{NA}^{(\mathrm{c})}$ & $\begin{array}{l}\text { Compliant - } \\
\text { no restricted-use pesticides were applied }\end{array}$ & 10.2 \\
\hline $\begin{array}{l}\text { Maintain state certification of onsite pesticide and herbicide } \\
\text { applicator }\end{array}$ & NA & Compliant & 10.2 \\
\hline \multicolumn{4}{|l|}{ Emergency Planning and Community Right-to-Know Act (EPCRA) } \\
\hline Section 302-303 Planning Notification & NCA Report due in March & $\begin{array}{l}\text { Compliant - } \\
\text { submitted February 25, 2005; } \\
\text { no EHS thresholds exceeded }\end{array}$ & $\begin{array}{l}\text { 10.3; B.1.4; } \\
\text { B.3.3 }\end{array}$ \\
\hline Section 304 - EHS Release Notification & $\begin{array}{l}\text { Notification Report due } \\
\text { immediately after a release }\end{array}$ & $\begin{array}{l}\text { Compliant - } \\
\text { no releases occurred }\end{array}$ & $\begin{array}{l}\text { 10.3; B.1.4; } \\
\text { B.3.3 }\end{array}$ \\
\hline Section 311-312-MSDS/Chemical Inventory & NCA Report due in March & $\begin{array}{l}\text { Compliant - } \\
\text { submitted February 25, } 2005\end{array}$ & $\begin{array}{l}\text { 10.3; B.1.4; } \\
\text { B.3.3 }\end{array}$ \\
\hline Section 313 - TRI Reporting & TRI Report, Form R due July 1 & $\begin{array}{l}\text { Compliant - } \\
\text { submitted June 22, } 2005 \text { - lead was the } \\
\text { only reportable substance }\end{array}$ & $\begin{array}{l}\text { 10.3; B.1.4; } \\
\quad \text { B.3.3 }\end{array}$ \\
\hline \multicolumn{4}{|l|}{ State of Nevada Chemical Catastrophe Prevention Act } \\
\hline $\begin{array}{l}\text { Registration of NTS with the state if EHSs are stored above } \\
\text { listed threshold quantities }\end{array}$ & $\begin{array}{l}\text { NDEP-CAPP(d) Report due } \\
\text { June 21, } 2005\end{array}$ & $\begin{array}{l}\text { Compliant - no threshold quantities } \\
\text { exceeded, no report submitted }\end{array}$ & 10.4 \\
\hline
\end{tabular}

(a) The section(s) within this document that describe how compliance summary data were collected

(b) $\mathrm{ppm}=$ parts per million

(c) Not applicable

(d) Chemical Accident Prevention Program 